WHC-SP-0098-6

Revision 0

UC-600

\title{
Routine Environmental Monitoring Schedule, Calendar Year 1995
}

\author{
J. W. Schmidt \\ B. M. Markes \\ S. M. McKinney
}

Date Published

December 1994

Prepared for the U.S. Department of Energy Office of Environmental Restoration and Waste Management

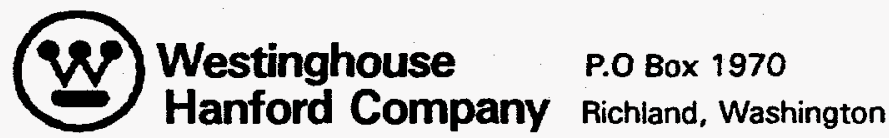

Hanford Operations and Engineering Contractor for the

U.S. Department of Energy under Contract DE-AC06-87RL10930 


\section{DISCLAIMER}

This document was prepared as an account of work sponsored by an agency of the United States Government. Neither the United States Government nor the University of California nor any of their employees, makes any warranty, express or implied, or assumes any legal liability or responsibility for the accuracy, completeness, or usefulness of any information, apparatus, product, or process disclosed, or represents that its use would not infringe privately owned rights. Reference herein to any specific commercial products, process, or service by trade name, trademark, manufacturer, or otherwise, does not necessarily constitute or imply its endorsement, recommendation, or favoring by the United States Government or the University of California. The views and opinions of authors expressed herein do not necessarily state or reflect those of the United States Government or the University of California, and shall not be used for advertising or product endorsement purposes. 


\section{DISCLAIMER}

Portions of this document may be illegible in electronic image products. Images are produced from the best available original document. 
ROUTINE ENVIRONMENTAL MONITORING SCHEDULE,

Prepared By:
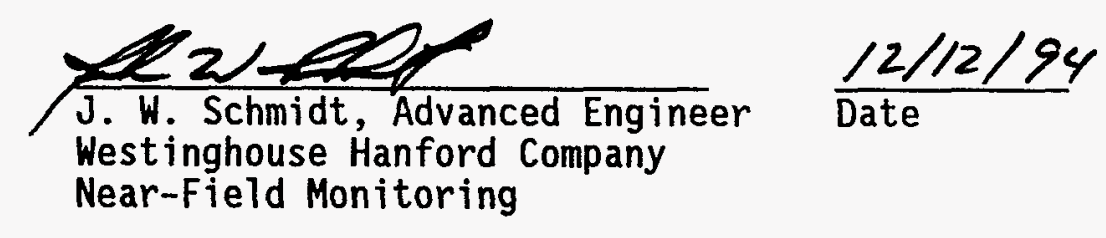

Reviewed/Approved By:
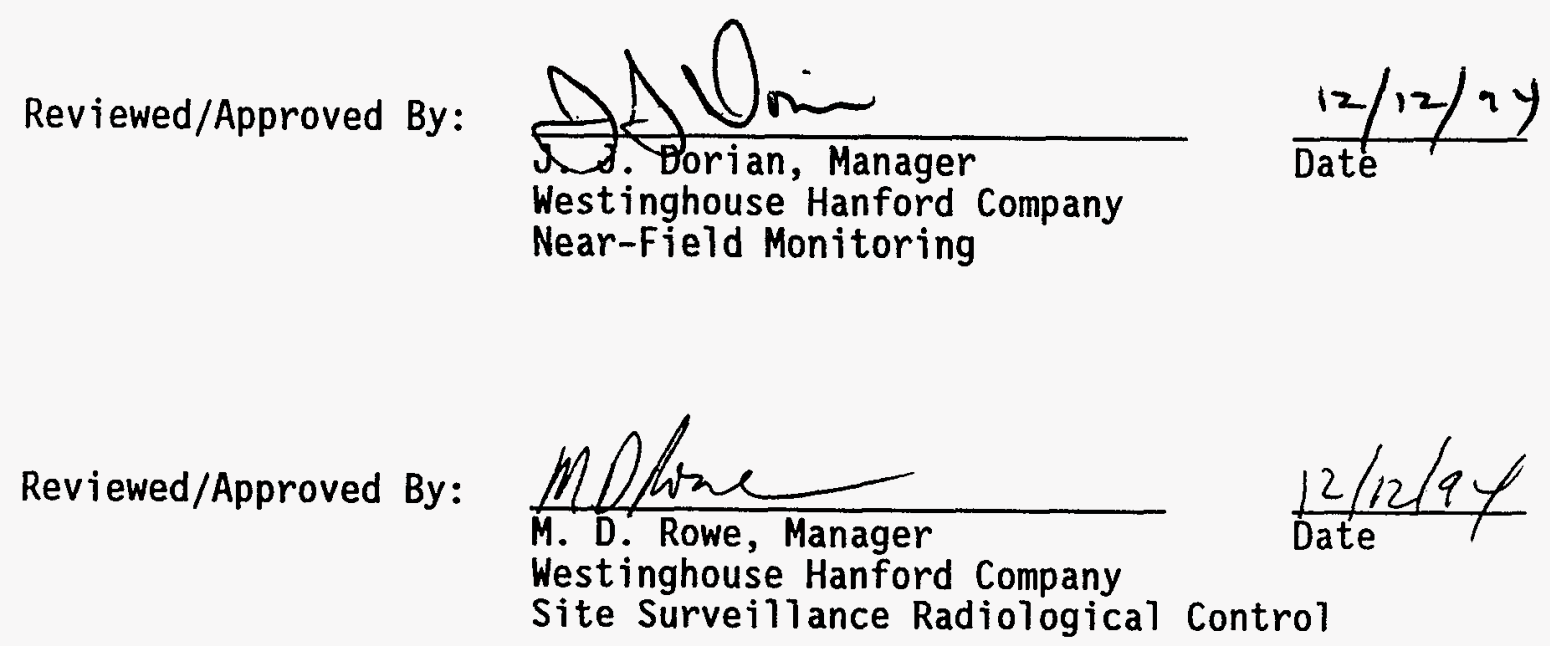

Reviewed/Approved By:

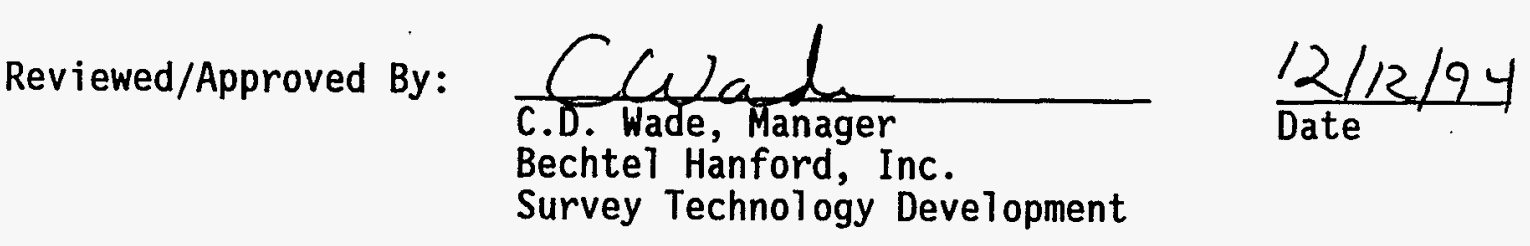

$\frac{12 / 12 / 94}{\text { Date }}$

Bechtel Hanford, Inc. Westinghouse Hanford Company

Site Surveillance Radiological Control 


\section{RELEASE AUTHORIZATION}

Document Number: WHC-SP-0098-6

Document Title: $\quad$ Routine Environmental Monitoring Schedule, Calendar

Year 1995

Release Date: $\quad 12 / 13 / 94$

This document was reviewed following the
procedures described in WHC-CM-3-4 and is:

APPROVED FOR PUBLIC RELEASE

WHC Information Release Administration Specialist:

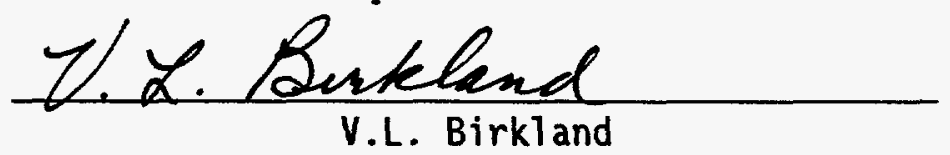

$12 / 13 / 94$

TRADEMARK DISCLAIMER. Reference herein to any specific commercial product, process, or service by trade name, trademark, manufacturer, or otherwise, does not necessarily constitute or imply its endorsenent, recommendation, or favoring by the United States Government or any agency thereof or its contractors or subcontractors.

This report has been reproduced from the best available copy. Available in paper copy and microfiche. Printed in the United States of America. Available to the U.S. Department of Energy and its contractors from:

U.S. Department of Energy

Office of Scientific and Technical Information (OSTI)

P.0. Box 62

Oak Ridge, TN 37831

Telephone: (615) 576-8401

Available to the public from:

U.S. Department of Conmerce

National Technical Information Service (NTIS)

5285 Port Royal Road

Springfield, VA 22161

Telephone: (703) 487-4650 
WHC-SP-0098-6

CONTENTS

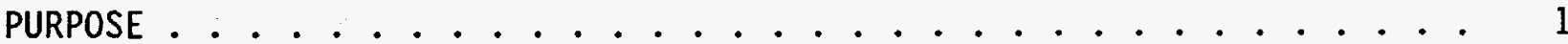

ENVIRONMENTAL RESTORATION CONTRACTOR

(BECHTEL HANFORD, INC.) ANNUAL SCHEDULE .............. 3

JANUARY/FEBRUARY/MARCH .............. 5

APRIL/MAY/JUNE ................... . . . . 6

JULY/AUGUST/SEPTEMBER . . . . . . . . . . . . . 8

OCTOBER/NOVEMBER/DECEMBER . . . . . . . . . . . . . 9

OPERATIONS AND MAINTENANCE CONTRACTOR

(WESTINGHOUSE HANFORD COMPANY) ANNUAL SCHEDULE . . . . . . . . . . . 11

JANUARY $\ldots \ldots . \ldots 13$

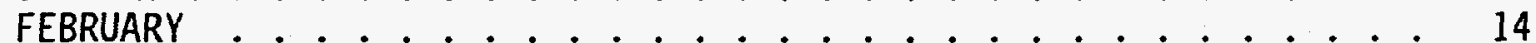

MARCH . . . . . . . . . . . . . 15

APRIL . . . . . . . . . . . . . . 16

MAY . . . . . . . . . . . . . . . 17

JUNE . . . . . . . . . . . . . . . . 18

JULY . . . . . . . . . . . . . . . . . . . 19

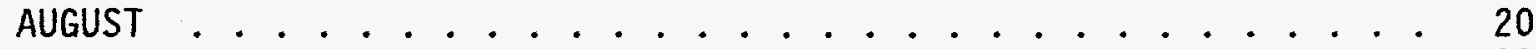

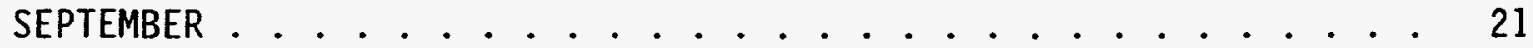

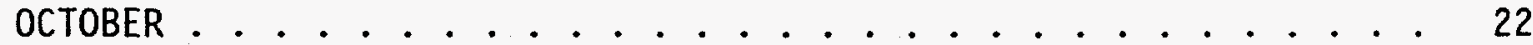

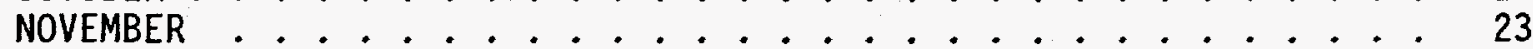

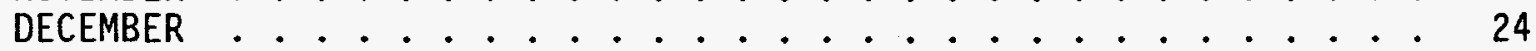

APPENDIX A SURVEY SITES AND SAMPLE LOCATIONS

APPENDIX B DESCRIPTIONS OF 200 AREA UNPLANNED RELEASE SITES 
WHC-SP-0098-6

\section{TERMS}

$\begin{array}{ll}\text { BHI } & \text { Bechtel Hanford, Inc. } \\ \text { DSI } & \text { (internal memorandum) } \\ \text { LERF } & \text { liquid effluent retention facility } \\ \text { OEM } & \text { Operational Environmental Monitoring } \\ \text { PUREX } & \text { Plutonium-Uranium Extraction (Plant) } \\ \text { RC } & \text { Radiological Control } \\ \text { REDOX } & \text { Reduction-0xidation (S Plant) } \\ \text { TLD } & \text { Thermoluminescent dosimeter } \\ \text { URM } & \text { underground radiation material } \\ \text { WHC } & \text { Westinghouse Hanford Company }\end{array}$




\section{PURPOSE}

This document provides Bechtel Hanford, Inc. (BHI) and Westinghouse Hanford Company (WHC) a schedule in accordance with the WHC-CM-7-5, Environmenta 7 Compliance, of monitoring and sampling routines for the Operational Environmental Monitoring (OEM) program during calendar year (CY) 1995. Every attempt will be made to consistently follow this schedule; any deviation from this schedule will be documented by an internal memorandum (DSI) explaining the reason for the deviation. The DSI will be issued by the scheduled performing organization and directed to Near-Field Monitoring.

The survey frequencies for particular sites are determined by the technical judgment of Near-Field Monitoring and may depend on the site history, radiological status, use, and general conditions. Additional surveys may be requested at irregular frequencies if conditions warrant. All radioactive wastes sites are scheduled to be surveyed at least annually. Any newly discovered wastes sites not documented by this schedule will be included in the revised schedule for CY 1995.

This schedule does not discuss staffing needs, nor does it list the monitoring equipment to be used in completing specific routines. Personnel performing routines to meet this schedule shall communicate any need for assistance in completing these routines to Radiological Control management and Near-Field Monitoring.

After each routine survey is completed, a copy of the survey record, maps, and data sheets will be forwarded to Near-Field Monitoring. These routine surveys will not be considered complete until this documentation is received. At the end of each month, Radiological Control (RC) shall forward a copy of the Routine Sign-off Sheet and a DSI validating the completion of the scheduled routine surveys for that month.

${ }^{1}$ WHC-CM-7-5, Environmental Compliance, Westinghouse Hanford Company, Richland Washington. 
WHC-SP-0098-6

This page intentionally left blank. 
WHC-SP-0098-6

\section{ENVIRONMENTAL RESTORATION CONTRACTOR}

(BECHTEL HANFORD, INC.)

ANNUAL SCHEDULE 


\section{WHC-SP-0098-6}

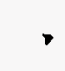

This page intentionally left blank. 
WHC-SP-0098-6

\section{JANUARY/FEBRUARY/MARCH}

QUARTERLY (Complete in March)

1. Waste site visual survey (posting, housekeeping, vegetation control, animal activity, unusual conditions, etc.).

\section{ANNUALLY}

1. $316-1,316-2,316-3,316-4$, and 316-5.

2. 618-1, 618-2, 618-3, 618-4, 618-5, 618-7, 618-8, 614-4, 618-10, 618-11.

3. 216-N-2 through $216-\mathrm{N}-7$.

4. 216-A-25 covered pond.

5. 216-B-3-1, 216-B-3-2, and 216-B-3-3 covered ditches (includes UN-216-E-40).

6. 216-B-3 stabilized pond.

7. 216-B-3A.

8. $216-B-3 B$.

9. 216-A-29 ditch.

10. $216-A-24$.

11. UN-216-E-33.

12. $216-A-18$.

13. $216-\mathrm{A}-19$ and $216-\mathrm{A}-20$.

14. 216-A-37-1.

15. $216-A-6$.

16. 216-A-5 (inside the Plutonium-Uranium Extraction (PUREX) Plant exclusion fence).

17. 200 East $B$ cribs and trenches, drains, and reverse wells (216-B-5, 216-B-6, 216-B-7A, 216-B-7B, 216-B-8, 216-B-9, 241-B-361 tank, 216-B-11A, 216-B-11B, 216-B-12, 216-B-51, 216-B-56, 216-B-57, and 216-B-43 through 216-B-50).

18. 216-B-10A and 216-B-10B.

19. 200-E $C$ cribs and trenches $(216-C-1,216-C-3,216-C-5,216-C-6,216-C-8$, $216-(-10)$.

20. $218-C-9$.

21. 216-B-2-1, 216-B-2-2, and 216-B-2-3 covered ditches.

22. 216-B-35 through 216-B-42 trenches.

23. $218-\mathrm{E}-1$.

24. $218-E-2 A$.

25. $218-\mathrm{E}-4$.

26. 218-E-12A.

27. 218-E-12B stabilized portion (includes UN-216-E-32).

28. $218-\mathrm{E}-8$.

29. $216-A-10$ crib.

30. Cribs and trenches, drains, and reverse wells, 216-A-1, 216-A-3, $216-A-7,216-A-9,216-A-27,216-A-36 A$ and $-36 B$.

31. UN-216-E-38. 
WHC-SP-0098-6

\section{APRIL/MAY/JUNE}

QUARTERLY (Complete in June)

1. Waste site visual survey (posting, housekeeping, vegetation control, animal activity, unusual conditions, etc.).

\section{SEMI-ANNUALLY (Complete in May)}

1. BC cribs, controlled area roads, and firebreaks (UN-216-E-11).

\section{ANNUALLY}

1. UN-216-E-17.

2. $216-A-34$.

3. 218-E-2, 218-E-5A, 218-E-5B, and 218-E-9 burial grounds.

4. 218-E-10 burial ground (stabilized portion).

5. $\mathrm{BC}$ cribs, 216-B-14 through 216-B-19.

6. $B C$ trenches, 216-B-20 through 216-B-34, 216-B-52, 216-B-53A and 216-B-53B, 216-B-54, 216-B-58.

7. 218-E-7 burial vault.

8. 216-S-17.

9. $216-S-5,216-S-6$.

10. 216-S-171, 216-S-172.

11. 216-U-11.

12. $216-\mathrm{S}-3$ and $216-\mathrm{S}-15$.

13. 216-S-16.

14. 216-S-10 ditch.

15. 216-S-19.

16. 216-S cribs and trenches (216-S-1, 216-S-2, 216-S-4, 216-S-5, 2904-S-170, and 2904-S-160 weirs, 216-S-7, 216-S-8, 216-S-9, 216-S-12, $216-S-13,216-S-20,216-S-21,216-S-22,216-S-23)$.

17. 216-U cribs and trenches $(216-U-1,216-U-2,216-U-3,216-U-5,216-U-6$, 216-U-8) and 241-U-361 tank.

18. 218-W-1, 218-W-2, 218-W-4A, 218-W-4B (stabilized part), and 218-W-11. 19. $216-Z-4,216-Z-5,216-Z-6,216-Z-7,216-Z-10$, and $216-Z-17$ stabilized areas.

20. 216-U-14 ditch (inactive section from 19th Street to 207-U Basin, from 207-U to U Pond).

21. 216-T cribs, trenches, and reverse wells 216-T-2, 216-T-3, and 241-T-361 catch tank, 216-T-5 tile field(to include 216-T-7), 216-T-6, 216-T-8, 216-T-18, 216-T-19, 216-T-20, 216-T-26 through 216-T-28, 216-T-33, $216-\mathrm{T}-34,216-\mathrm{T}-35$, and $216-\mathrm{T}-36$.

22. 204-S stabilized area.

23. 216-T-14 through 216-T-17.

24. 216-U-10 covered pond (includes UN-216-W-14, UN-216-W-15, UN-216-W-16, and UN-216-W-17).

25. UN-216-W-23.

26. 218-W-1A burial ground.

27. 218-W-2A burial ground.

28. 218-W-3 burial ground.

29. 216-T-21 through 216-T-25 trenches. 
30. UN-216-W-7.

31. UN 216-W-5.

32. $218-W-7$ burial vault, $218-W-8$ burial vault.

33. $218-W-9$ burial vault (includes UN-216-W-19).

34. $216-Z$ cribs and trenches $(216-Z-7,216-Z-8,216-Z-11,216-Z-12,216-Z-16$, $216-Z-18,216-Z-19,216-Z-20$ (includes UN-216-W-20)).

35. 207-S retention basin (includes UN-216-W-2).

36. UN-216-W-38.

37. Reduction-0xidation (S Plant) (REDOX) railroad spur.

38. 216-U-12.

39. 216-T-4-2 (216-T-4A) pond.

40. T Plant waste unloading station (alpha contamination).

41. Unplanned release site surveys 200-W: UN-216-W-24, UN-216-W-25, UN-216-W-26, UN-216-W-29, UN-216-W-30, and UN-216-W-31.

42. 216-U-4 reverse wel1.

43. 216-U-4A French drain.

44. 216-U-4B reverse well.

45. 216-U-7 French drain.

46. 216-U-15 trench. 
WHC-SP-0098-6

\section{JULY/AUGUST/SEPTENBER}

\section{QUARTERLY (Complete in September)}

1. Waste site visual survey (posting, housekeeping, vegetation control, animal activity, unusual conditions, etc.).

\section{ANNUALLY}

1. 100-F underground radiation material (URM) area [includes: 116-F-2, $116-F-5,116-F-6,116-F-8,116-F-9,116-F-10,116-F-12,116-F-13$, 118-F-3, UN-100-F-1 (UPR-100-F-1)].

2. 105-F exclusion area (includes: 116-F-3, 116-F-4, 116-F-11, 118-F-4).

3. 107-F retention basin.

4. 100-F ash pit.

5. 116-F-1 Lewis canal.

6. 118-F-1 burial ground.

7. 118-F-2 burial ground.

8. 118-F-5 burial ground.

9. 118-F-6 burial ground.

10. 116-F-7 crib (117-F-7) 68 meters due south of exclusion fence.

11. 100-H URM area [includes: $116-\mathrm{H}-1,116-\mathrm{H}-2,116-\mathrm{H}-5,116-\mathrm{H}-4$ (105-H Pluto (rib)].

12. 105-H exclusion area (includes: $116-\mathrm{H}-3,118-\mathrm{H}-4,118-\mathrm{H}-5$ ).

13. 116-H-6 solar basin (183-H).

14. 116- $\mathrm{H}-7$ retention basin.

15. 118- $\mathrm{H}-1$ burial ground.

16. 118- $\mathrm{H}-2$ burial ground.

17. 118- $\mathrm{H}-3$ burial ground.

18. 116-H-9 (117-H crib) 28 meters west/27 meters north of the southwest corner of 105-H exclusion fence.

19. 100 D/DR URM area (includes: 116-DR-1 and 116-DR-2 (107 trenches), 116-DR-5, 116-DR-6, 118-D-3, 118-D-4, 116-D-5.

21. $105 \mathrm{D} / \mathrm{DR}$ exclusion area (includes: 116-D-1A, 116-D-1B, 116-D-2, 116-D-3, $116-D-4,116-D-6,116-D R-7,116-D R-3,116-D R-4)$.

22. 118-D-1 burial ground.

23. 118-D-2 burial ground.

24. 116-D-9 (117-D crib).

25. 118-D-5 burial ground.

26. 118-DR-1 burial ground.

27. 116-DR-8.

28. 100-D/DR contamination area (includes 116-D-7, 116-DR-9, and pipeline.

29. D island vent risers and surrounding area. 
WHC-SP-0098-6

\section{OCTOBER/NOVEMBER/DECEMBER}

\section{QUARTERLY (Complete in December)}

1. Waste site visual survey (posting, housekeeping, vegetation control, animal activity, unusual conditions, etc.).

\section{SEMI-ANNUALLY}

1. BC cribs, controlled area roads, and firebreaks (UN-216-E-11).

\section{ANNUALLY}

1. 100-N Area micro-R survey (1301-N (116-N-1), 1325-N (116-N-3), N Springs (to be completed in October).

2. 1325-N (116-N-3) fence line.

3. $1301-\mathrm{N}(116-\mathrm{N}-1)$ fence line.

4. 116-N-4 [emergency dump basin [1iquid effluent retention facility (LERF)] \} fence line.

5. 118-N-1 burial ground [118-N (100-N Area silos)].

6. N Springs roads and boundary.

7. 100-N parking lot.

8. 100-N paved areas inside exclusion area.

9. 100-K URM area [includes: $116-K W-4,116-K E-5,116-K-3$ (outfa11)].

10. 105-K exclusion area (includes: 116-KW-1, 116-KW-2, 116-KE-1, 116-KE-2, $118-K W-2,118-K E-2)$.

11. 100-K flood plain contamination area.

12. 118-K-1 burial ground.

13. 116-K-1 crib.

14. 116-K-2 (mile-long trench).

15. 107-KE (116-KE-4).

16. 107-KW (116-KW-3).

17. 100-B/C URM area [includes 116-B-1,116-B-2,116-B-5, 116-B-7, 132-B-6 $(116-B-8), 116-B-13,116-B-14,116-C-1,116-C-2 A, 132-C-2(116-C-4)$, $118-B-2,118-B-3,118-B-4,118-B-5,118-B-6,118-B-7,116-C-5$, $116-B-11]$.

18. 105-B/C exclusion area (includes 116-B-3, 116-B-4, 116-B-6A, 116-B-6B, $116-B-9,116-B-10,118-C-2,118-C-4,116-C-2 B, 116-C-2 C)$.

19. $100 \mathrm{~B} / \mathrm{C}$ surface contamination area (includes $116-C-5,116-B-11$, 116-B-13).

20. 118-C-1 burial ground.

21. 118-B-1 burial ground. 
WHC-SP-0098-6

This page intentionally left blank. 
WHC-SP-0098-6

OPERATIONS AND MAINTENANCE CONTRACTOR

(WESTINGHOUSE HANFORD COMPANY)

ANNUAL SCHEDULE 
WHC-SP-0098-6

This page intentionally left blank. 
WHC-SP-0098-6

JANUARY

WEEKLY

1. Routine water samples.

BIWEEKLY

1. Routine air samples.

\section{BIMONTHLY}

1. Road surveys inside 200 East Area.

2. Road surveys inside 200 West Area.

\section{QUARTERLY}

1. Tritium and nitrate water samples.

\section{SEMI-ANNUALLY}

1. Composite air samples.

2. Outside perimeter roads of 200 East Area.

3. Outside perimeter roads of 200 West Area.

4. Vent station perimeter and surface contamination area (cross site transfer line).

\section{ANNUALLY}

1. Highway survey (Route 4 South) 1100 Area to Wye Barricade, to include 300 and 400 Area access roads and parking lots.

2. Posted URM pipelines in 200 East Area (excluding cross site transfer line). 
WHC-SP-0098-6

\section{FEBRUARY}

\section{WEEKLY}

1. Routine water samples.

\section{BIWEEKLY}

1. Routine air samples.

\section{QUARTERLY}

1. 244-A lift station perimeter.

\section{SEMI-ANNUALLY}

1. Route 4 south from 200 West main gate to 200 East Area hi11.

\section{ANNUALLY}

1. 216-T-4-2 ditch.

2. 307 retention basin perimeter.

3. $216-C-4$.

4. $216-C-7$.

5. 241-TX-155 diversion box.

6. 241-ER-152 diversion box.

7. Original Central Landfill (Located $2.4 \mathrm{~km}(1.5 \mathrm{mi}$ ) southeast of Army Loop Road, 100 feet north of Highway 4 south].

8. Cross site transfer line (200 East Area section) UN-216-E-41.

9. Route 4 south, 200 East Area hill to Wye Barricade. 
WHC-SP-0098-6

MARCH

\section{WEEKLY}

1. Routine water samples.

\section{BIWEEKLY}

1. Routine air samples.

\section{BIMONTHLY}

1. Survey of roads inside 200 East Area.

2. Survey of roads inside 200 West Area.

\section{QUARTERLY}

1. Thermoluminescent dosimeter (TLD) exchange 100 Area.

2. TLD exchange $200 / 600$ Areas.

3. TLD exchange $300 / 400$ Areas.

4. Waste site visual survey (posting, housekeeping, vegetation control, animal activity, unusual conditions, etc.).

\section{SEMI-ANNUALLY}

1. $200 / 600$ Areas emergency piot surveys.

2. 216-A-30 crib.

3. 216-B-55 crib.

4. 216-B-62 crib.

\section{ANNUALLY}

1. Cross site transfer 1 ine from 200 East fence to access road near vent station (UN-216-E-41).

2. Unplanned release sites in 200 East Area: UN-216-E-7, UN-216-E-8, UN-216-E-10, UN-216-E-13, UN-216-E-15, UN-216-E-18, UN-216-E-24, UN-216-E-26.

3. Highway survey, from entrance to 200 West Area to Route $11 \mathrm{~A}$, Route $11 \mathrm{~A}$ to Route 2 south to Highway 240 via Route 10.

4. 216-Z-1 and -2 crib.

5. 216-Z-1 ditch.

6. 216-Z-3 crib.

7. 216-Z-13 French drain.

8. 216-Z-14 French drain.

9. 216-Z-15 French drain. 
WHC-SP-0098-6

\section{APRIL}

\section{WEEKLY}

1. Routine water samples.

\section{B IWEEKLY}

1. Routine air samples.

\section{QUARTERLY}

1. Tritium and nitrate water samples.

\section{SEMI-ANNUALLY}

1. Railroad survey 300 Area to 400 and 200 West Area, to include all sidings and spurs outside of fence boundaries of areas.

2. 216-U-14 (active section from 207-U to 216-U-10 pond).

\section{ANNUALLY}

1. UN-300-17 (333 bldg).

2. UN-300-XX (333 bldg).

3. $216-A-40$.

4. $216-A-8$.

5. 216-B-13.

6. $216-B-64$.

7. 216-T-1 ditch perimeter.

8. $300 / 400$ Area soil and vegetation sampling.

9. Cross site transfer 7 ine from access road near vent station to 200 West Area fence (UN-216-E-41).

10. Highway survey, Yakima Barricade to Army Loop Road (Route 11A), Army Loop Road to Route 4 south. 


\section{WEEKLY}

1. Routine water samples.

\section{BIWEEKLY}

1. Routine air samples.

\section{BIMONTHLY}

1. Road survey inside 200 East Area.

2. Road survey inside 200 West Area.

\section{QUARTERLY}

1. 244-A 7ift station perimeter.

\section{SEMI-ANNUALLY}

1. 200 West Area tank farm perimeters:
A. 241-T.
B. 241-TX and 241-TY.
C. 241-S, 241-SX, and 241-SY.
D. 241-U.

2. 200 East Area Tank Farm Perimeters:
A. 241-A, 241-AN, 241-AX, 241-AY, and 241-AZ.
B. 241-AP.
C. 241-AW.
D. 241-B.
E. 241-BX and 241-BY.
F. 241-C.

\section{ANNUALLY}

1. 200/600 Areas soil and vegetation sampling.

2. 200/600 Area pond and ditch aquatic vegetation sampling.

3. 200/600 Area pond and ditch sediment sampling.

4. Highway survey, $100-\mathrm{N}$ Area to intersection of Route $11 \mathrm{~A}$ (Route $2 \mathrm{~N}$ ) to include all parking areas, paved access roads to 100 Areas, and Route 1 from Hanford townsite to 100-B Area.

5. Rail survey 200 West perimeter fence to $100-\mathrm{K}$ and $100-\mathrm{N}$, including the lower route to May Junction. This survey is to include all sidings and spurs.

6. UN-216-E-9 (UN-200-E-81). 
WHC-SP-0098-6

\section{JUNE}

\section{WEEKLY}

1. Routine water samples.

\section{BIWEEKLY}

1. Routine air samples.

\section{QUARTERLY}

1. TLD exchange 100 Area.

2. TLD exchange $200 / 600$ Areas.

3. TLD exchange $300 / 400$ Areas.

4. Waste site visual survey (posting, housekeeping, vegetation control, animal activity, unusual conditions, etc.).

\section{ANNUALLY}

1. Highway survey, West Lake to Route 6 (100-B cut-off), Route 6 to Route $11 \mathrm{~A}$, Route $11 \mathrm{~A}$ to Intersection of Route 3.

2. Rail survey, 200 West Area railways, spurs, and sidings.

3. 216-U-16 crib.

4. $216-\mathrm{S}-25 \mathrm{crib}$.

5. $216-\mathrm{S}-26 \mathrm{crib}$.

6. 216-U-17 crib.

7. 400 Area process pond perimeters.

8. 116-K-3.

9. 116-KE-6A.

10. 116-KE-6B.

11. 116-KE-6C.

12. 116-KE-6D.

13. 218-E-13 PUREX tunnel perimeter.

14. 218-E-14 PUREX tunnel perimeter.

15. UN-216-E-25 (UPR-200-E-75). 
WHC-SP-0098-6

JULY

\section{WEEKLY}

1. Routine water samples.

\section{BIWEEKLY}

1. Routine air samples.

\section{BIMONTHLY}

1. Road surveys inside 200 East Area.

2. Road surveys inside 200 West Area.

\section{QUARTERLY}

1. Tritium and nitrate water samples.

\section{SEMI-ANNUALLY}

1. Composite air samples.

2. Outside perimeter roads of 200 East Area.

3. Outside perimeter roads of 200 West Area.

4. Vent station perimeter and surface contamination area (cross site transfer line).

\section{ANNUALLY}

1. 216-Z-9.

2. 207-B retention basin perimeter.

3. 207-SL retention basin perimeter.

4. 207-T retention basin perimeter (includes $216-T-12$ sludge pit).

5. 207-U retention basin perimeter (includes $U N-216-W-21$ and $U N-216-W-22$ ).

6. 216-A-42 retention basin perimeter (207-aa).

7. 100 Area soil and vegetation sampling.

8. Highway survey, from intersection of Route 3 and Route 4 north to $100-\mathrm{N}$.

9. Rail survey, 200 East Area railways, spurs, and sidings.

10. Manhole covers along supply and discharge Iines to the 300 Area Treated Effluent Disposal Facility.

11. UN-216-E-22 (UPR-200-E-94). 
WHC-SP-0098-6

\section{AUGUST}

\section{WEEKLY}

1. Rout ine water samples.

\section{BIWEEKLY}

1. Routine air samples.

\section{QUARTERLY}

1. 244-A 1 ift station perimeter.

\section{SEMI-ANNUALLY}

1. Route 4 south from 200 West Area main gate to 200 East Area hill.

\section{ANNUALLY}

1. UN-300-5 (309 tank farms).

2. UN-200-W-8 (old burial ground east of U Plant).

3. 216-B-63 ditch survey.

4. Unplanned release site surveys 200 West Area: UN-216-W-4, UN-216-W-6, $\mathrm{UN}-216-\mathrm{W}-12$, and $\mathrm{UN}-216-\mathrm{W}-28$.

5. Rail survey, 300 Area to Columbia Center to include all sidings and spurs.

6. 216-B-3C pond perimeter (active). 
WHC-SP-0098-6

\section{SEPTEMBER}

\section{WEEKLY}

1. Routine water samples.

\section{BIWEEKLY}

1. Routine air samples.

\section{BIMONTHLY}

1. Survey of roads inside 200 East Area.

2. Survey of roads inside 200 West Area.

\section{QUARTERLY}

1. TLD exchange - 100 Area.

2. TLD exchange - $200 / 600$ Areas.

3. TLD exchange - $300 / 400$ Areas

4. Waste site visual survey (posting, housekeeping, vegetation control, animal activity, unusual conditions, etc.).

\section{SEMI-ANNUALLY}

1. $200 / 600$ Areas emergency plot surveys.

2. $216-\mathrm{A}-30 \mathrm{crib}$.

3. 215-B-55 crib.

4. 216-B-62 crib.

\section{ANNUALLY}

1. N Springs water sampling.

2. Unplanned release site survey 200 West Area: UN-216-E-5, UN-216-E-6, UN-216-E-12 perimeter, UN-216-E-14, UN-216-E-16, UN-216-E-23, UN-216-E-30, UN-216-E-34, UN-216-E-36, UN-216-E-43, and UN-216-E-44.

3. 216-A-45 crib. 
WHC-SP-0098-6

\section{OCTOBER}

\section{WEEKLY}

1. Routine water samples.

\section{BIWEEKLY}

1. Routine air samples.

\section{QUARTERLY}

1. Tritium and nitrate water samples.

\section{SEMI-ANNUALLY}

1. Railroad survey, 300 Area to 400 and 200 West Areas, to include a 11 sidings and spurs external of fence boundaries of areas.

2. 216-U-14 (active section from 207-U to 216-U-10 pond).

\section{ANNUALLY}

1. UN-300-11 (east of 340 building), UN-216-W-18, UN-216-W-33, UN-216-W-35, UN-216-W-36, UN-216-W-37 and 241-WR vault (includes UN-216-W-9 and UN-216-W-11), 200 West section of cross site transfer line (UN-216-E-).

2. Posted URM pipelines in 200 West Area (excluding east/west transfer line). 
WHC-SP-0098-6

\section{NOVEMBER}

\section{WEEKLY}

1. Routine water samples.

\section{BIWEEKLY}

1. Routine air samples.

\section{BIMONTHLY}

1. Road surveys inside 200 East Area.

2. Road surveys inside 200 West Area.

\section{QUARTERLY}

1. 244-A 1ift station perimeter.

\section{SEMI-ANNUALLY}

1. 200 West Area tank farm perimeters:
A. 241-T.
B. 241-TX and 241-TY.
C. 241-S, 241-SX, and 241-SY.
D. 241-U.

2. 200 East Area tank farm perimeters:
A. 241-A, 241-AN, 241-AX, 241-AY, and 241-AZ.
B. 241-AP.
C. 241-AW.
D. 241-B.
E. 241-BX and 241-BY.
F. 241-C.

\section{ANNUALLY}

1. Outside perimeters of a 71 active West Area burial grounds (218-W-3A, 218-W-3AE, 218-W-4B, 218-W-4C, 218-W-5).

2. Outside perimeters of all active East Area burial grounds (218-E-12B, 218-E-15). 
WHC-SP-0098-6

\section{DECEMBER}

\section{WEEKLY}

1. Routine water samples.

\section{BIWEEKLY}

1. Routine air samples.

\section{QUARTERLY}

1. TLD exchange - 100 Area.

2. TLD exchange - $200 / 600$ Areas.

3. TLD exchange - 300/400 Areas.

4. Waste site visual survey (posting, housekeeping, vegetation control, animal activity, unusual conditions, etc.).

\section{ANNUALLY}

1. UN-300-4 (321 bldg).

2. UN-300-40 (303-F pipe trench).

3. 216-B-3C pond perimeter.

4. Special surveys (e.g., fresh animal feces, pipelines, etc.), contact Near-Field Monitoring. 
WHC-SP-0098-6

APPENDIX A

SURVEY SITES AND SAMPLE LOCATIONS

A-1 
WHC-SP-0098-6

This page intentionally left blank. 
Figure A-1. Hanford Site.

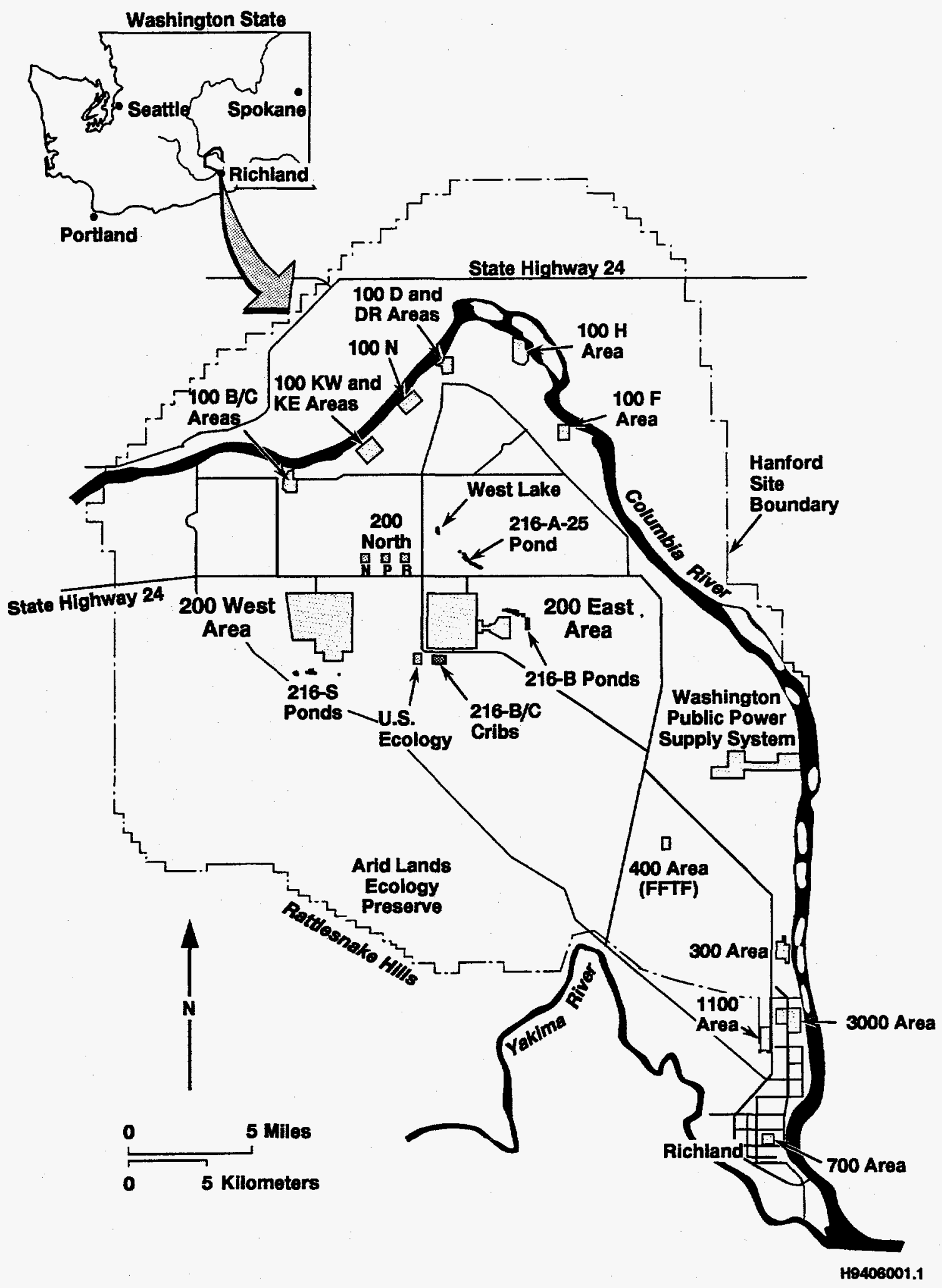


WHC-SP-0098-6

Figure $A-2, \quad 100-B$ and 100-C Waste Sites.

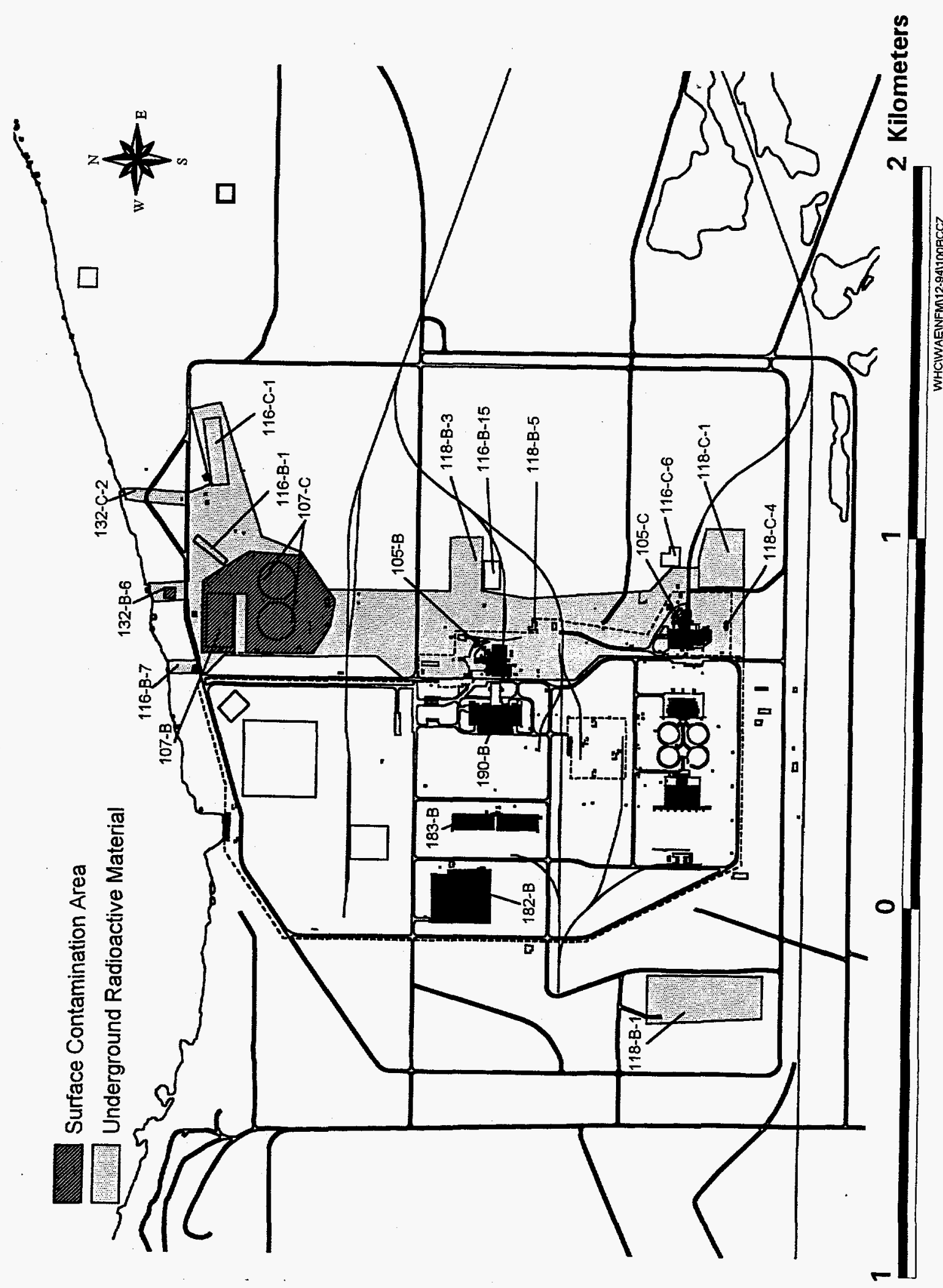




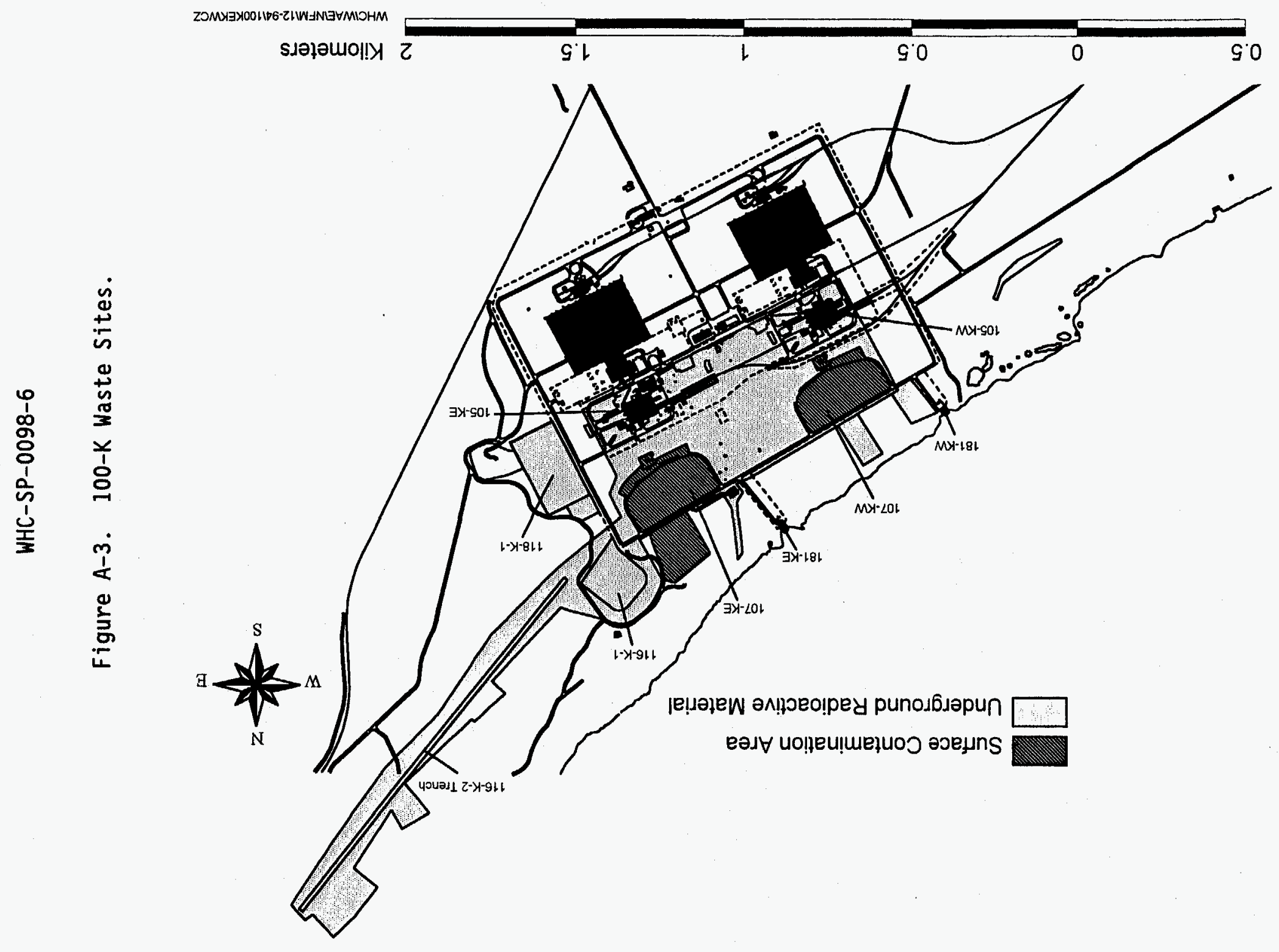




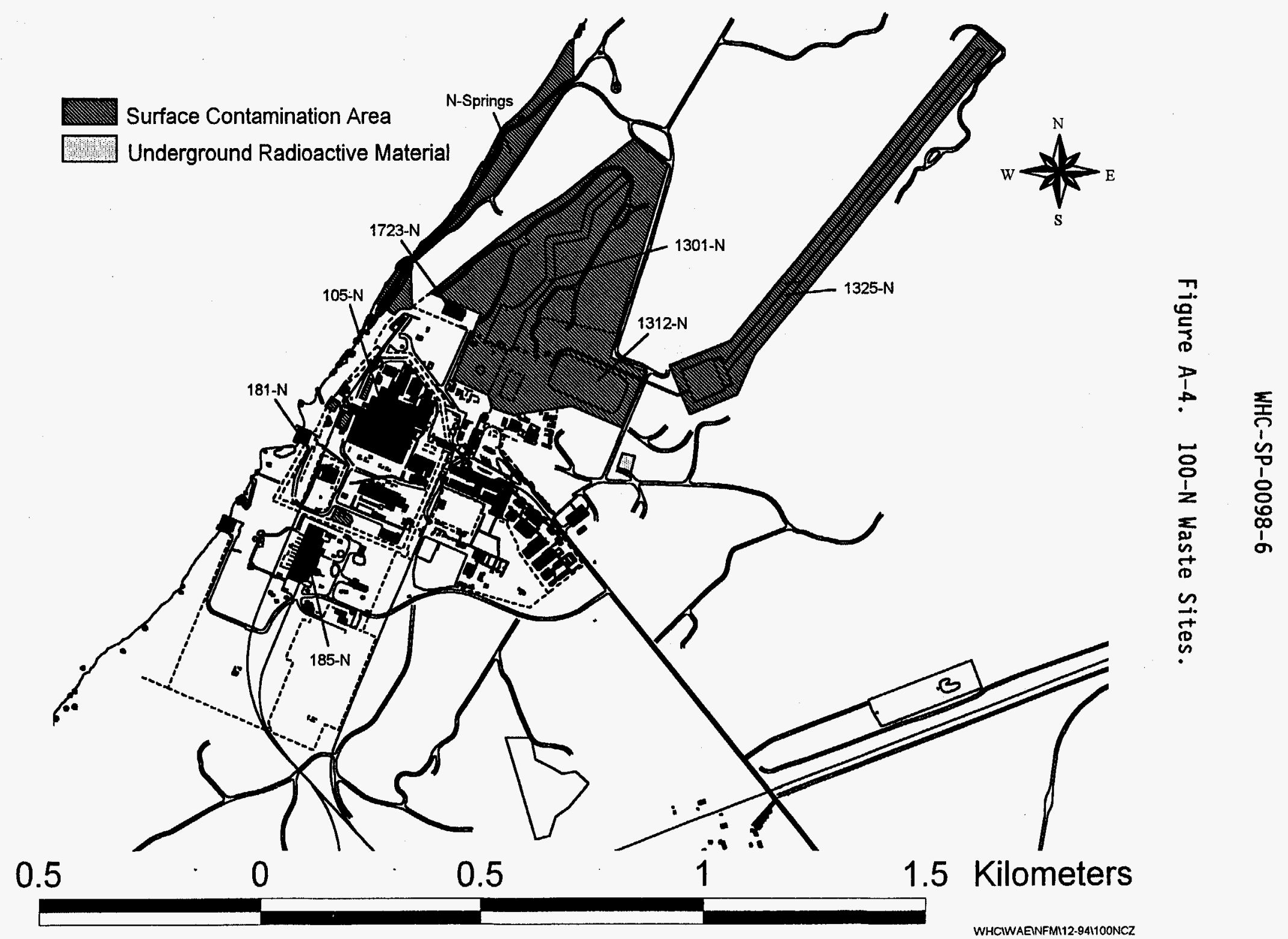


WHC-SP-0098-6

Figure A-5. 100-D Waste Sites.

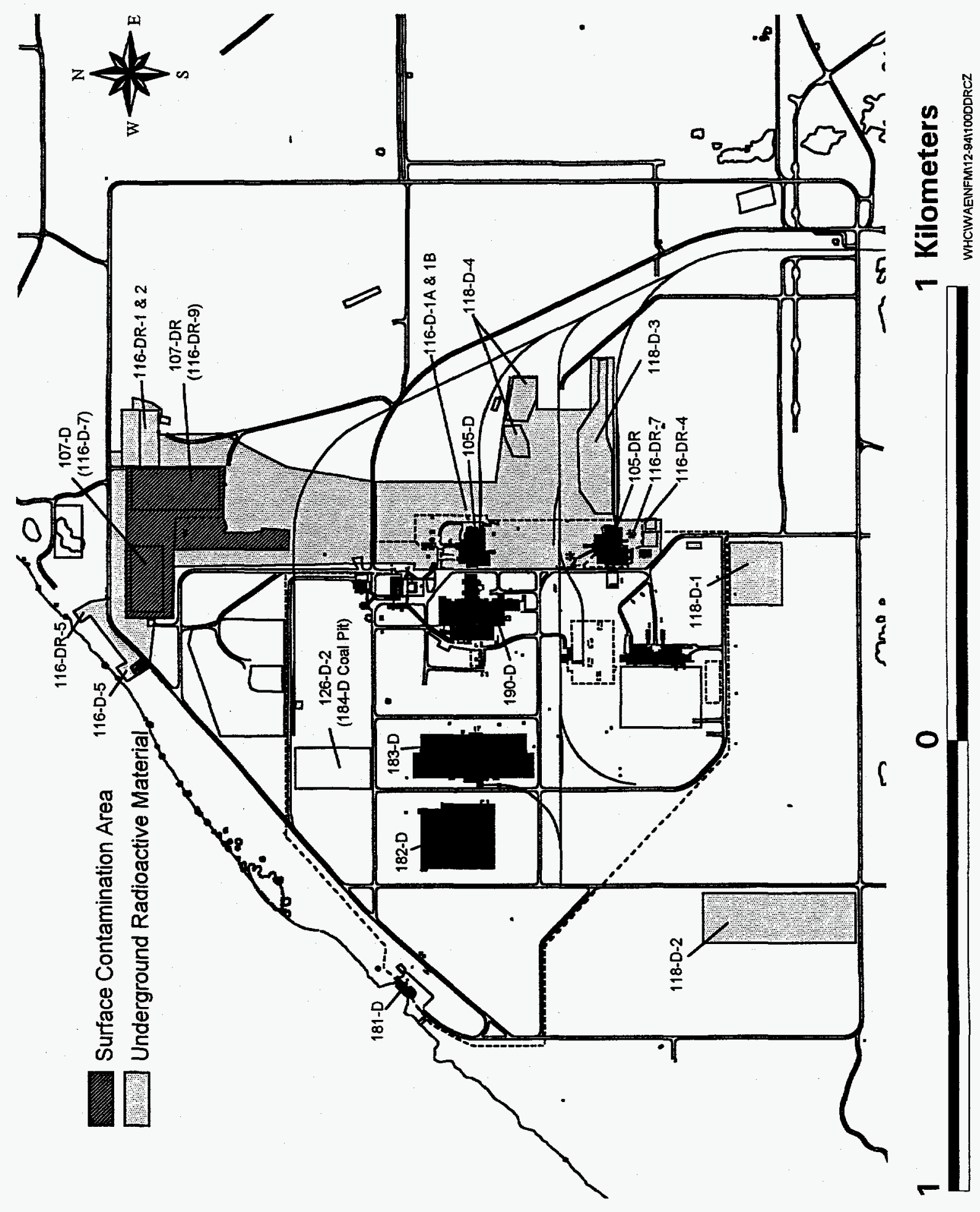


WHC-SP-0098-6

Figure A-6. 100-H Waste Sites.

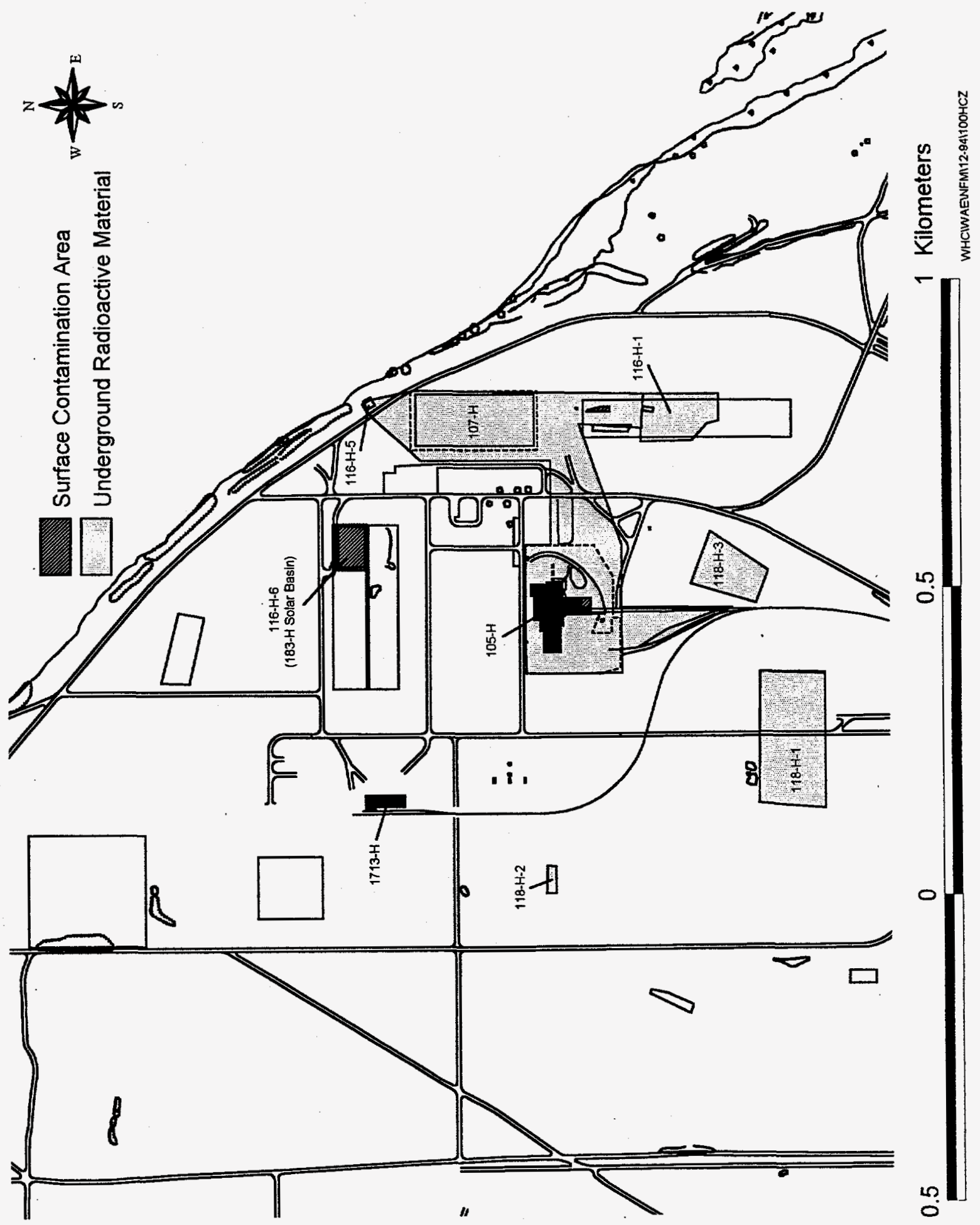



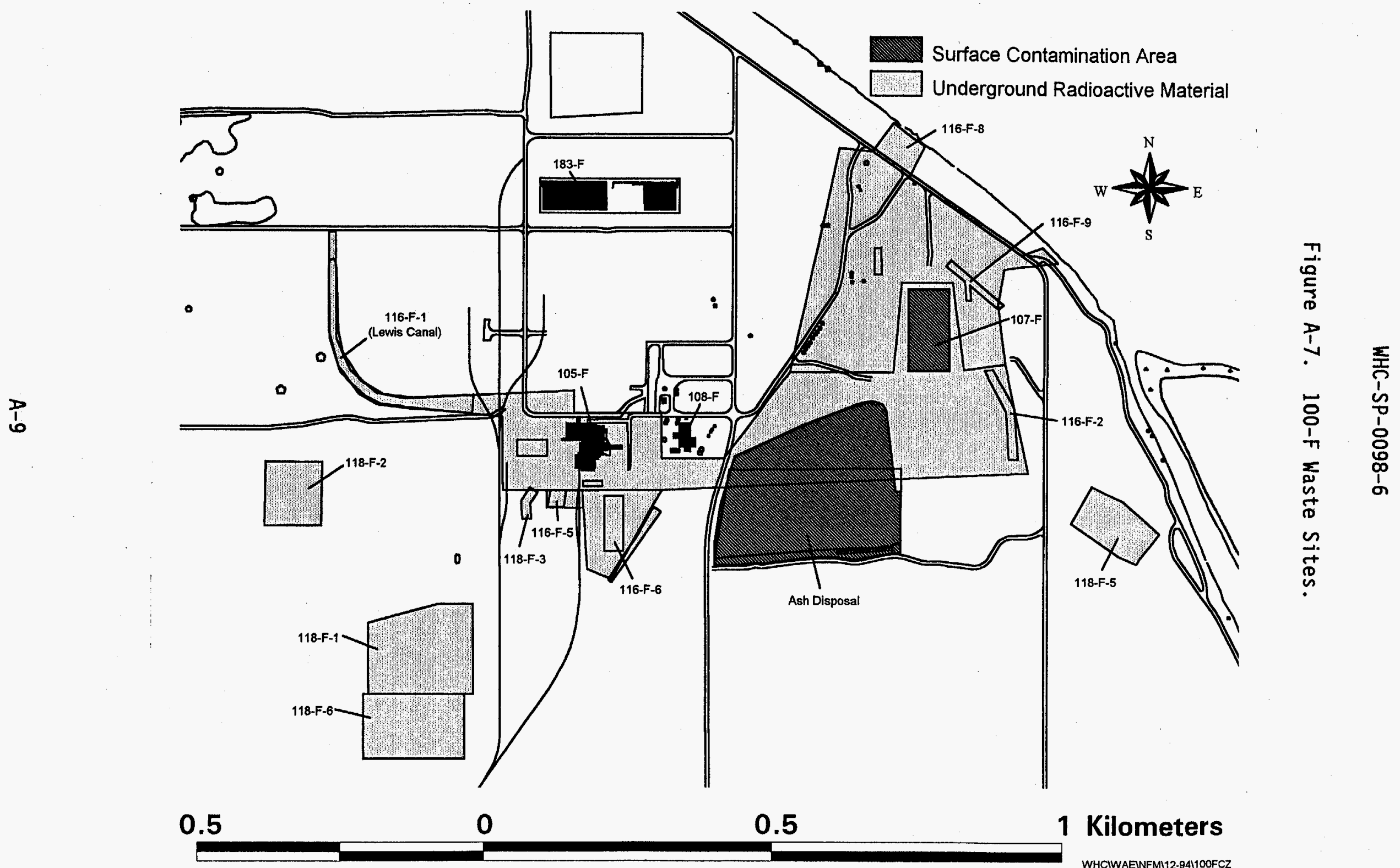
WHC-SP-0098-6

This page intentionally left blank. 
WHC-SP-0098-6

Figures A-8 through A-17 show 200 Areas waste sites. 
WHC-SP-0098-6

Figure A-8. 200 East Waste Sites.
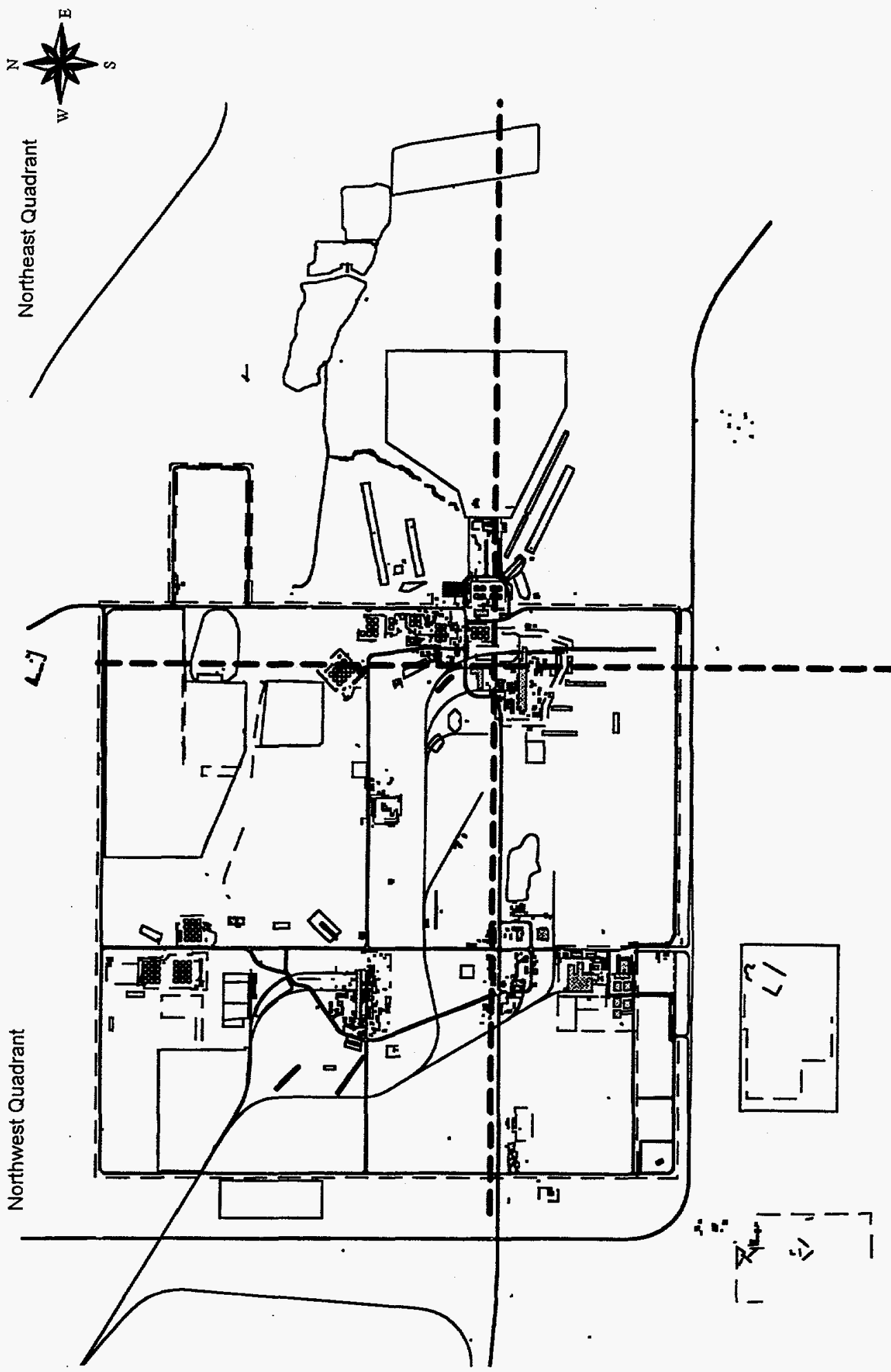

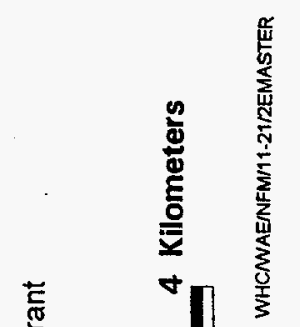

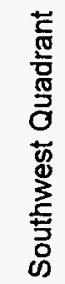

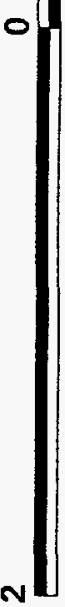


Figure A-9. 200 East Waste Sites, NE Quadrant.
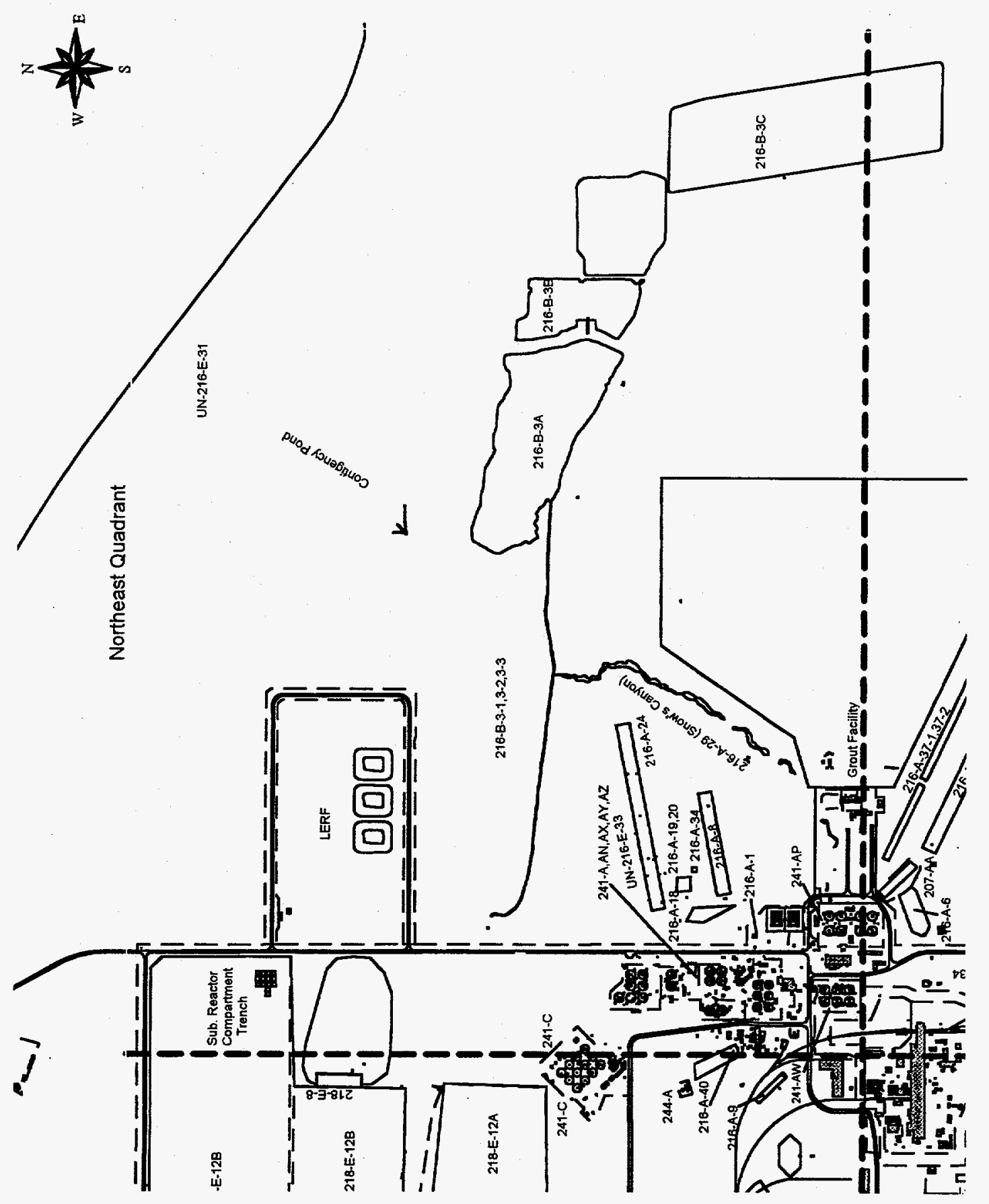
Figure A-10. 200 East Waste Sites, NW Quadrant.

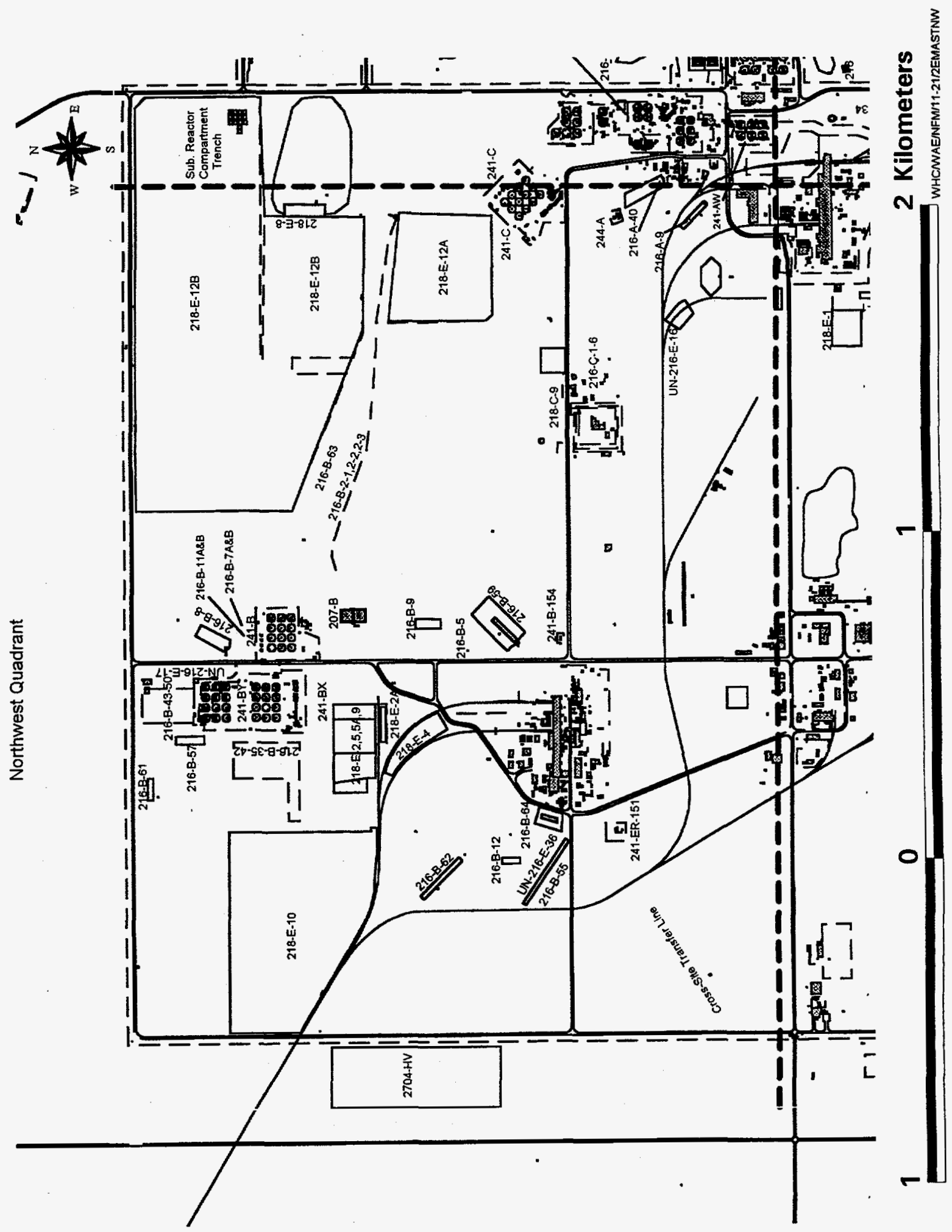


WHC-SP-0098-6

Figure A-11. 200 East Waste Sites, SE Quadrant.

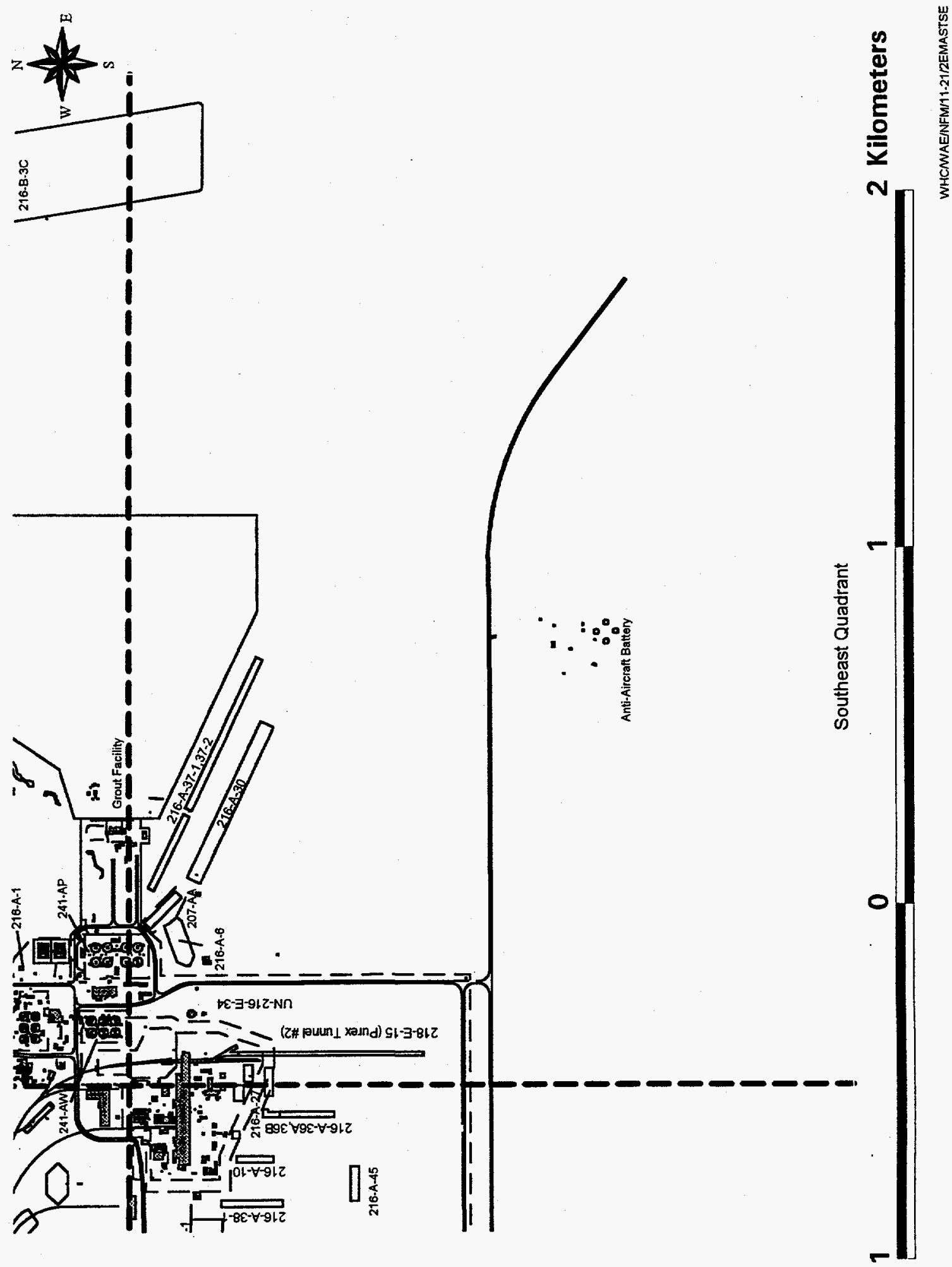


WHC-SP-0098-6

Figure A-12. 200 East Waste Sites, SW Quadrant.

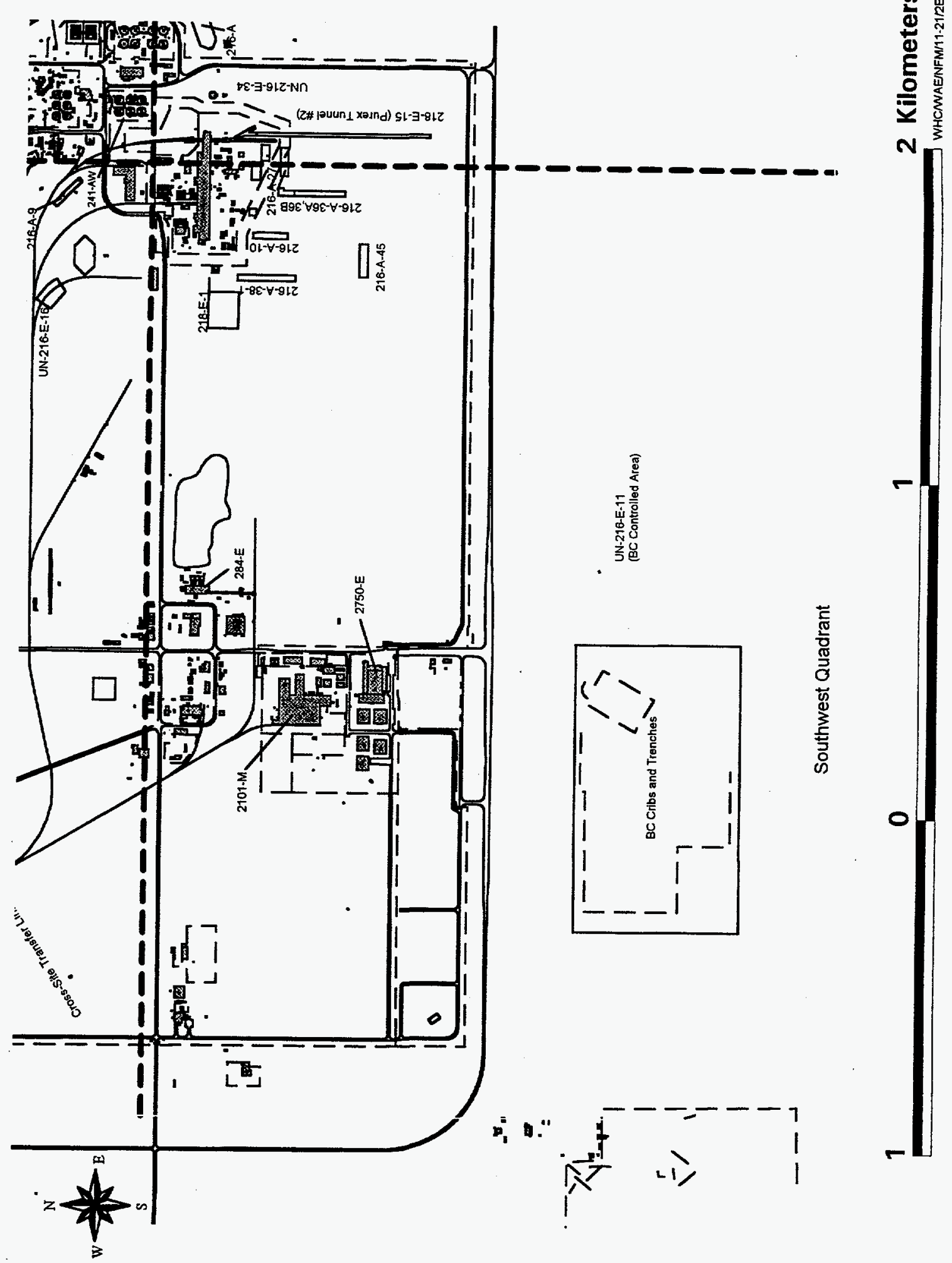


WHC-SP-0098-6

Figure A-13. 200 West Waste Sites.

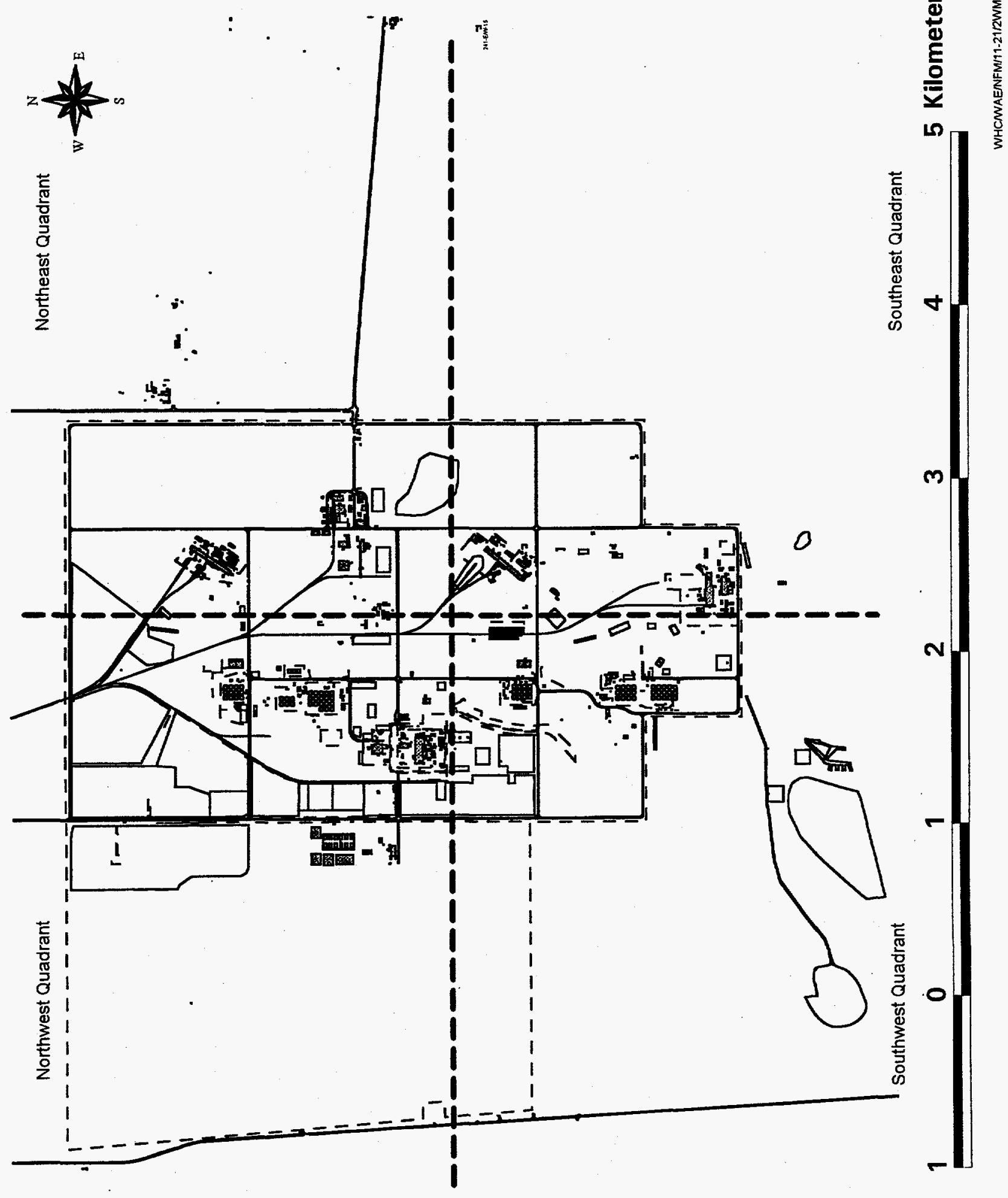


Figure A-14. 200 West Waste Sites, NE Quadrant.

*

$\frac{0}{5}$
$\frac{10}{0}$
$\frac{\pi}{3}$
0
0
$\frac{0}{0}$
0
$\frac{5}{5}$
$\frac{5}{2}$
2

$\int$
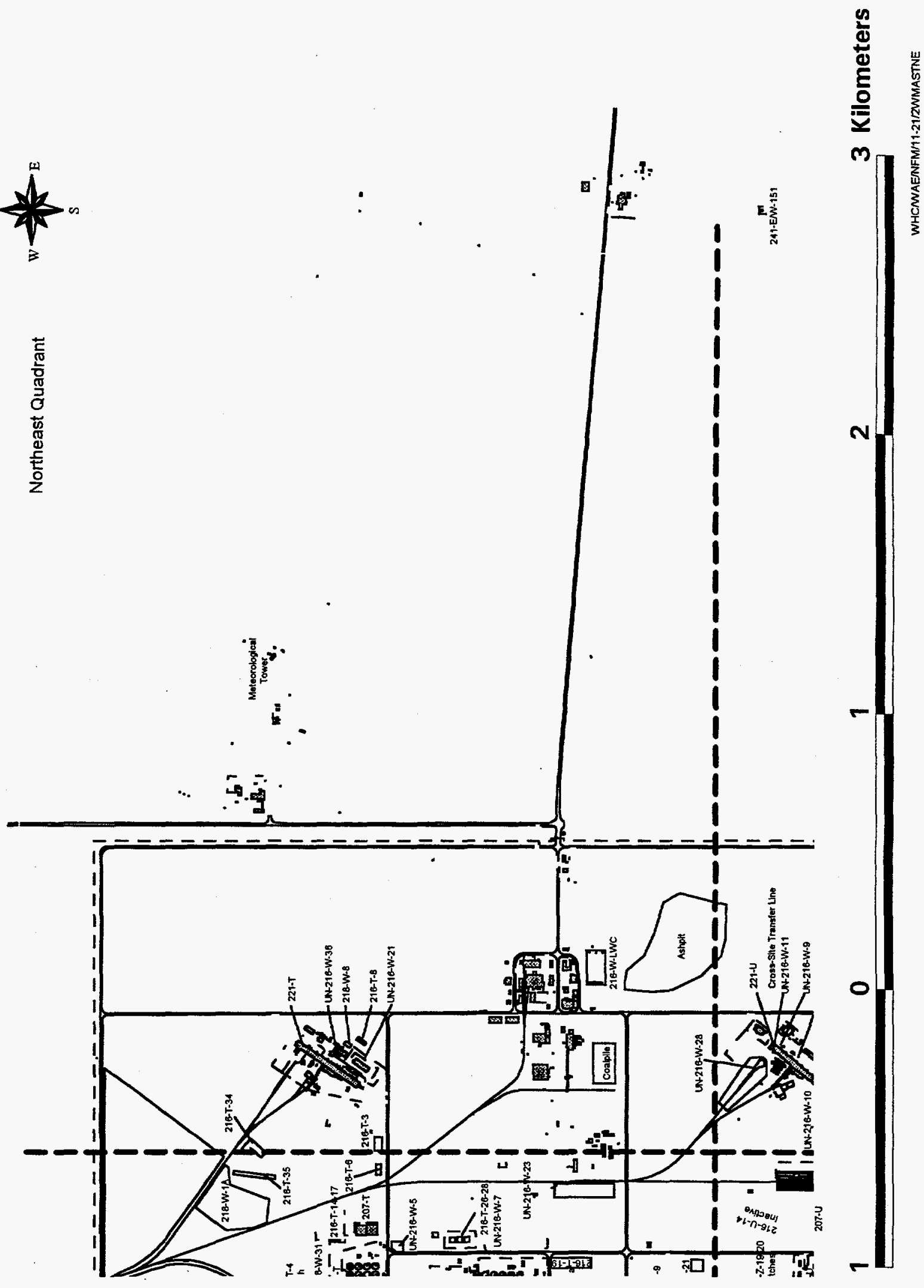
Figure A-15. 200 West Waste Sites, NW Quadrant.

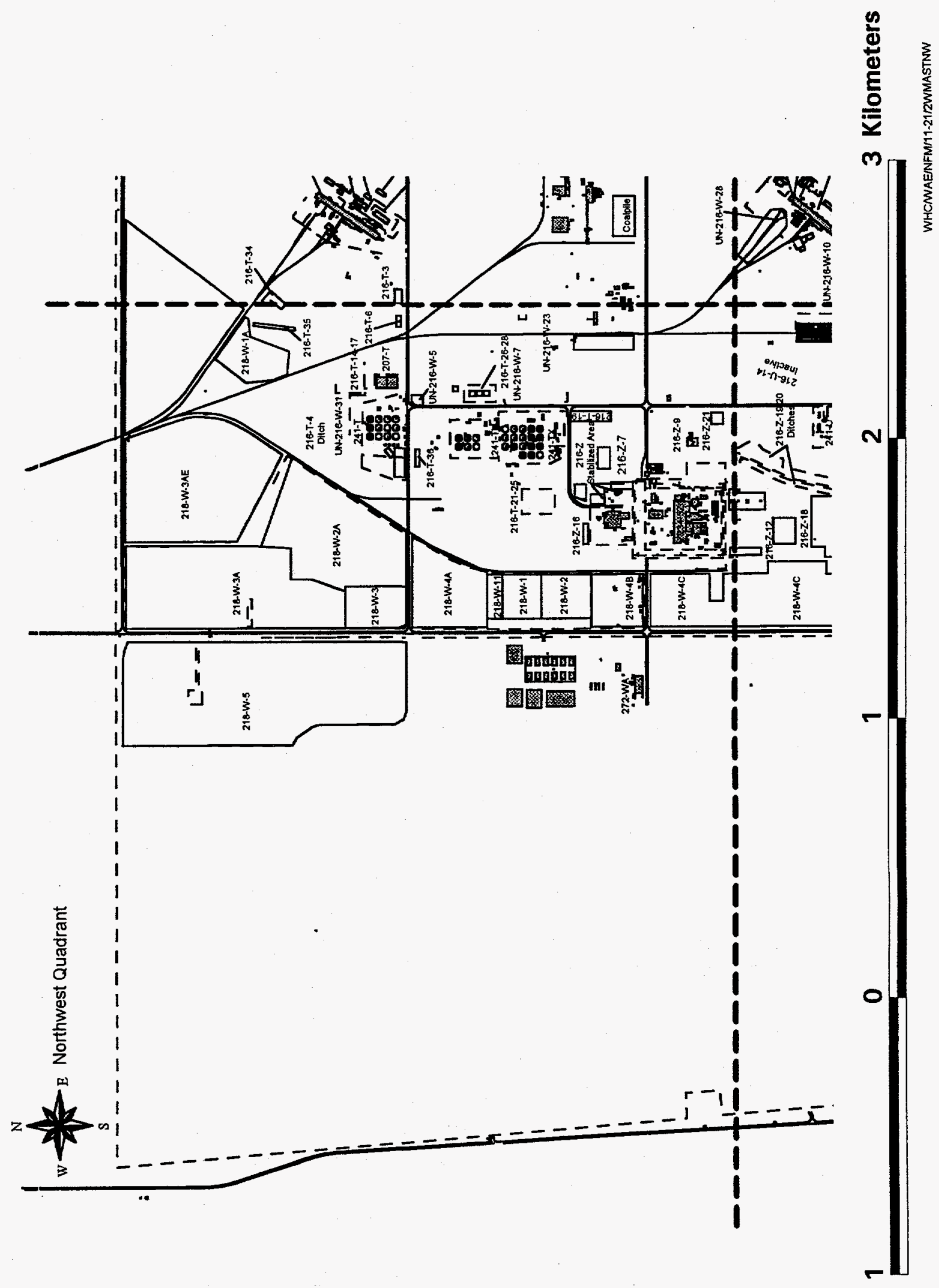


WHC-SP-0098-6

Figure A-16. 200 West Waste Sites, SE Quadrant.

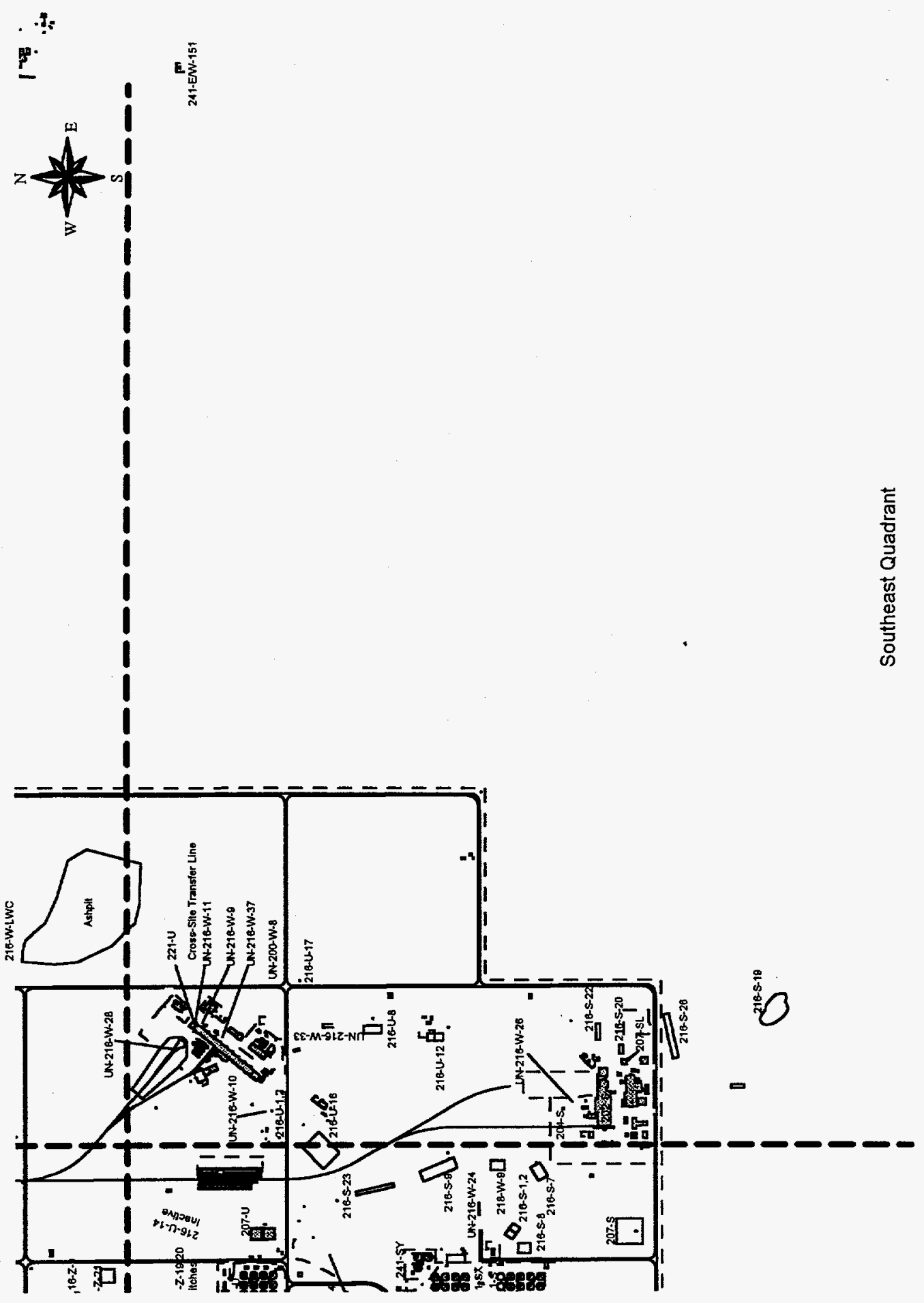


WHC-SP-0098-6

Figure A-17. 200 West Waste Sites, SW Quadrant.

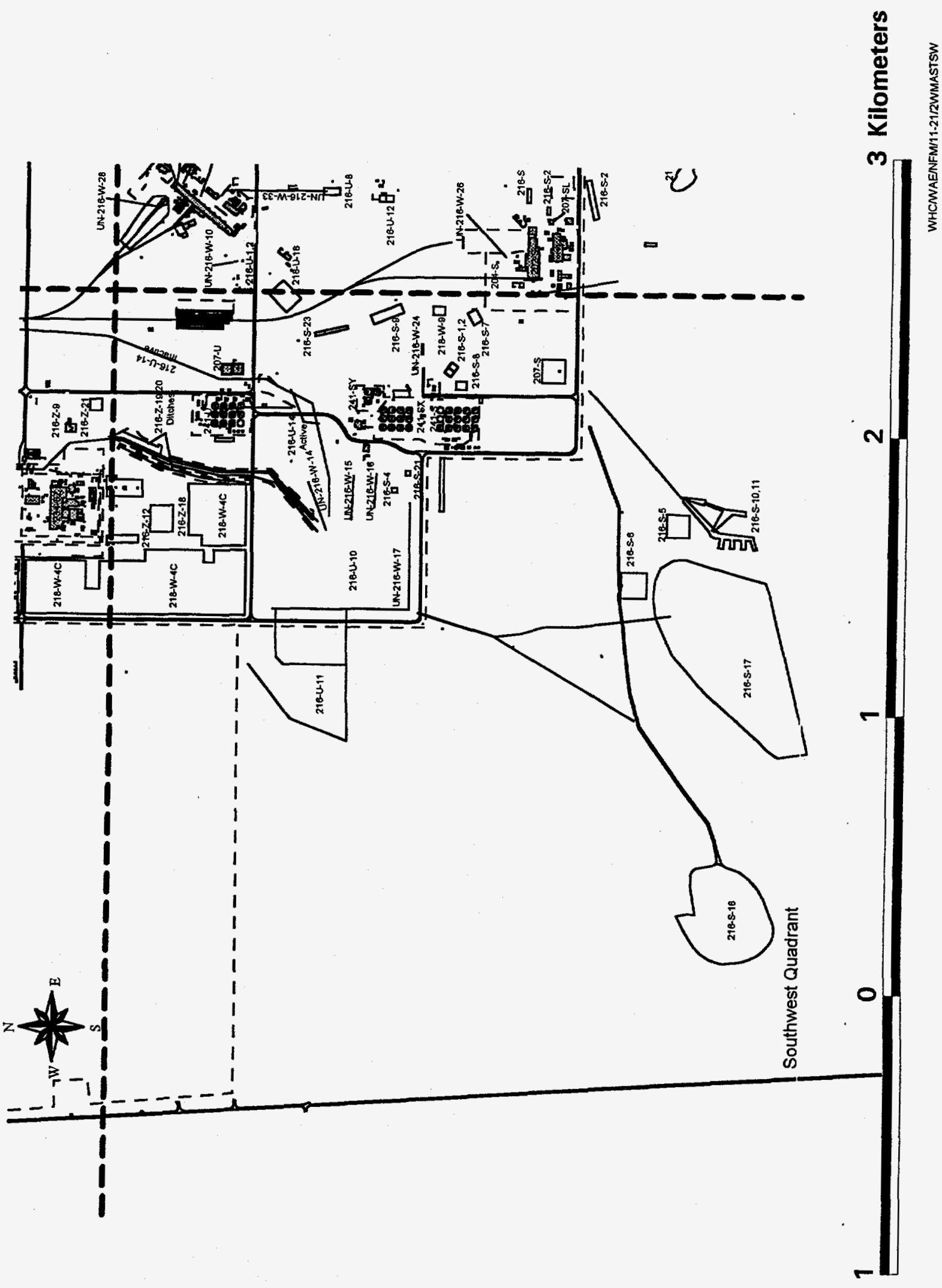


WHC-SP-0098-6

This page intentionally left blank. 


\section{Waste Management Facilities 200 East}

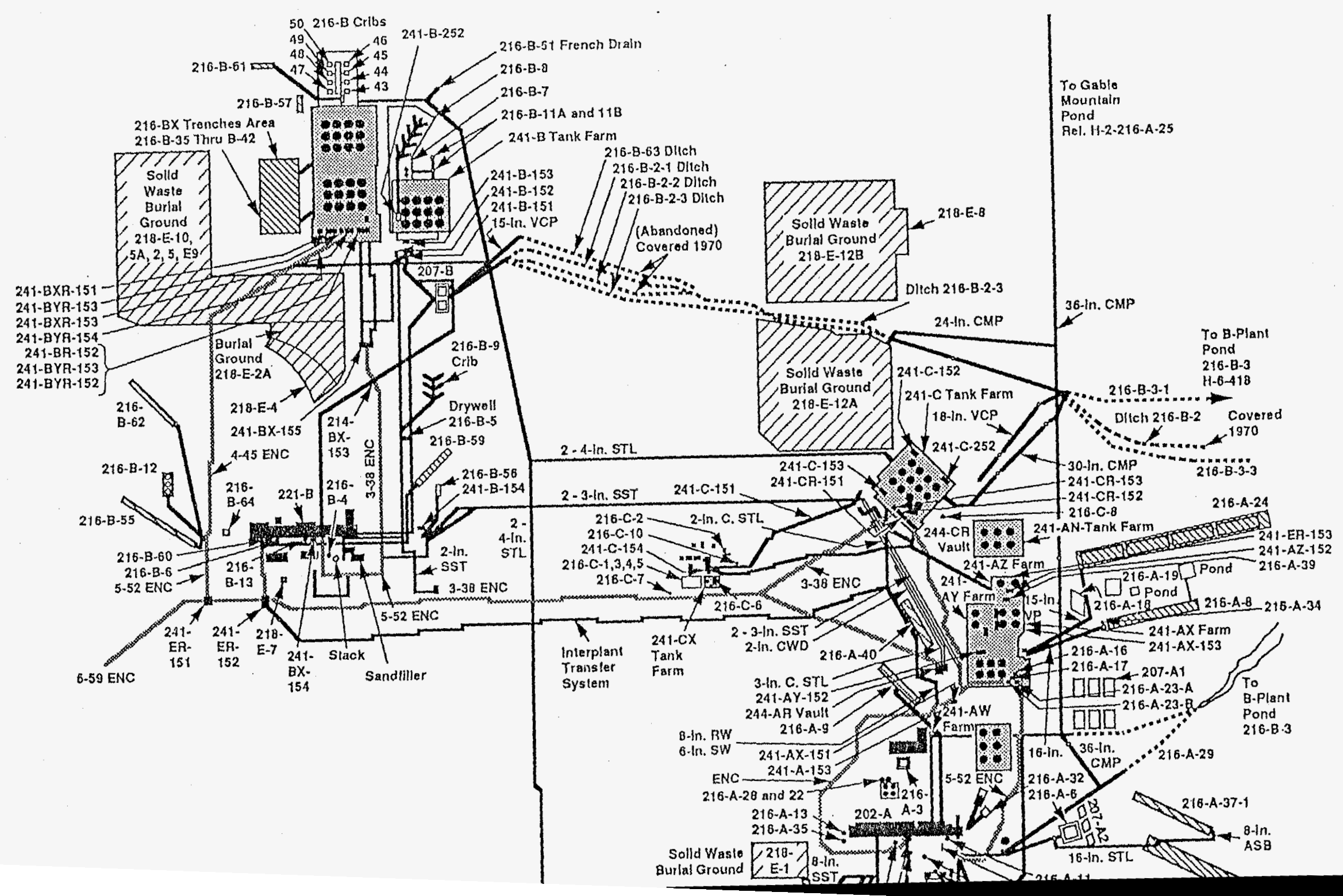


216-A-5 $\frac{B-10^{4}}{216-1} / \int_{216-A-2}^{216-A-A}$

$216-A-12)$ 216-A-27

$216-A-36-B$

216-E-15

77218 - Solid Waste Burial Site

216 - Liquid Waste Burial Site

Arench

- Dry Well or French Drain

216-B-31

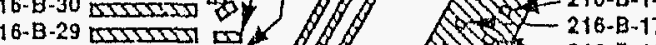

216-8-29

216-B-33 216-B-19

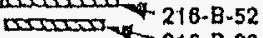

216- $B-18$

92 241-Tank Farm

$\times 216 \cdot 8-23$

$216 \cdot-3-24$

$216-\mathrm{B}-25$

$216 \cdot-8-26$

$216-8 \cdot 28$

Notes:

1) All Facilities are Radioactive

Unless Marked Otherwise.

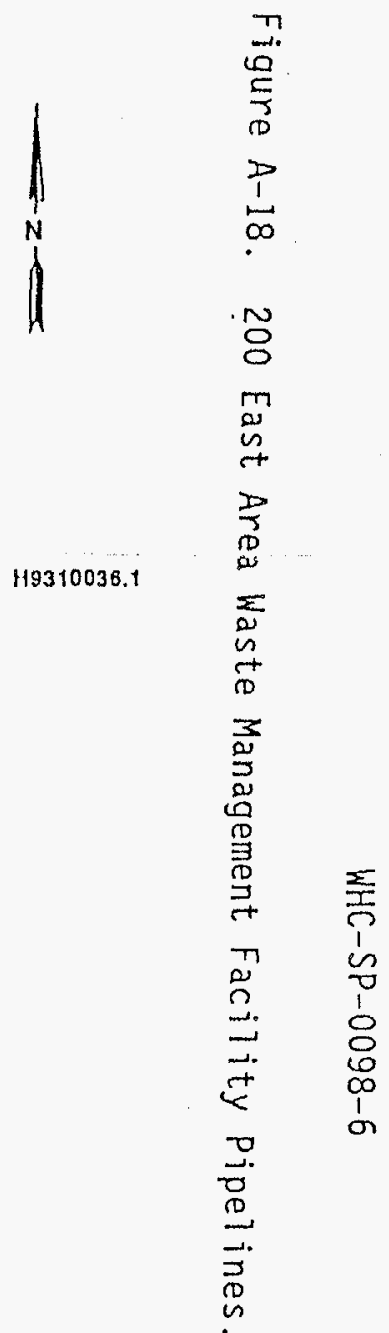




\section{Waste Management Facilities 200 West}

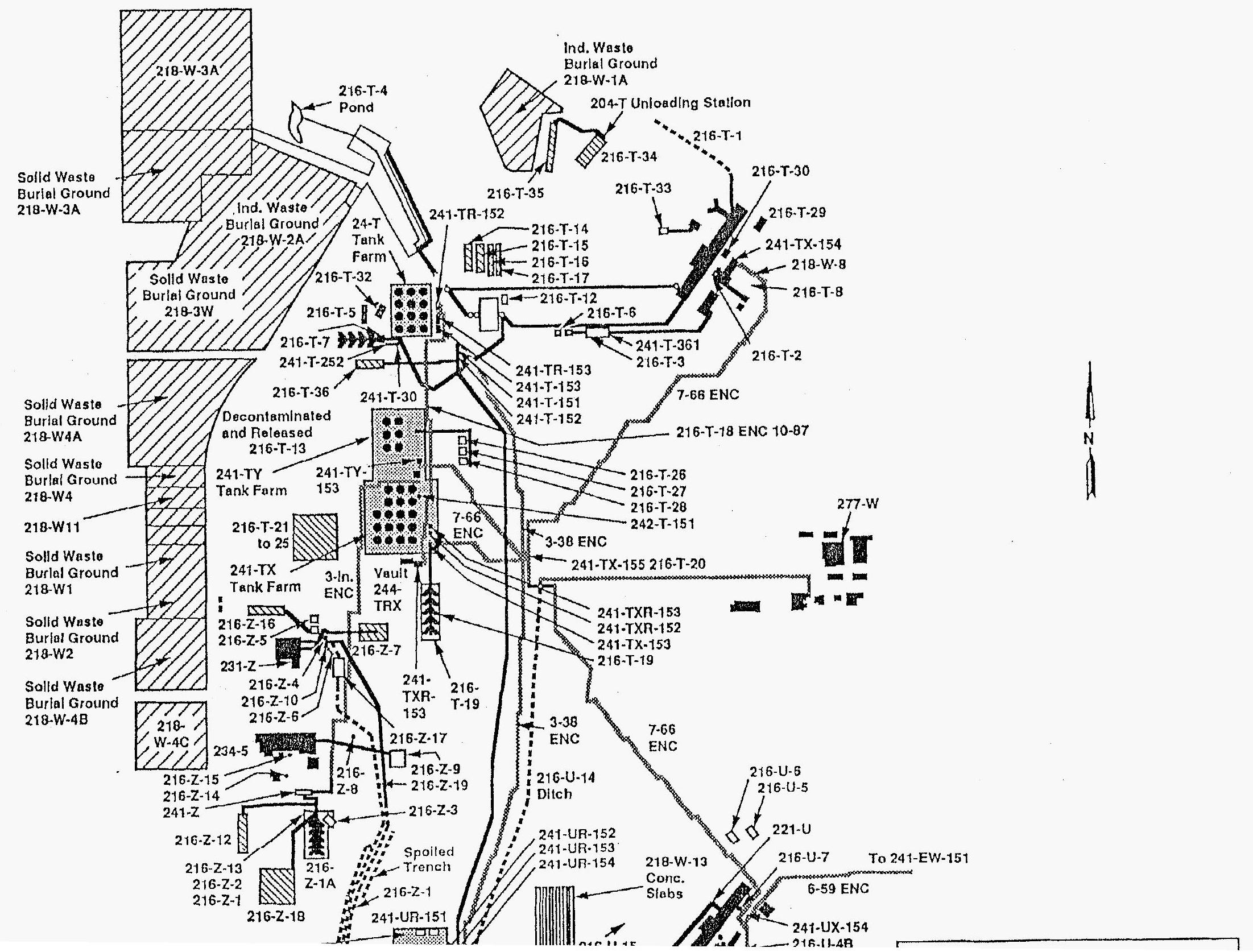




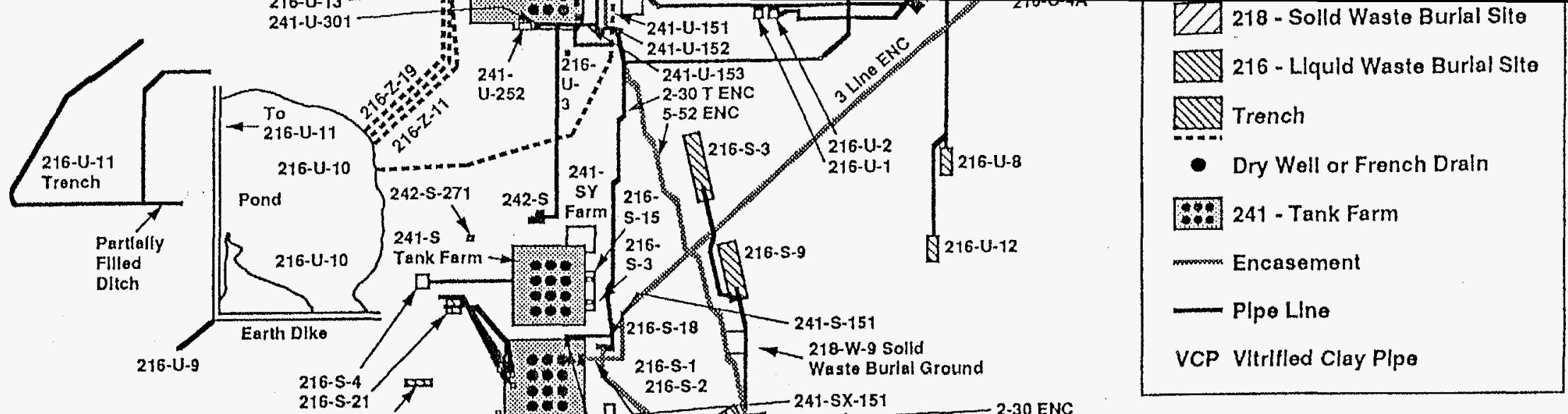

$\frac{P}{1}$
$\tilde{N}$
$\frac{1}{1}$
$\tilde{N}$
or 
WHC-SP-0098-6

Figure A-20. 300 Area Waste Sites.

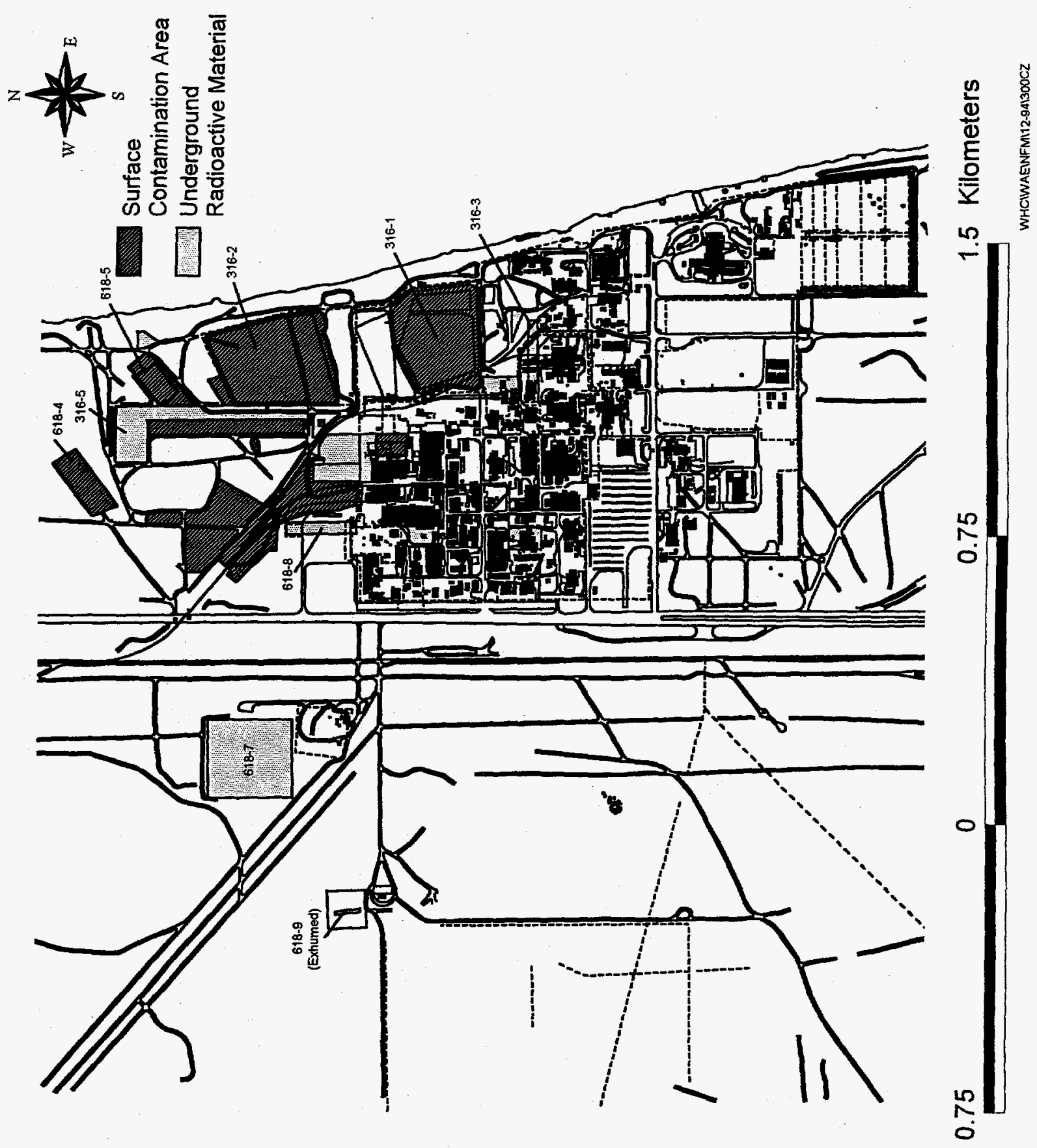


WHC-SP-0098-6

Figure A-21. 400 Area Waste Sites.

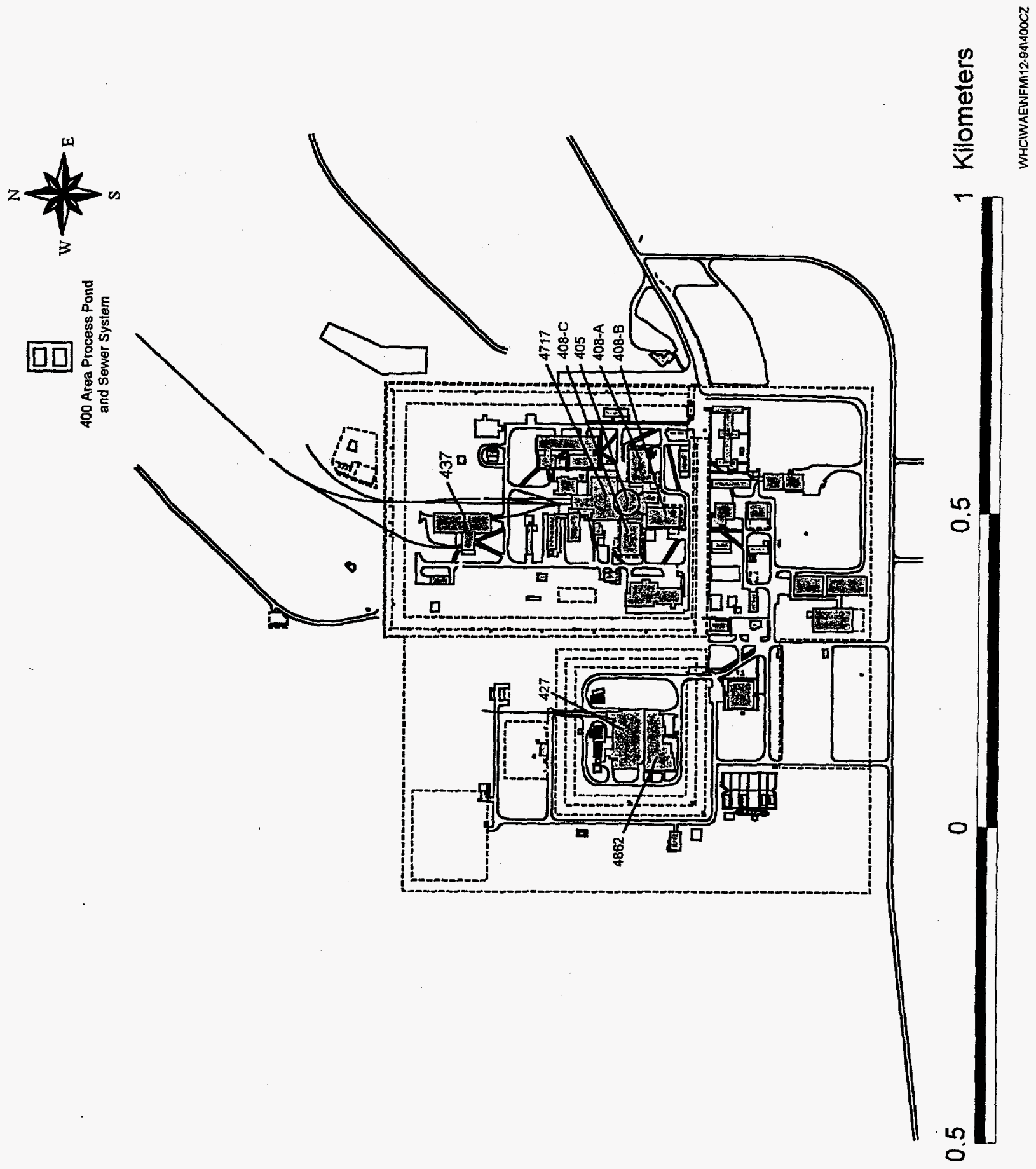


WHC-SP-0098-6

Figure A-22. 600 Area Waste Sites.

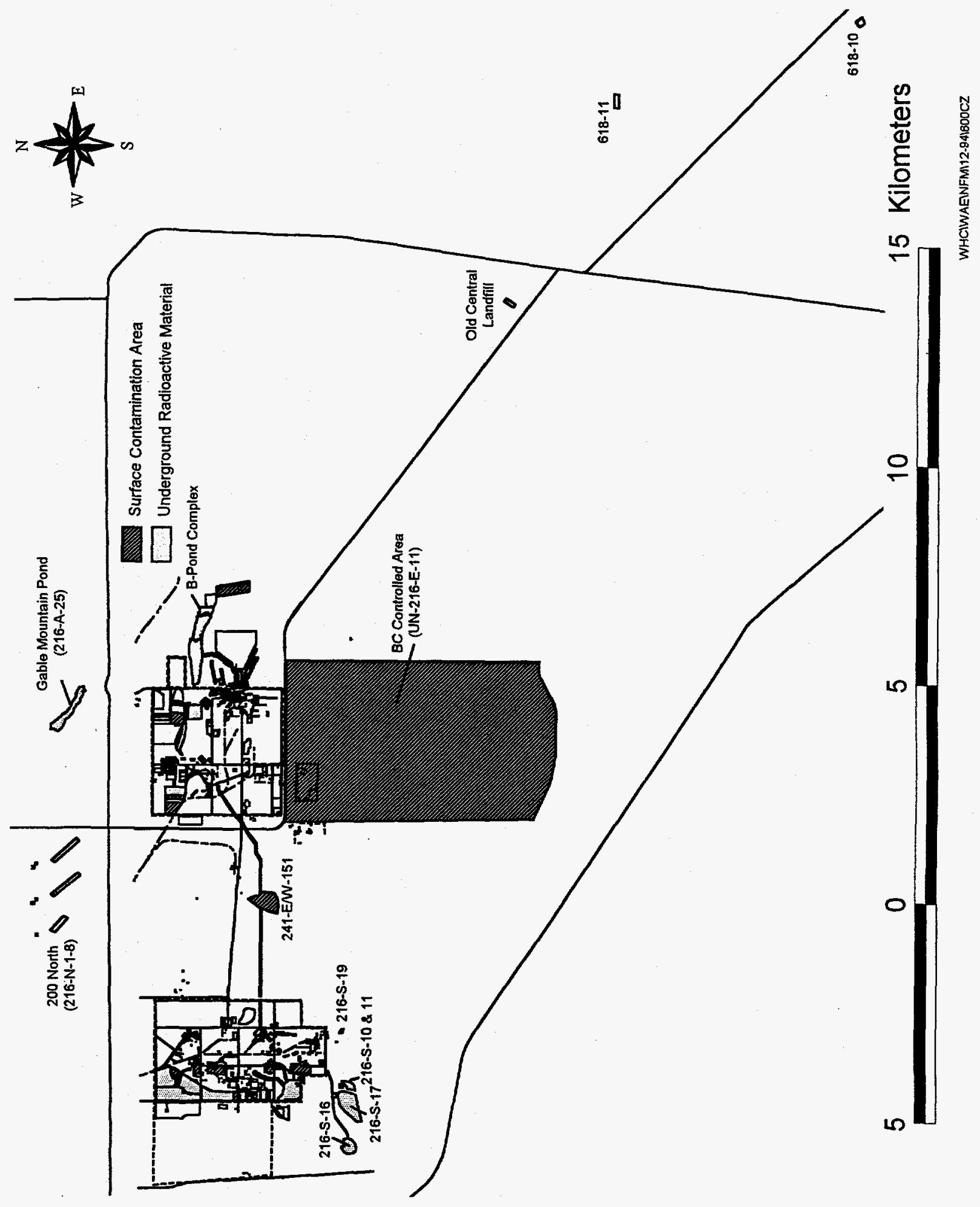


WHC-SP-0098-6

Figure A-23. Site Highways and Railways.

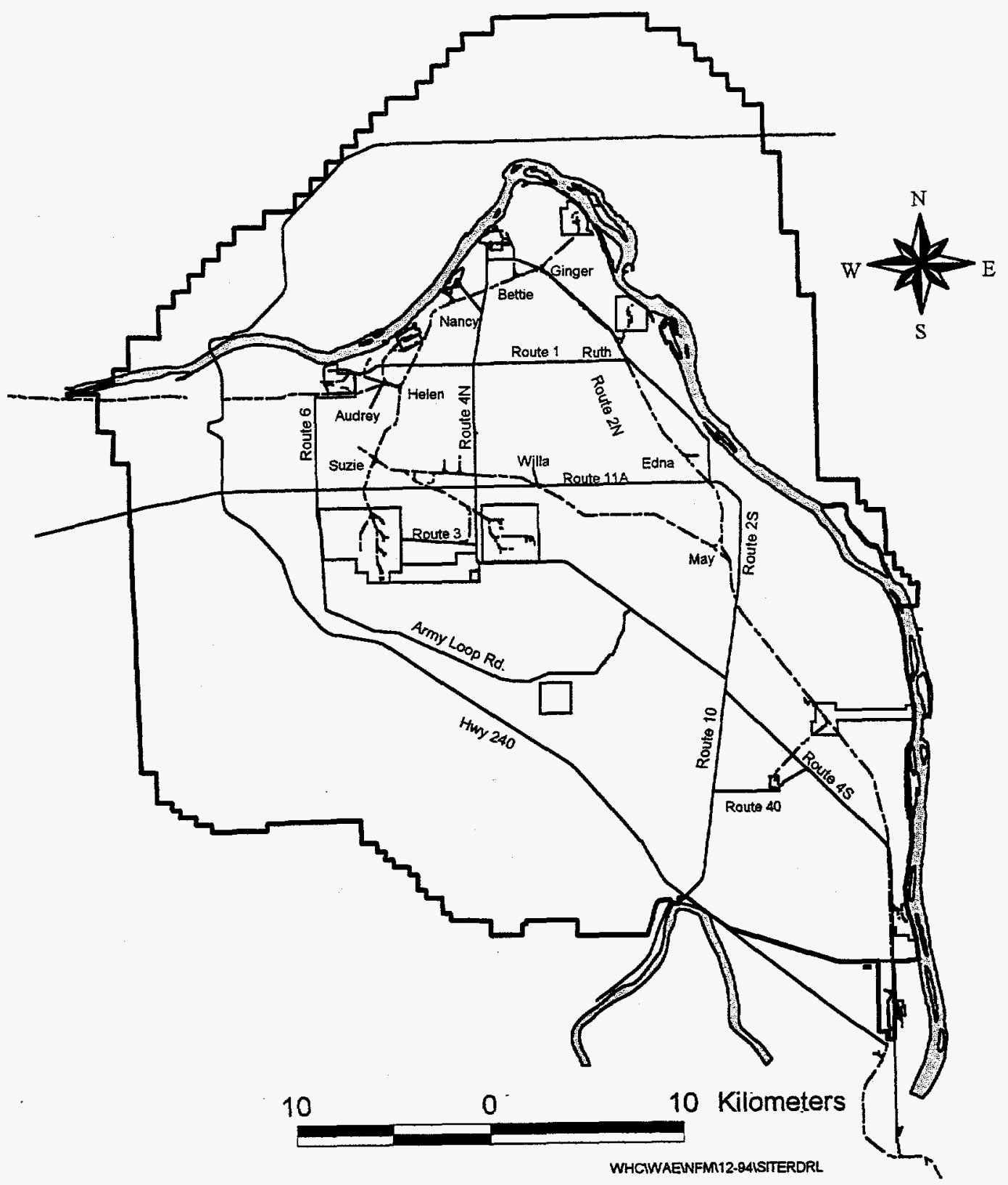


Figure A-24. 200 East Area Roads and Railways.

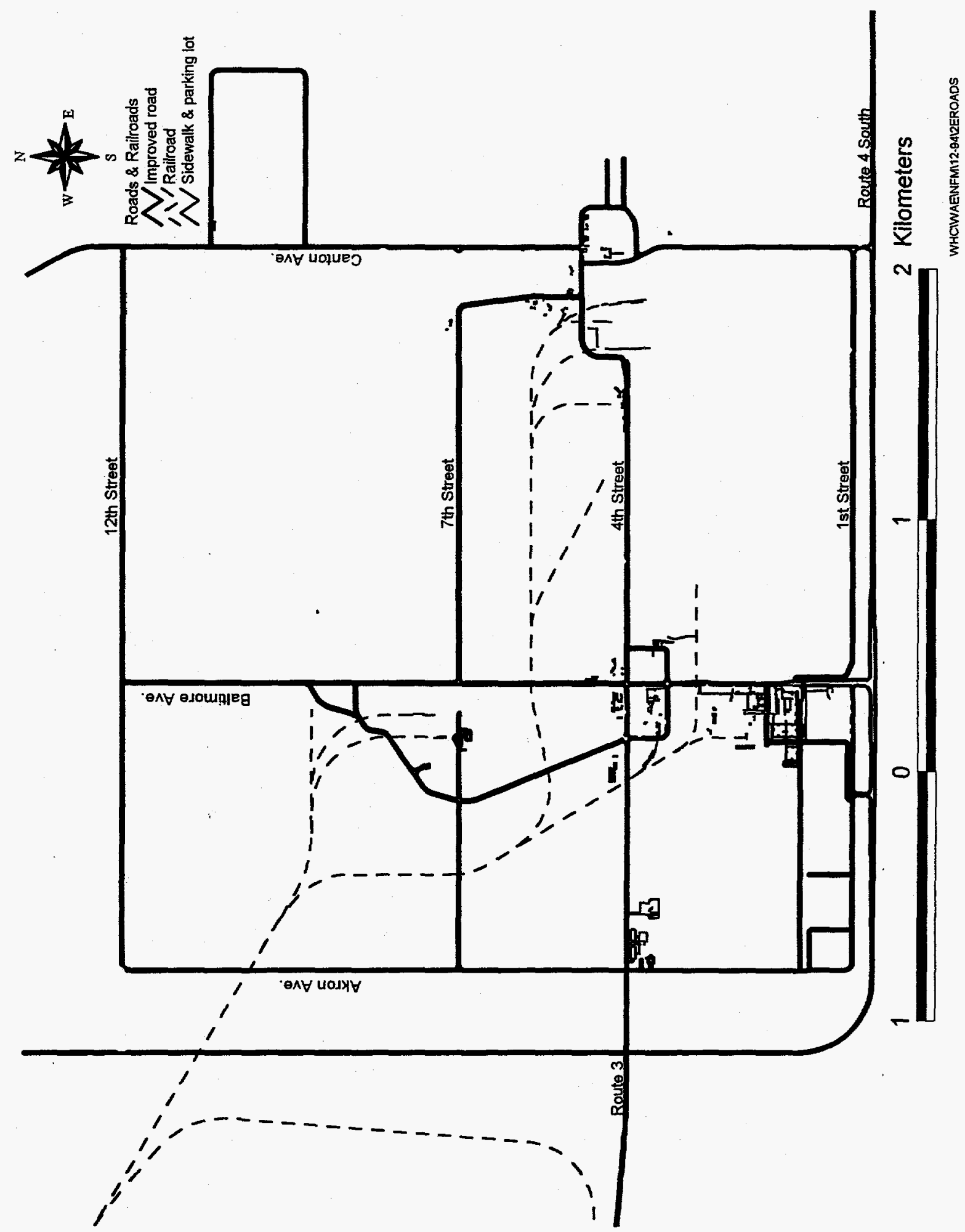


WHC-SP-0098-6

Figure A-25. 200 West Area Roads and Railways.

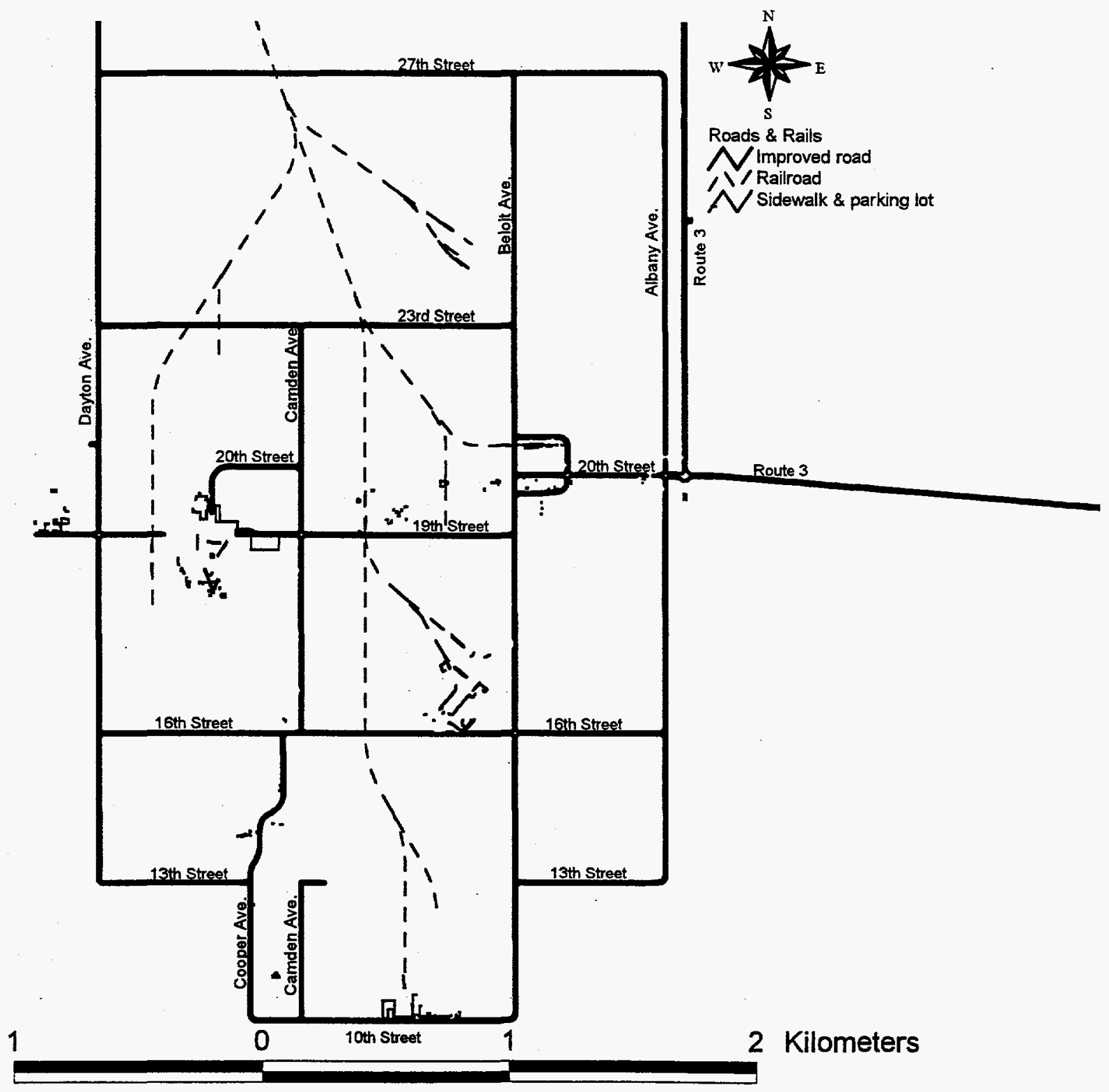


WHC-SP-0098-6

Figures A-26 through A-32 show air sample locations. 
Figure A-26. 100-N Ambient Air Sample Location.

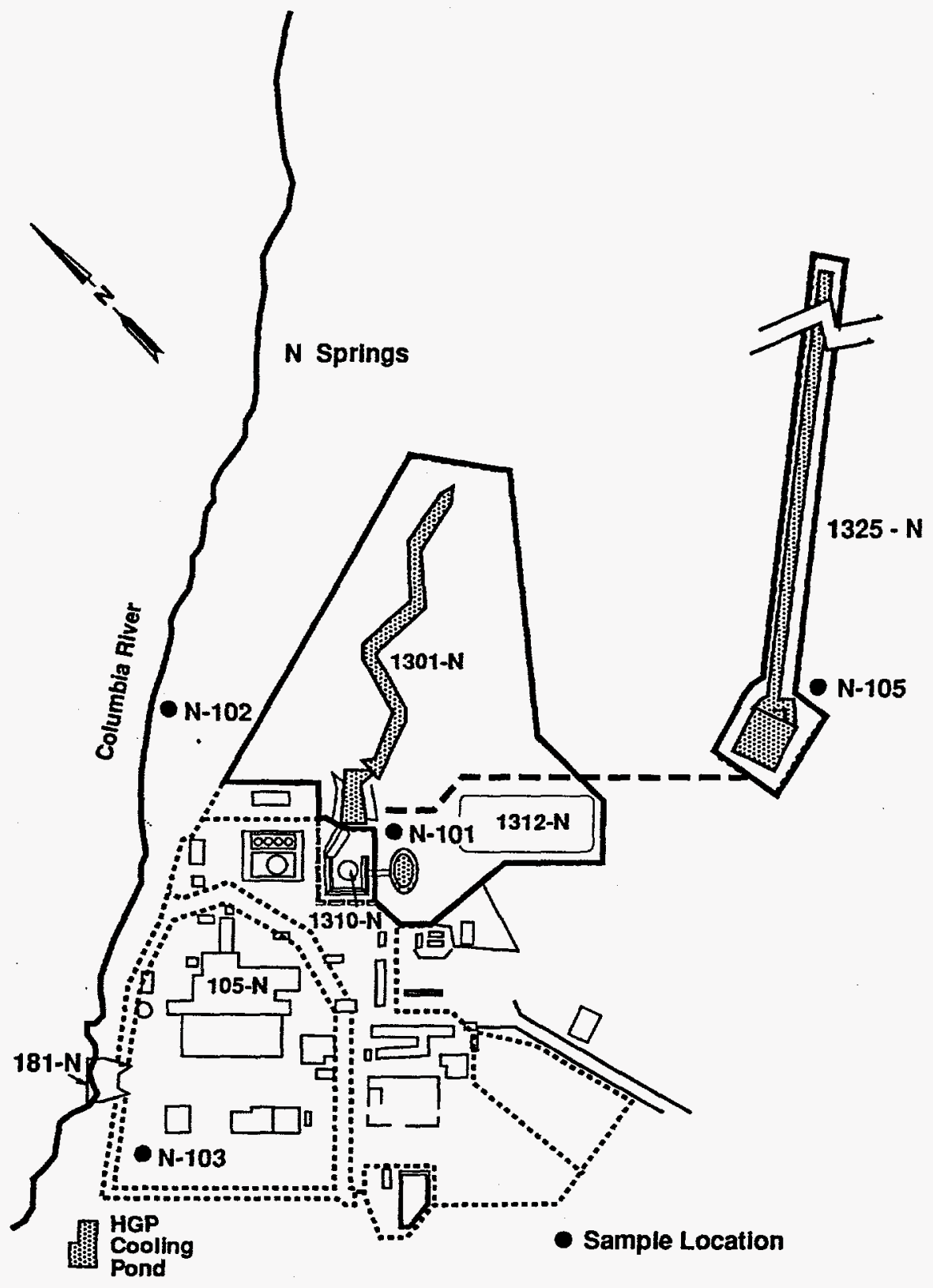

29204047.1 
Figure A-27. 100-K Ambient Air Sample Location.

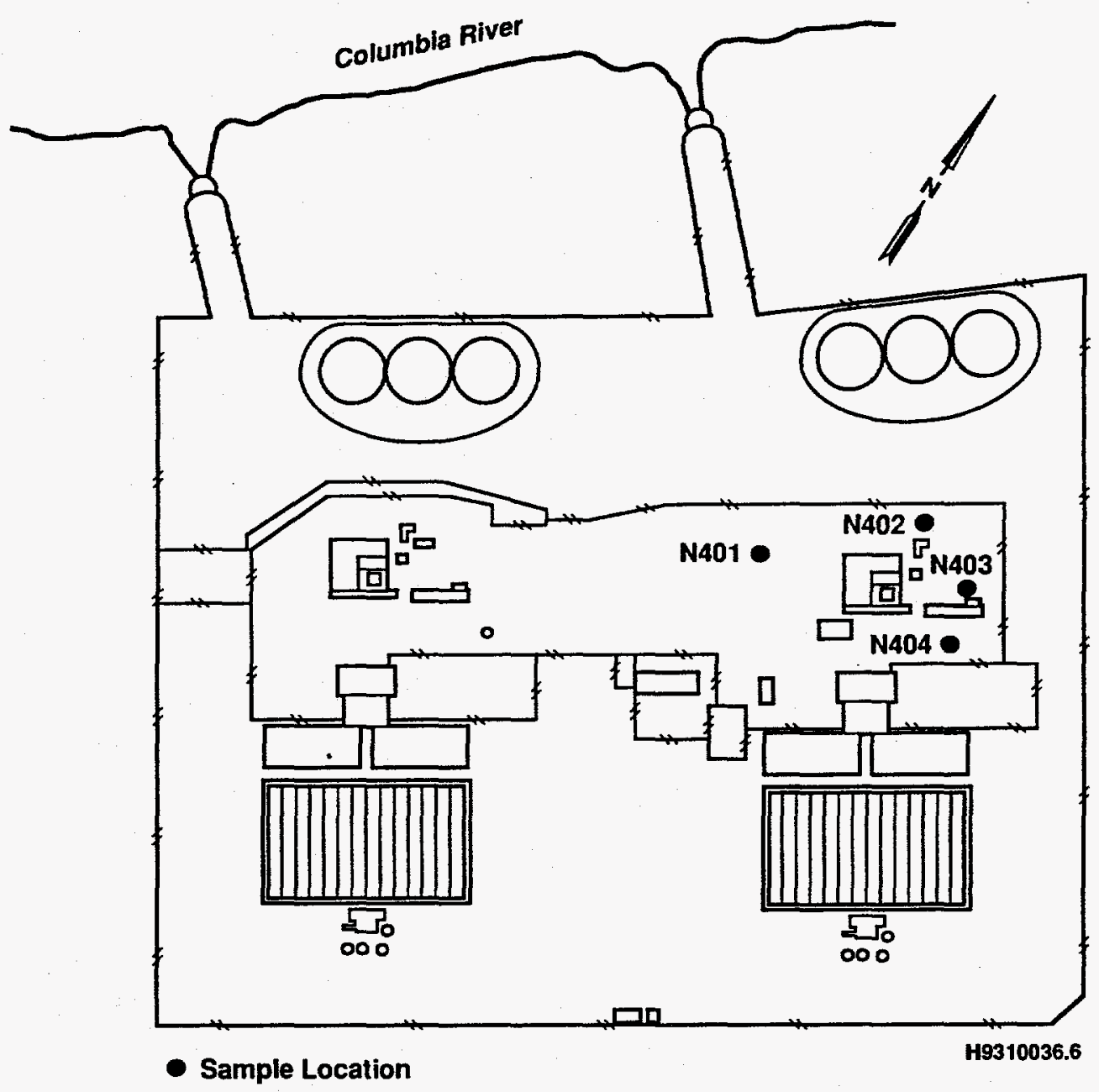


WHC-SP-0098-6

Figure A-28. 200 East Air Sample Locations.

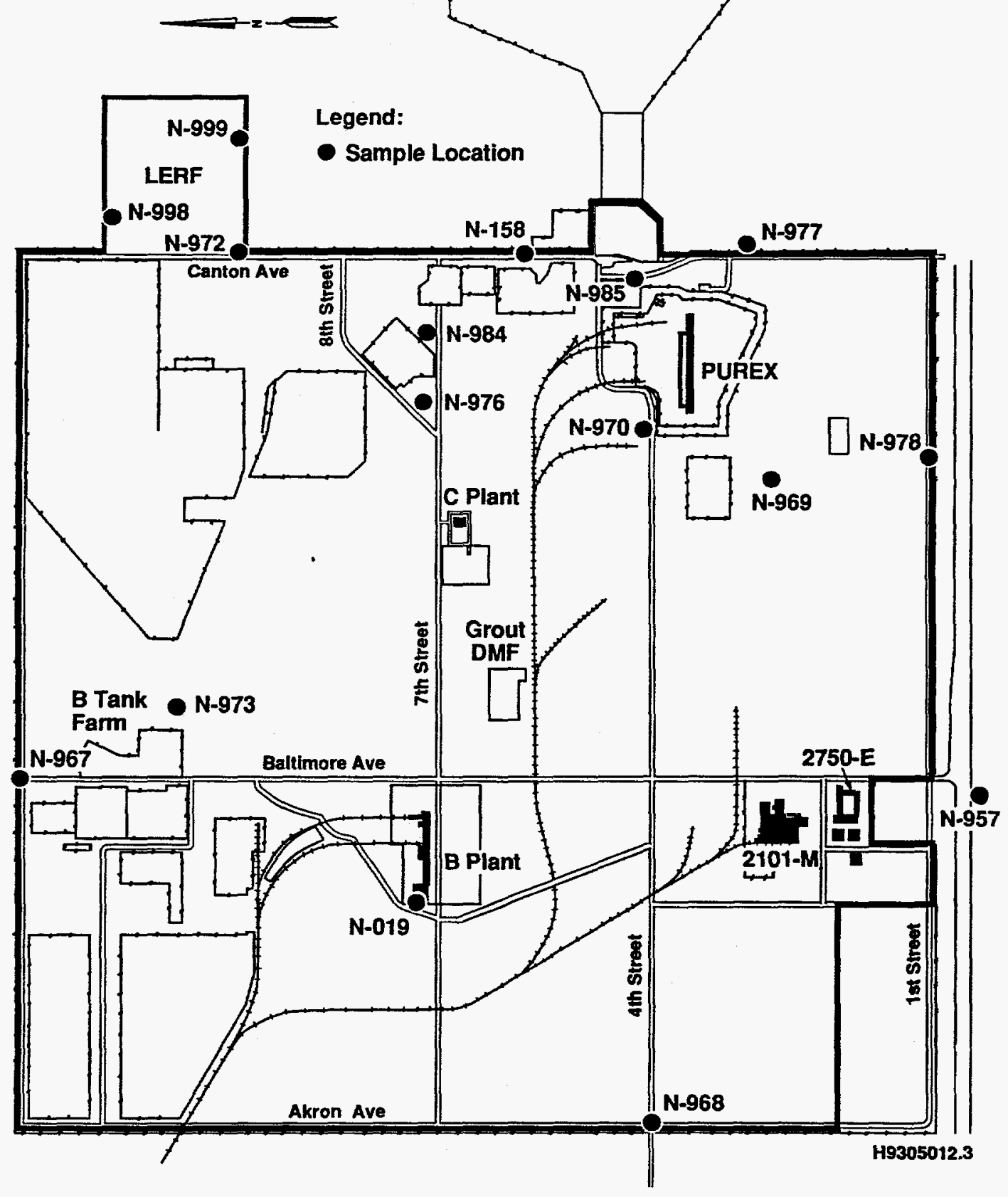


WHC-SP-0098-6

Figure A-29. 200 West Air Sample Locations.

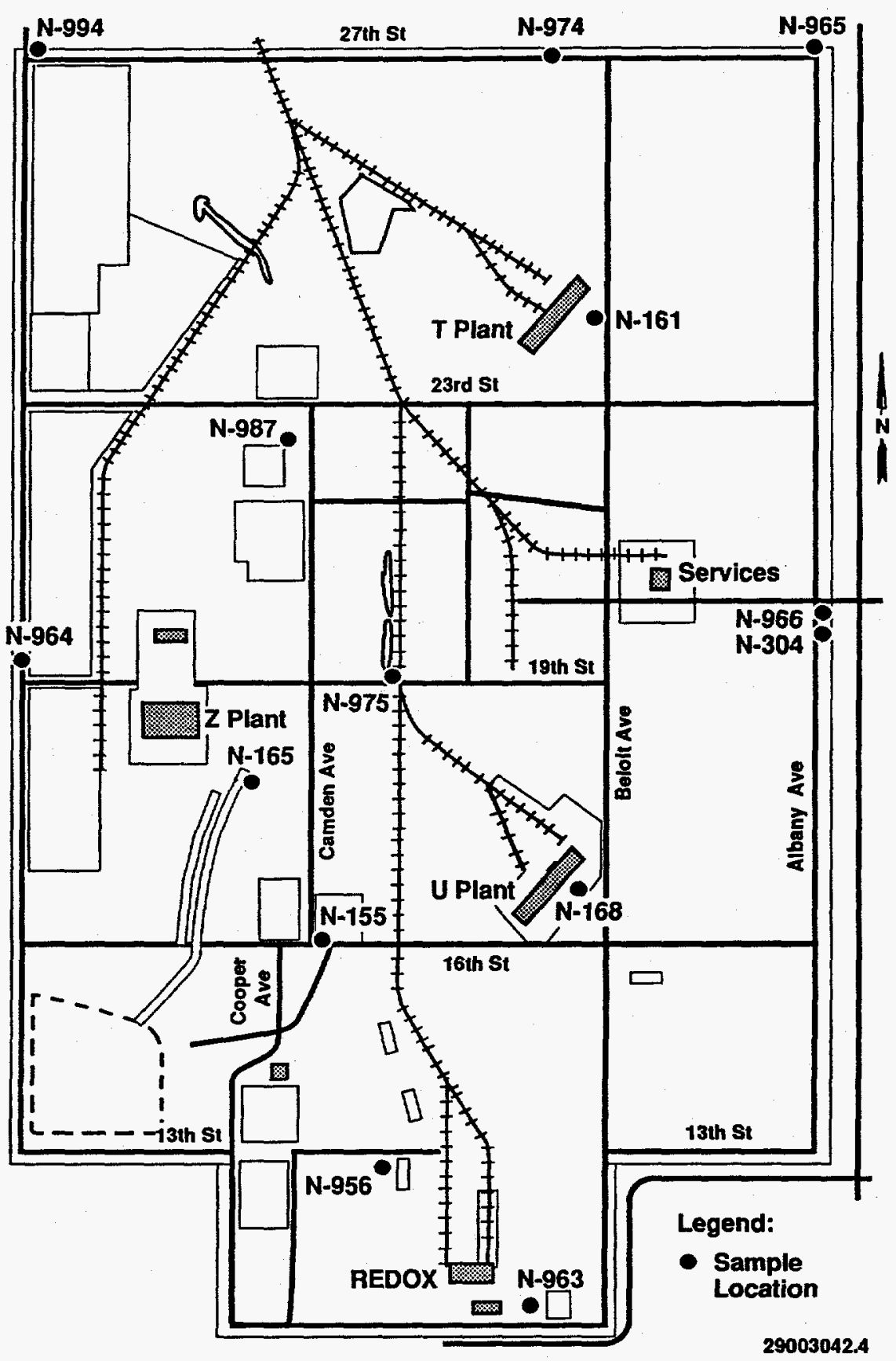


WHC-SP-0098-6

Figure A-30. 200 East/West Vent Station Air Sample Locations.

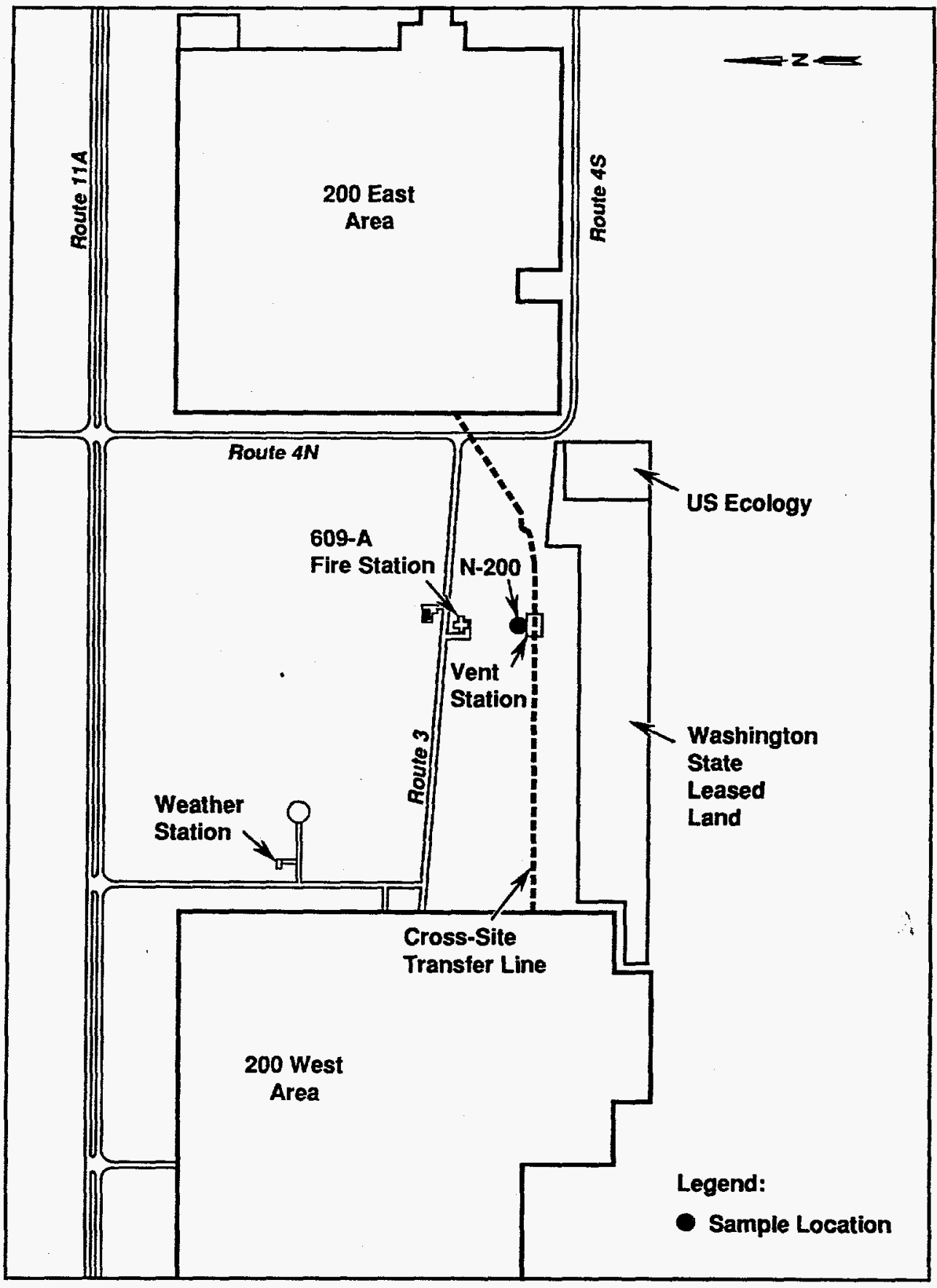

H9306026.1a 
Figure A-31. 600 Area Air Sample Locations.

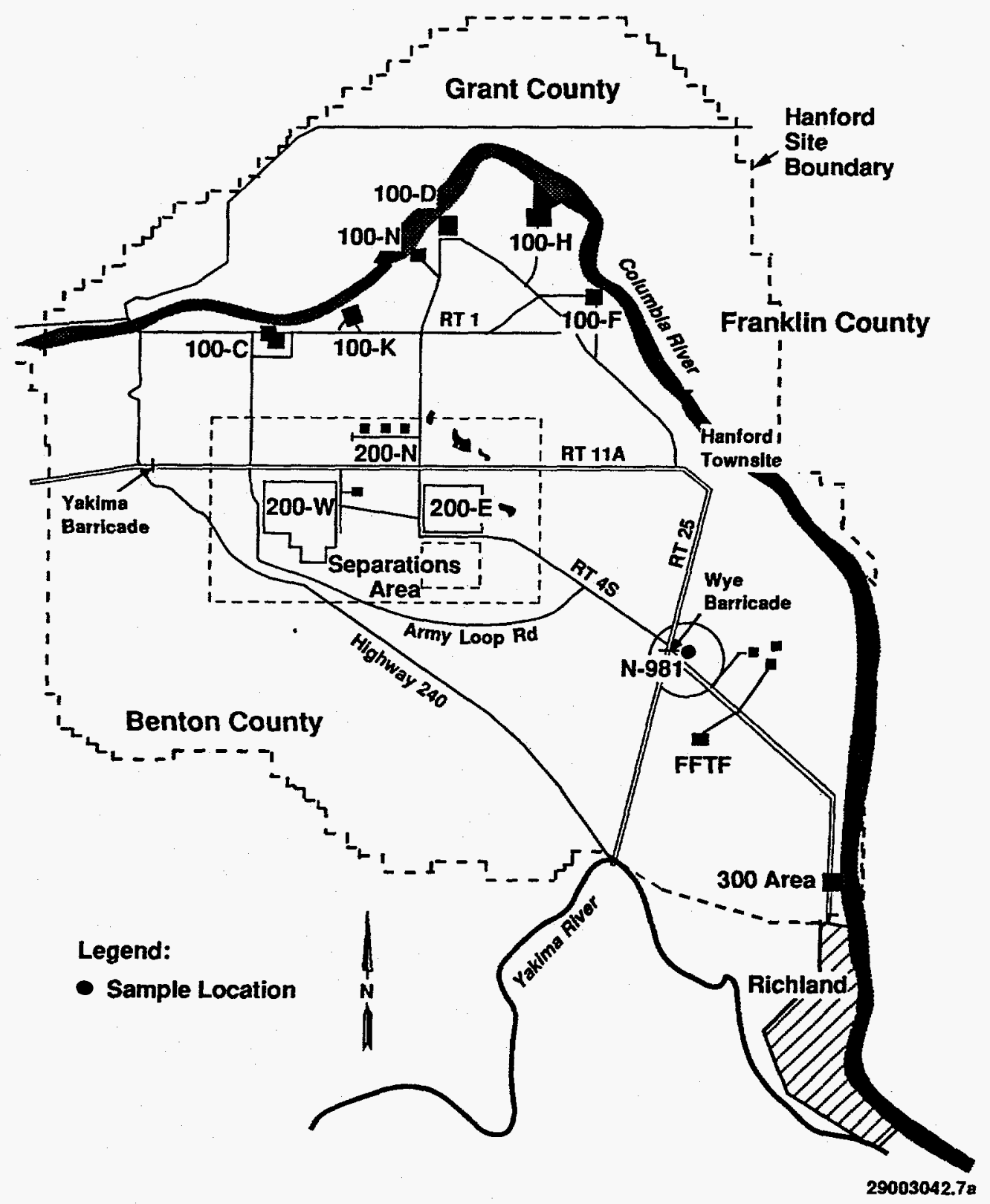


WHC-SP-0098-6

Figure A-32. 300 Area Ambient Air Sampler Locations.

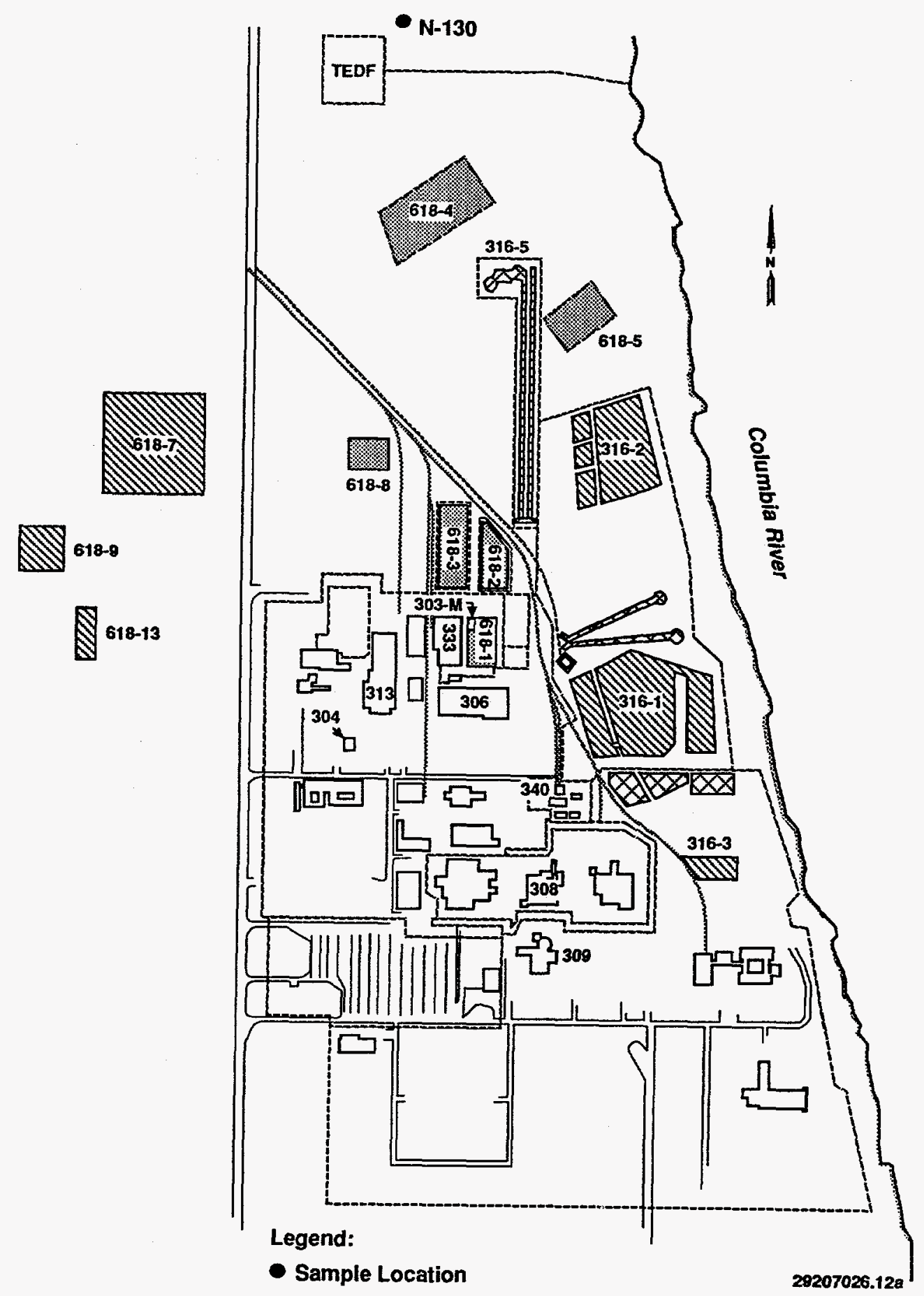


WHC-SP-0098-6

Figures A-33 through A-45 show TLD and dose rate locations. 
WHC-SP-0098-6

Figure A-33. 100-N Area TLD Locations.

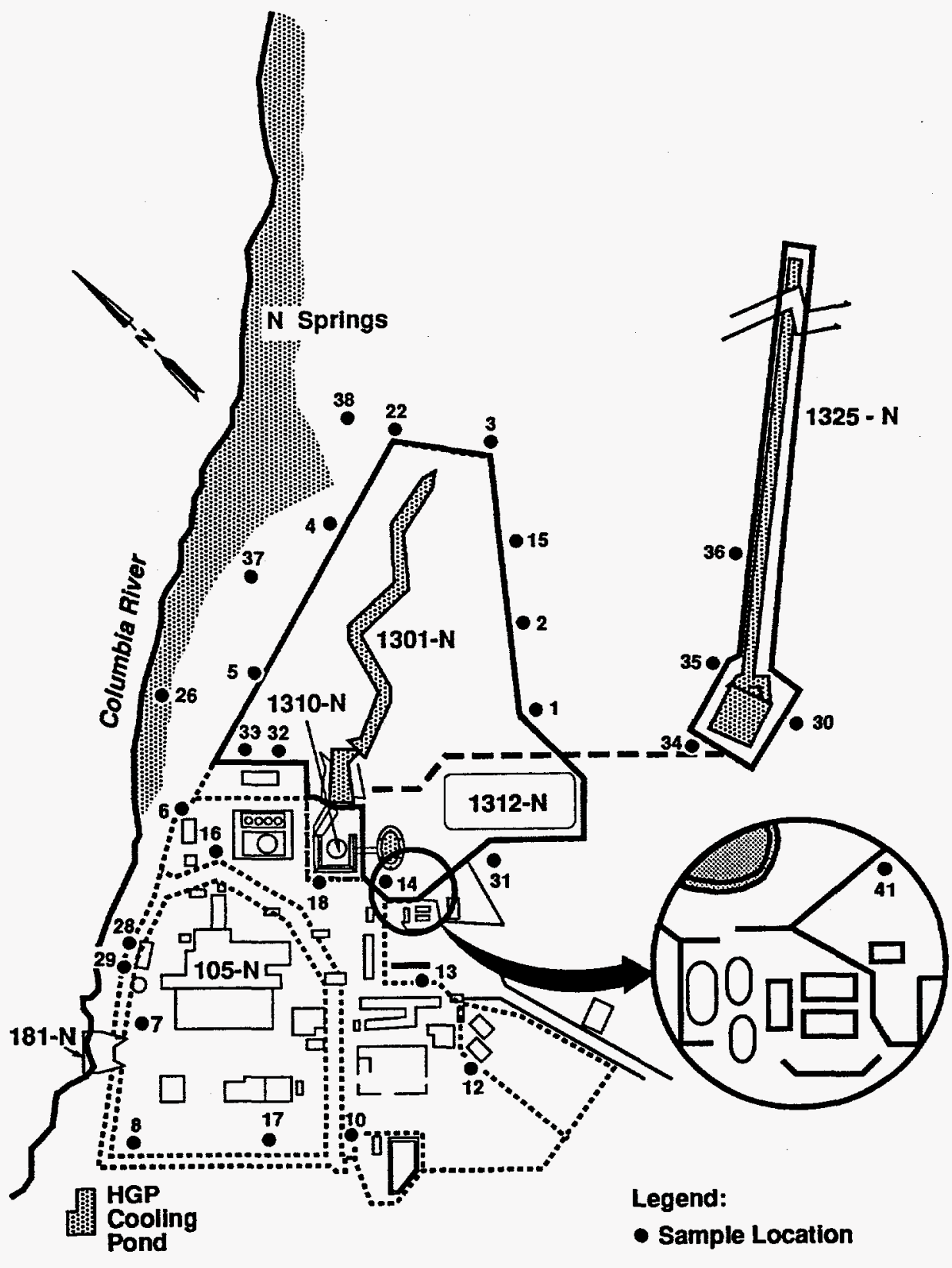

$29202060.1 A$ 
WHC-SP-0098-6

Figure A-34. N Springs Dose Rate Locations.

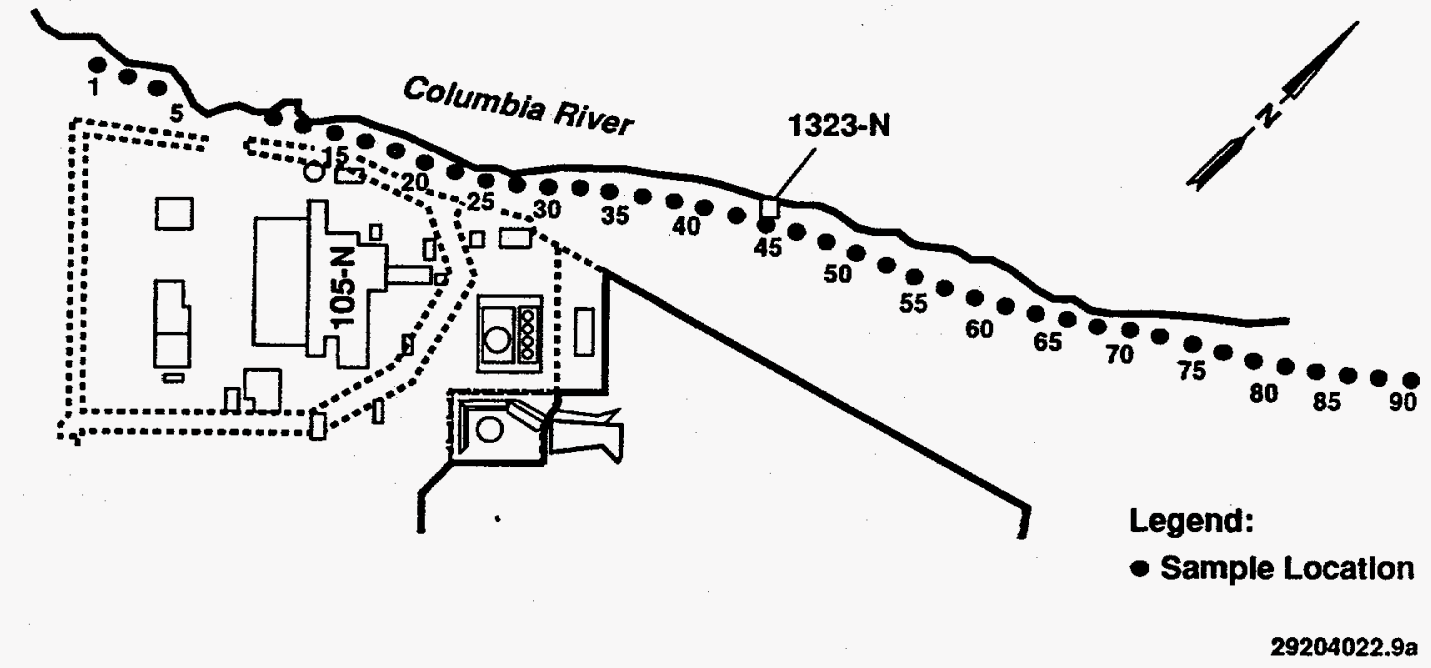


Figure A-35. 1301-N Dose Rate Locations.

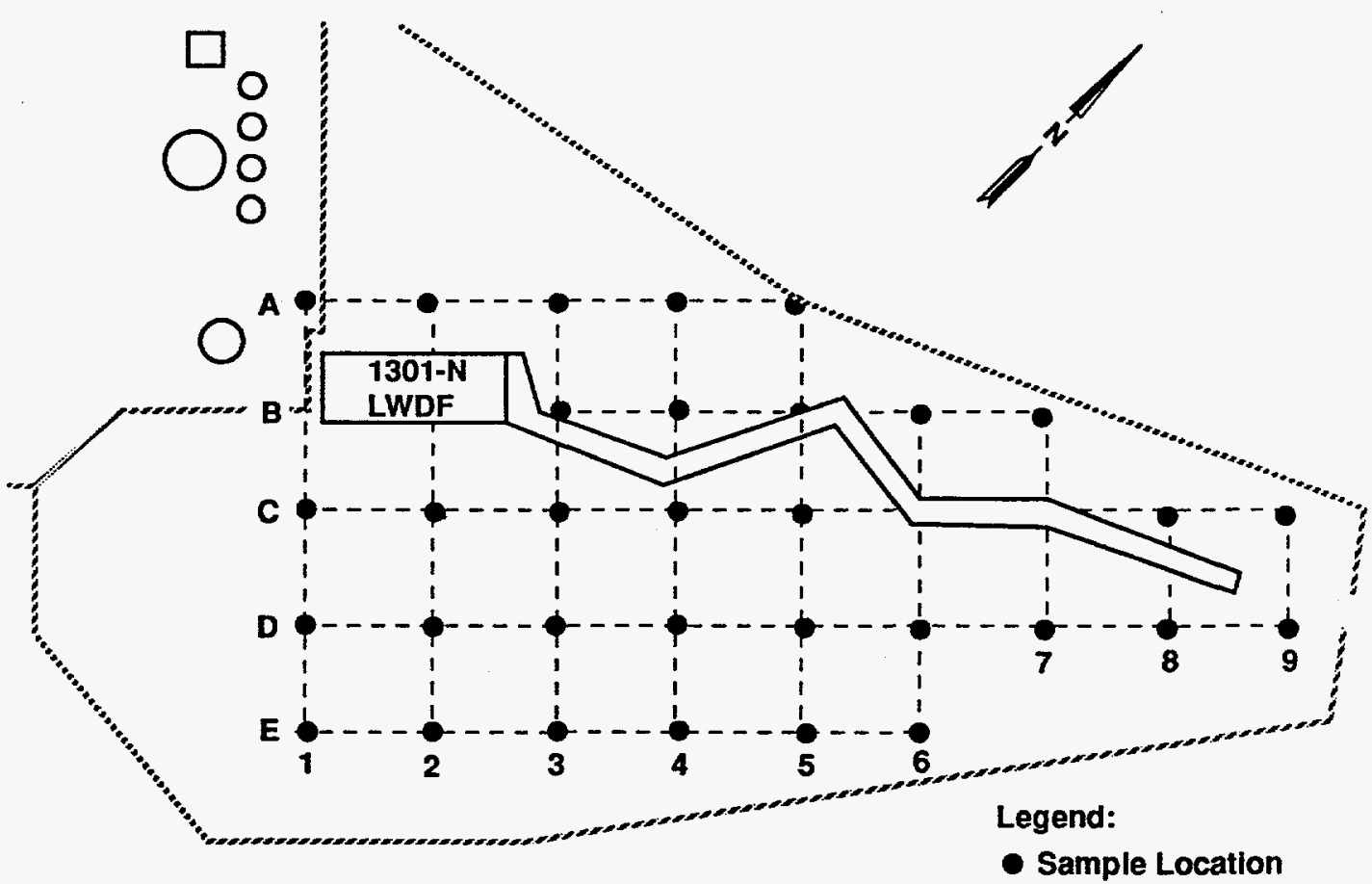

29204022.10a 
Figure A-36. 1325-N Dose Rate Locations.

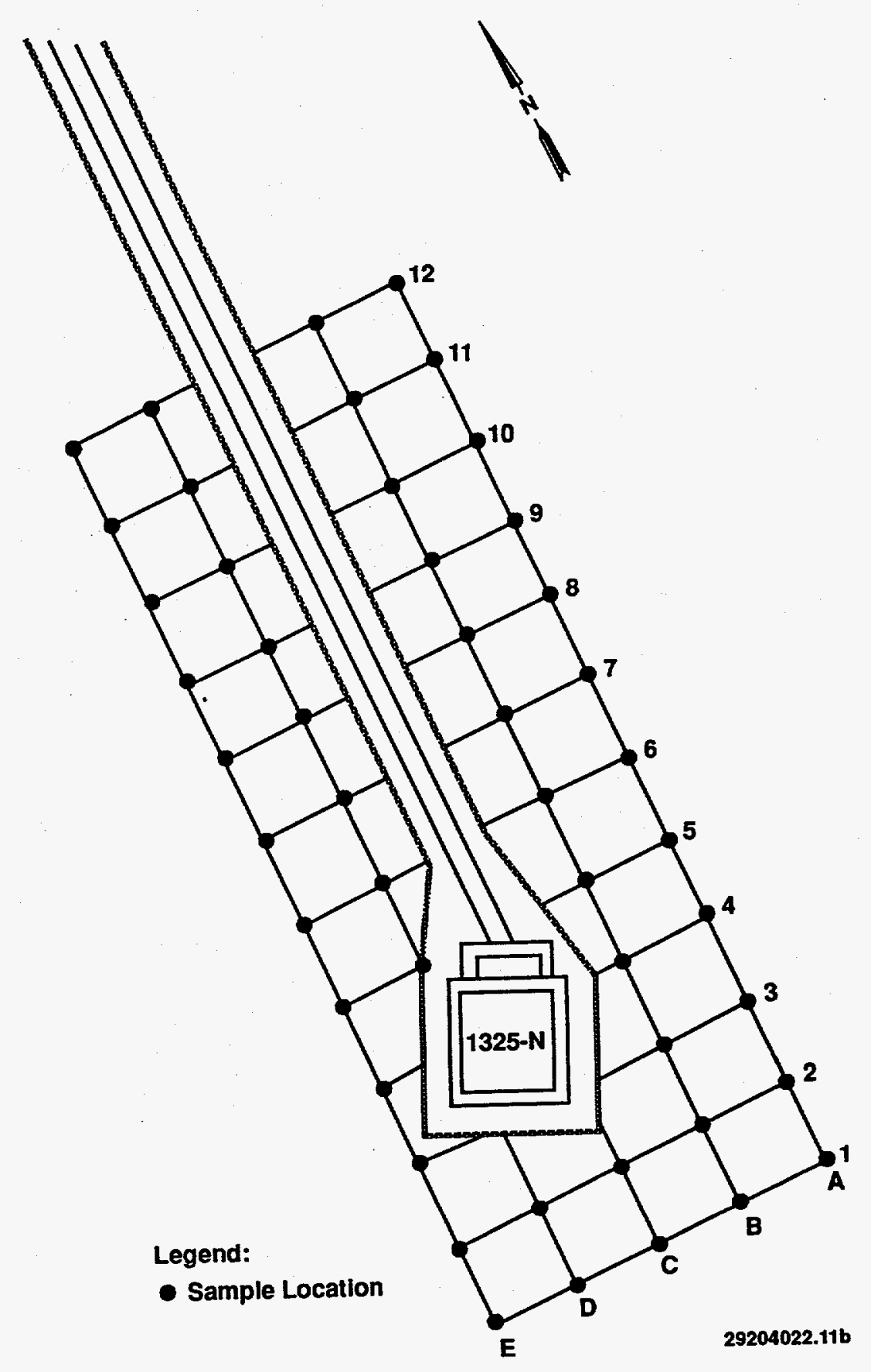


WHC-SP-0098-6

Figure A-37. 100-K Area TLD Locations.

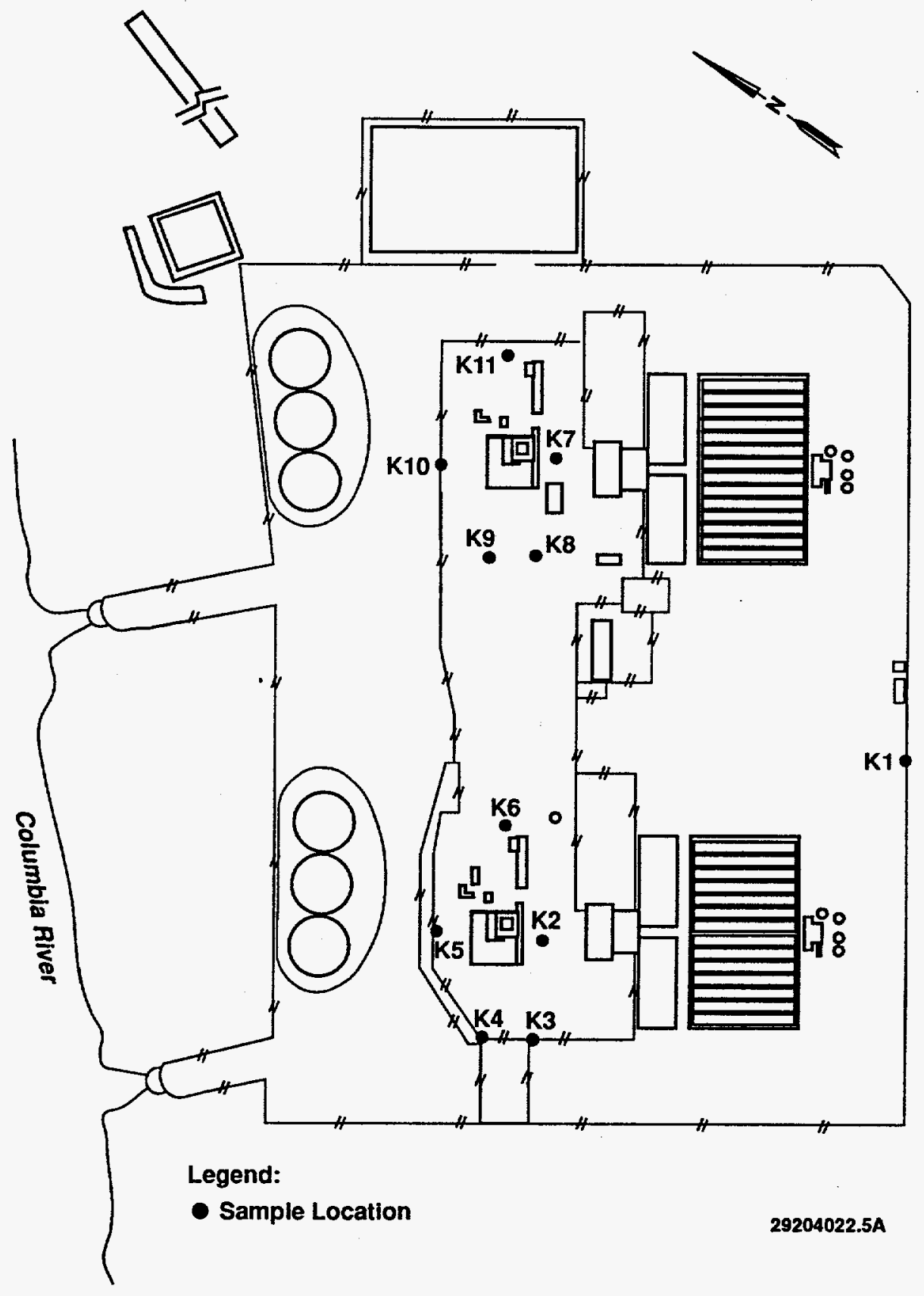


Figure A-38. 200 East TLD Locations.

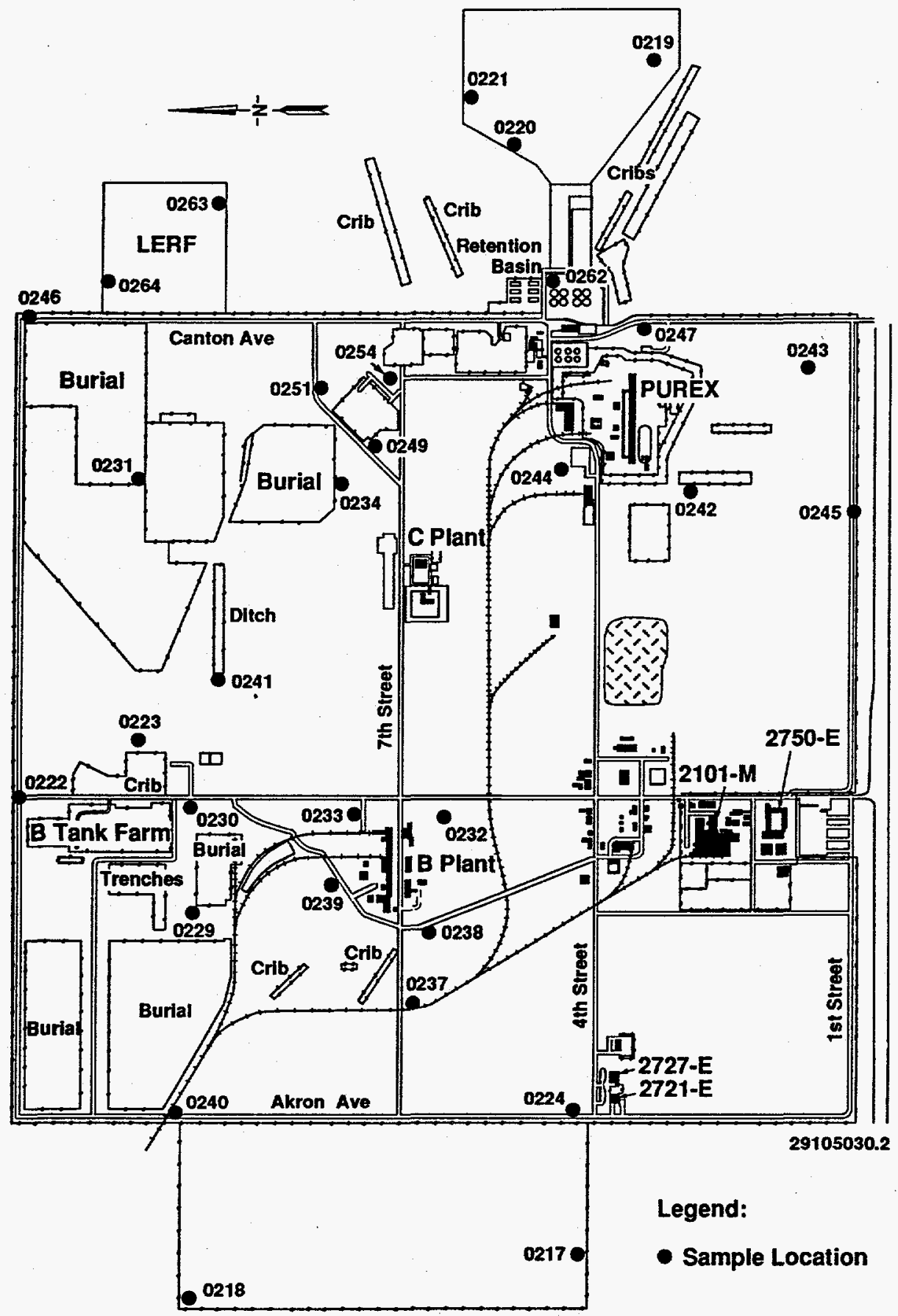


WHC-SP-0098-6

Figure A-39. 200 East A-Tank Farm TLD Locations.

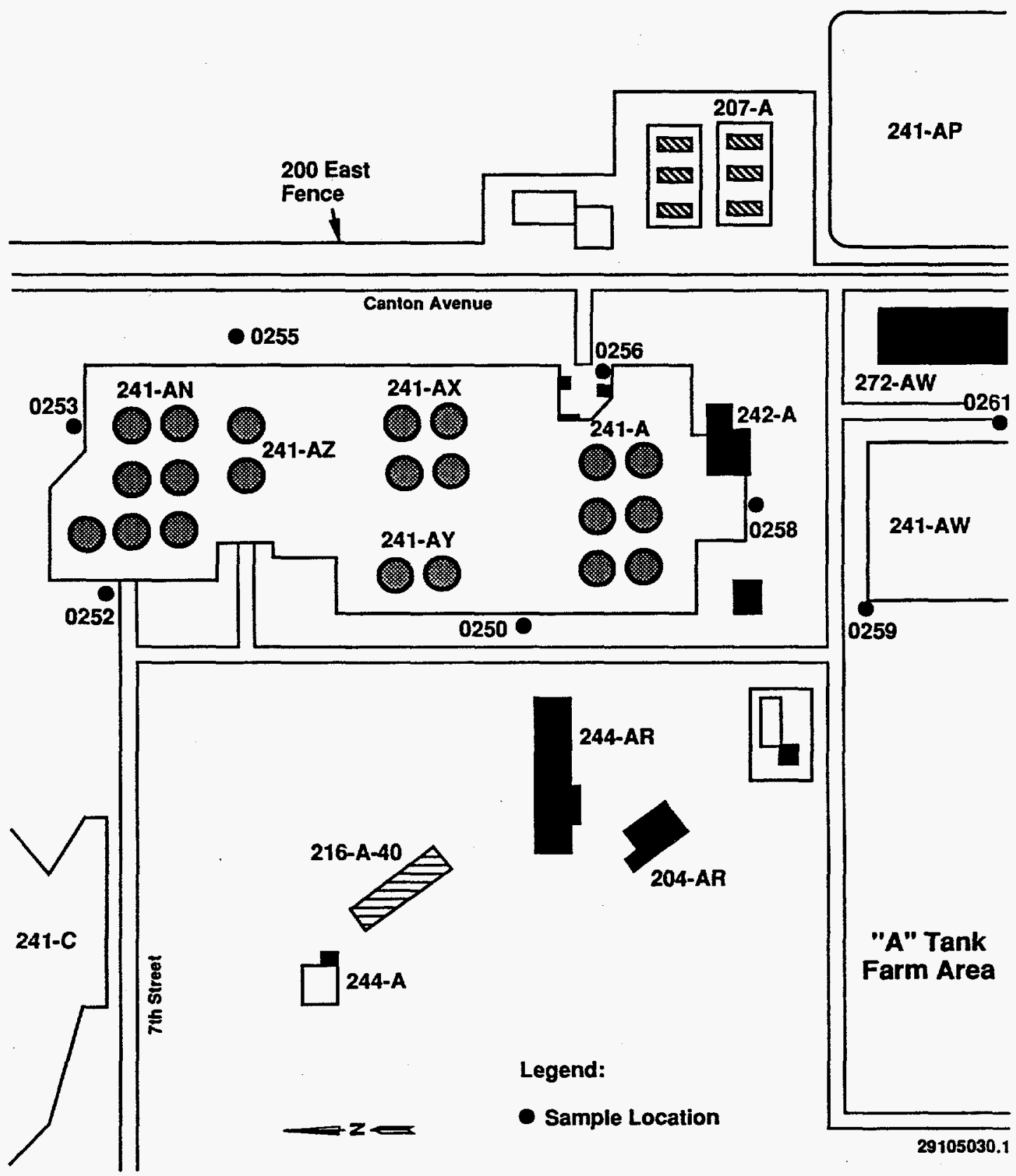


Figure A-40. 200 West TLD Locations.

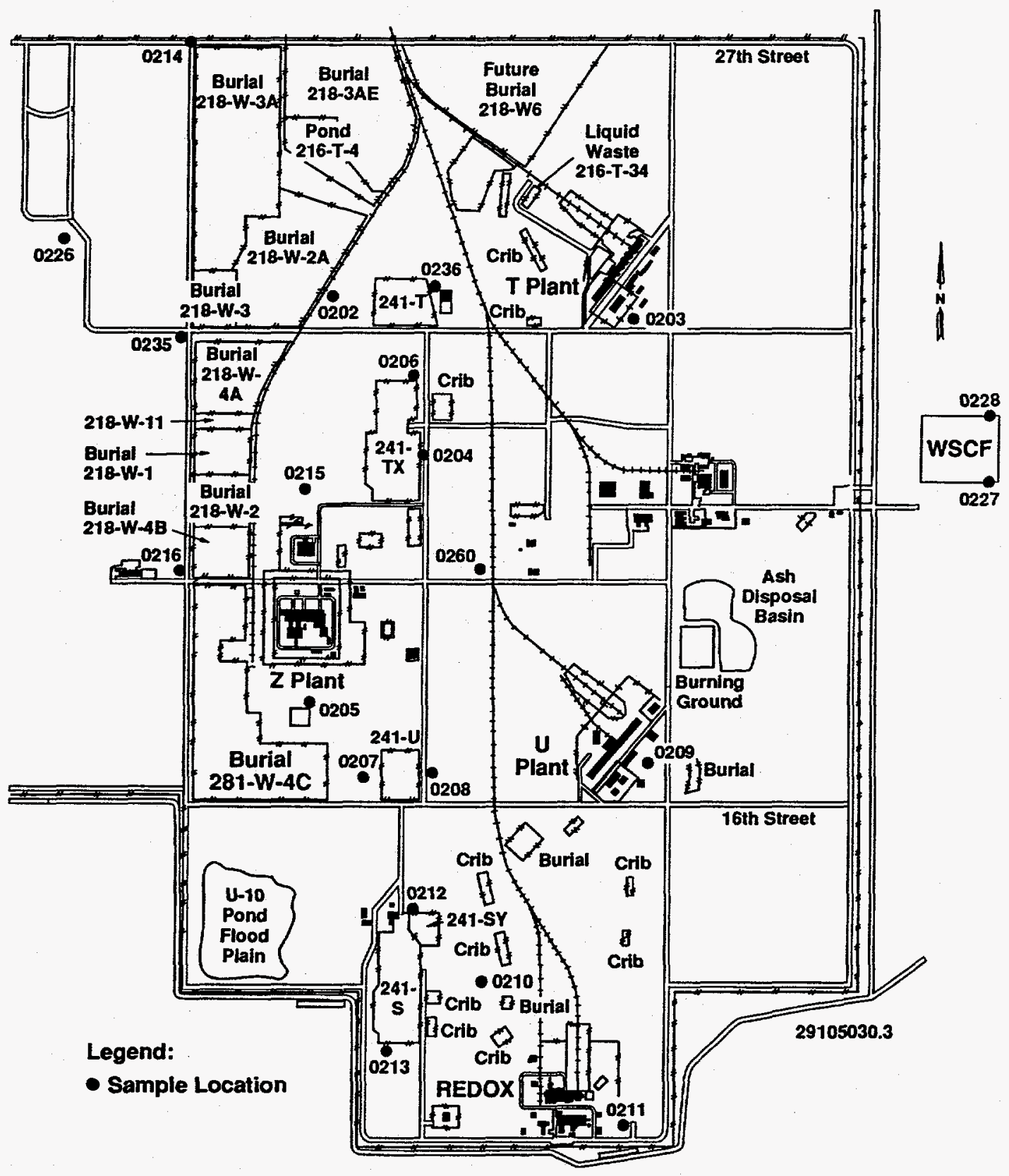


Figure A-41. 200 West Area Dose Rate and Contamination Survey Plot Locations.

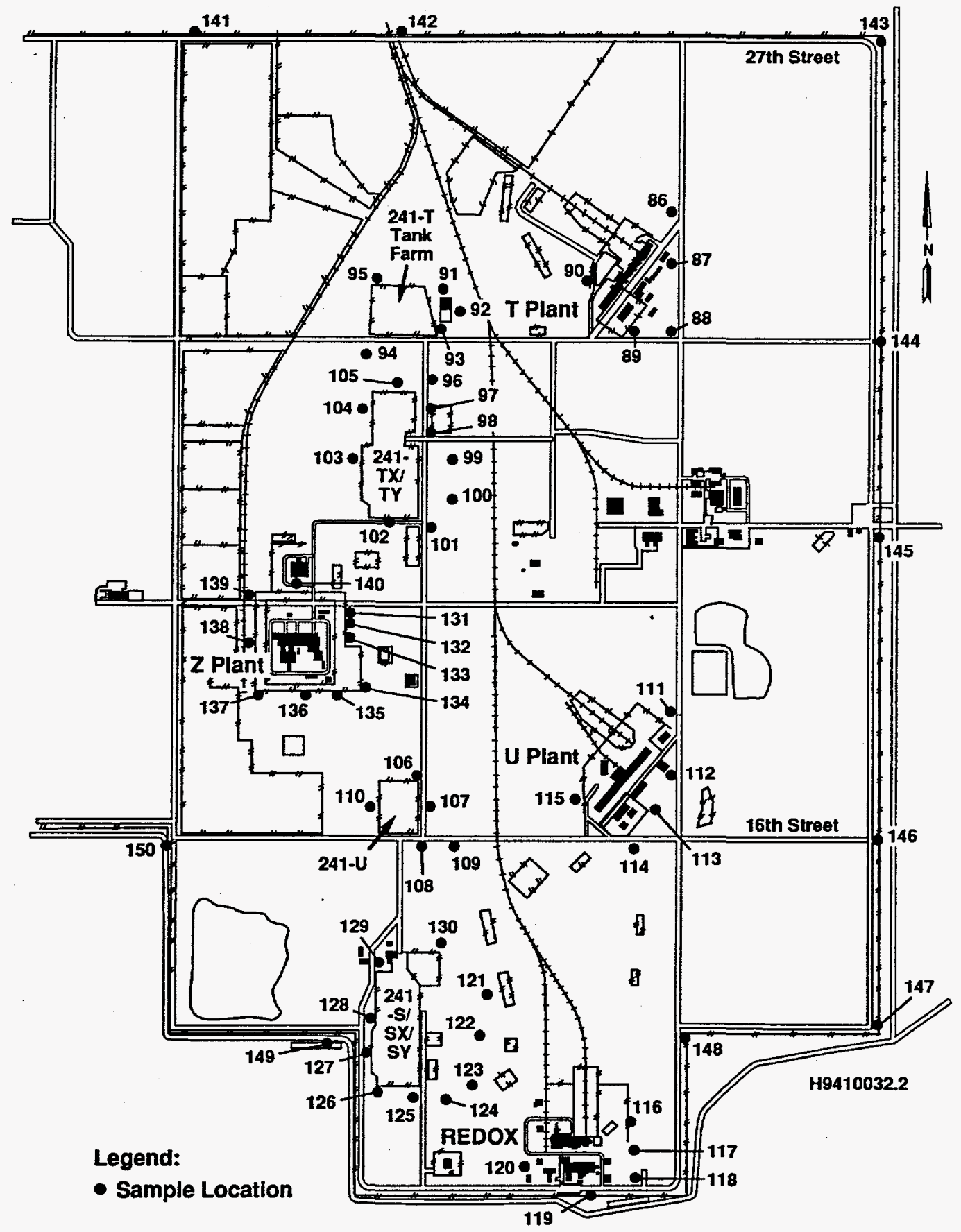


Figure A-42. 200 East Area Dose Rate and Contamination Survey Plot Locations.

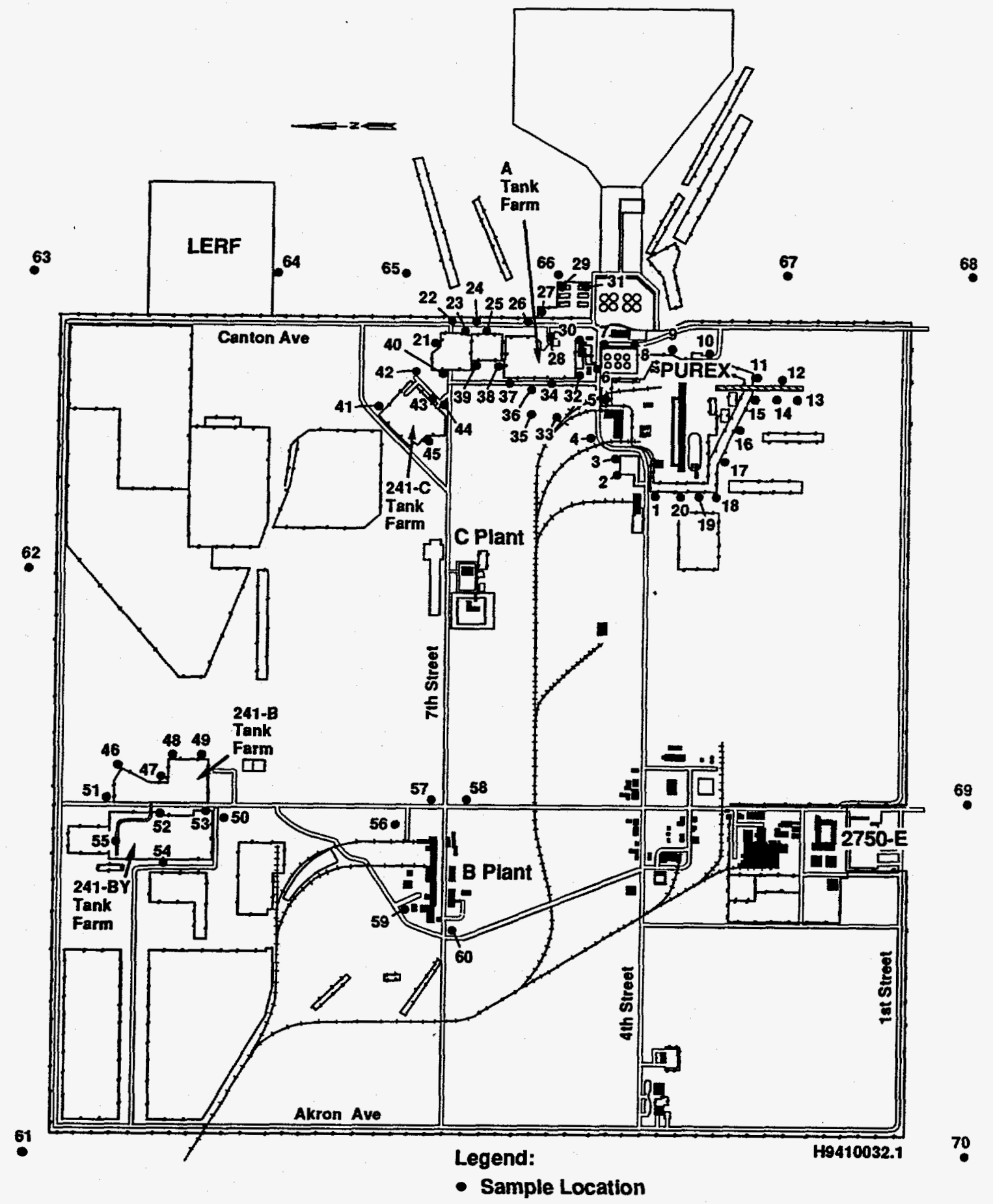


WHC-SP-0098-6

Figure A-43. 300 Area TLD Locations.

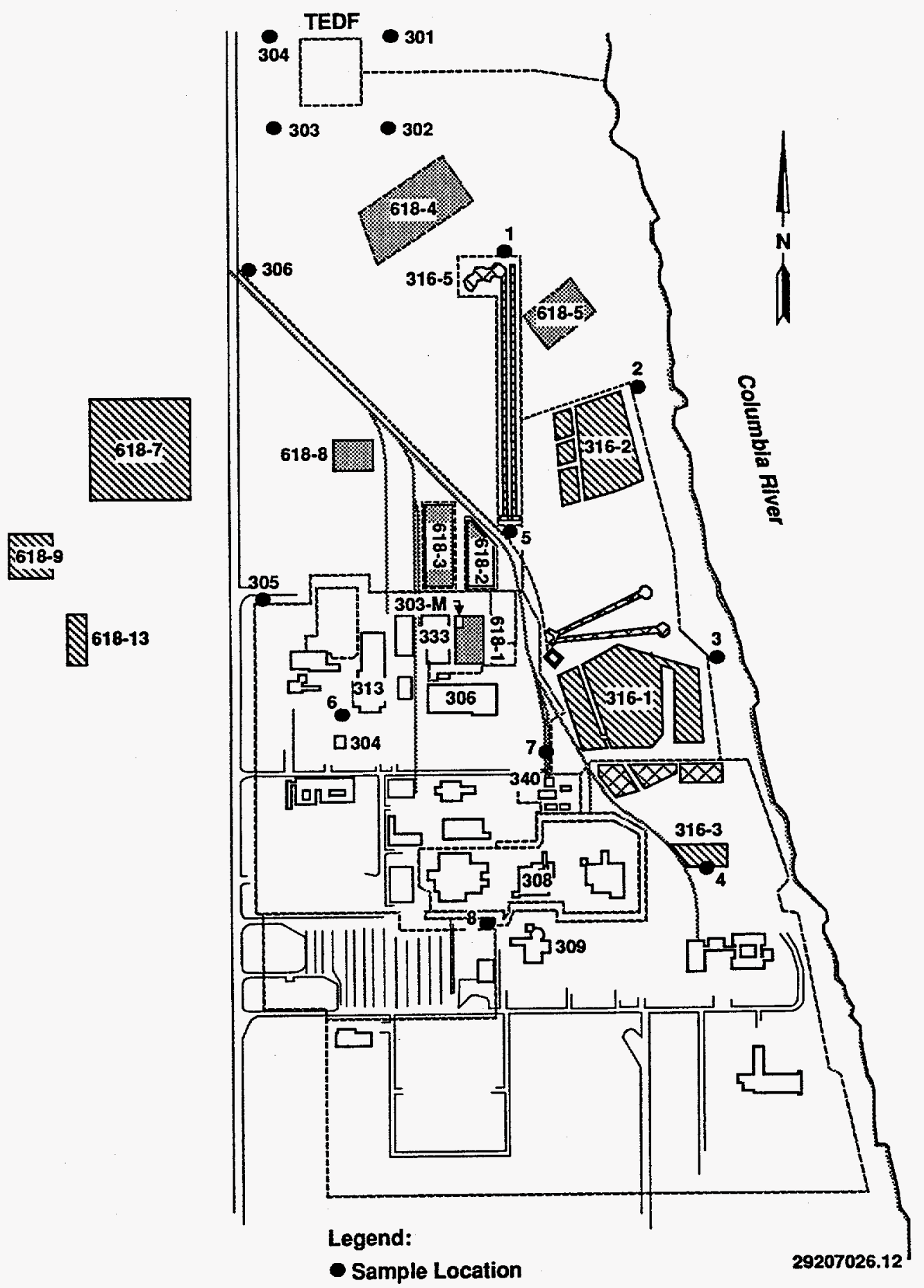


Figure A-44. 400 Area TLD Locations.

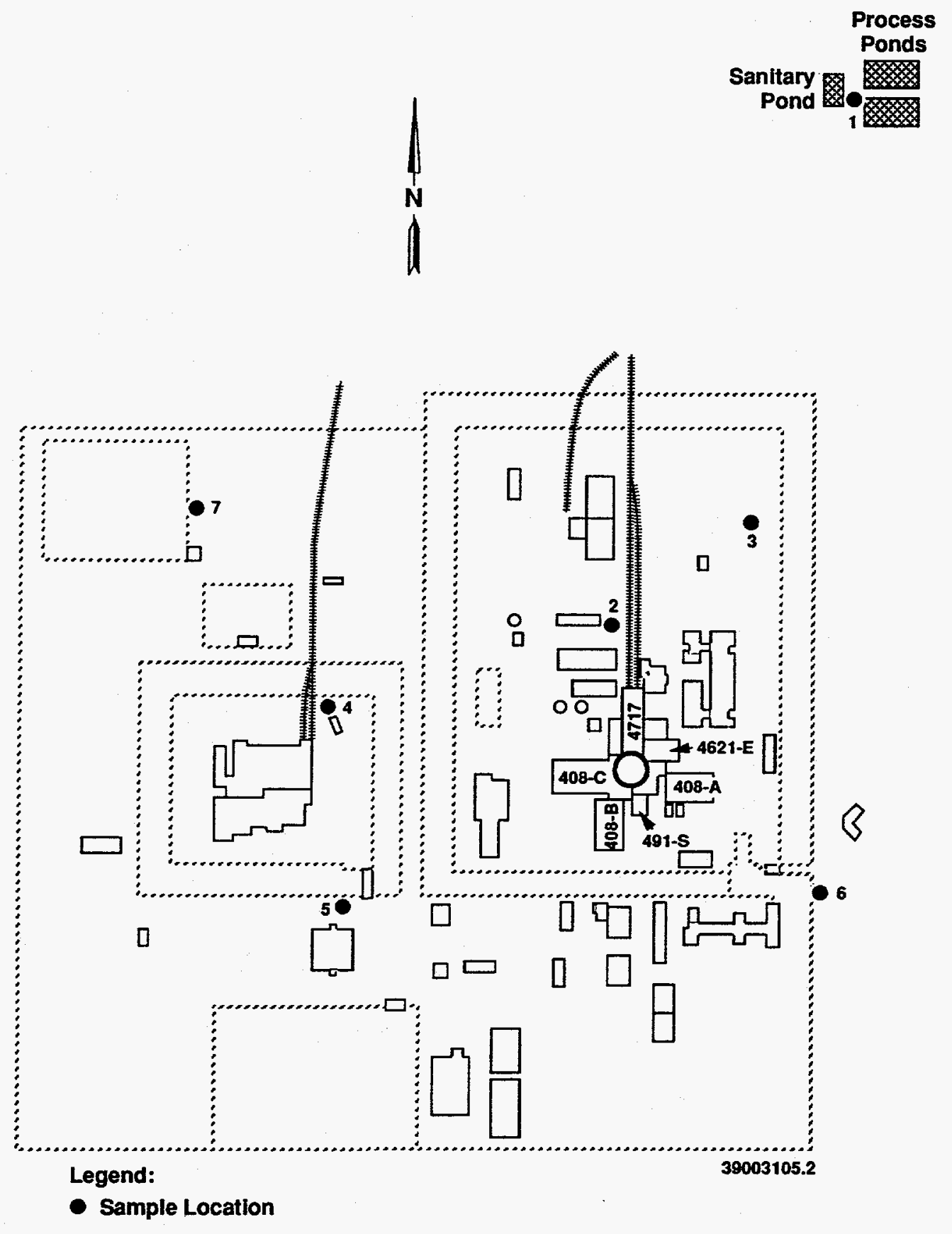


WHC-SP-0098-6

This page intentionally left blank. 
WHC-SP-0098-6

Figures A-45 through A-51 show soil and vegetation sample locations. 
Figure A-45. 100-N Vegetation Sample Locations.

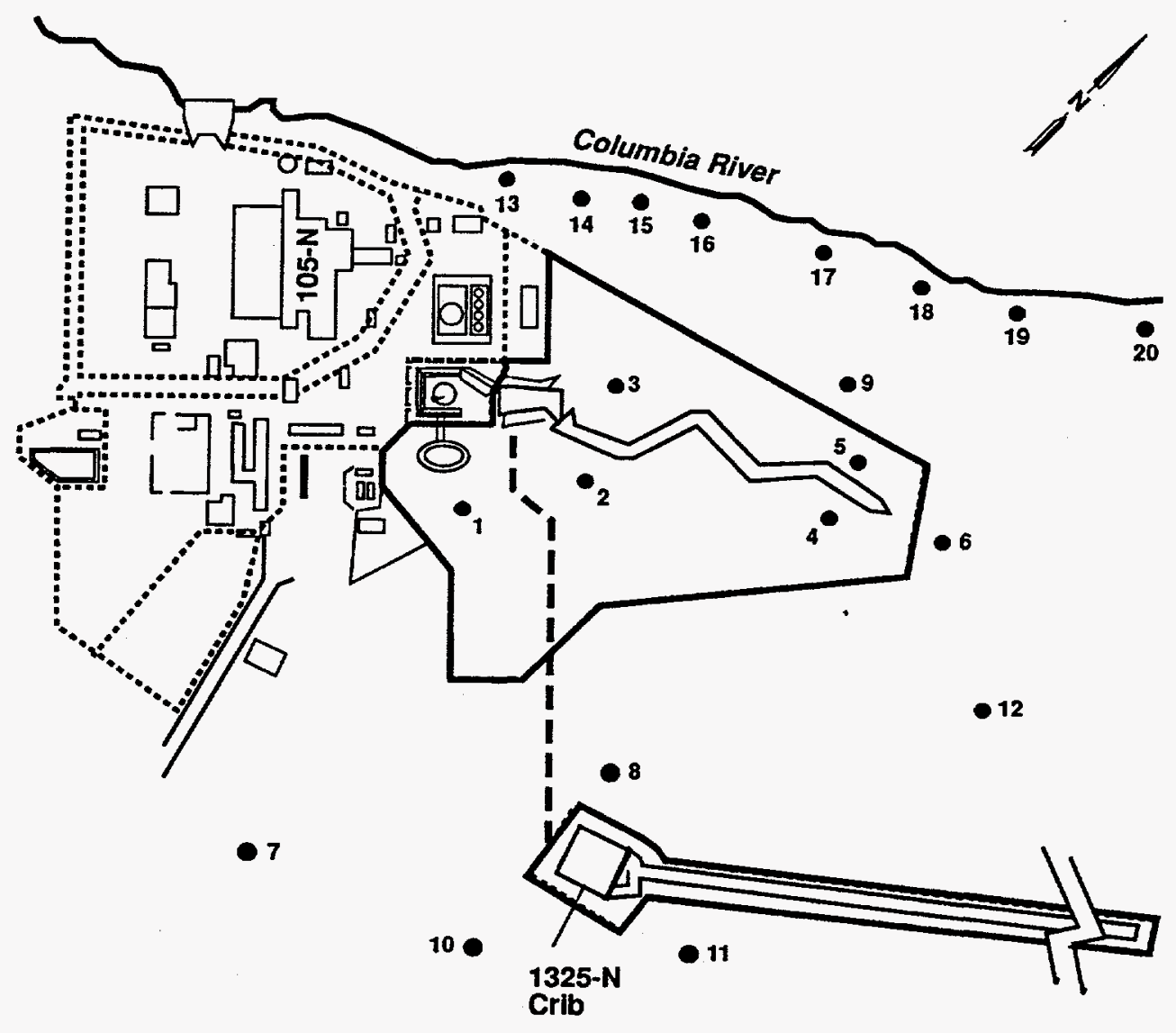

Legend:

- Sample Location

29204022.24 
Figure A-46. 100-N Soil Sample Locations.

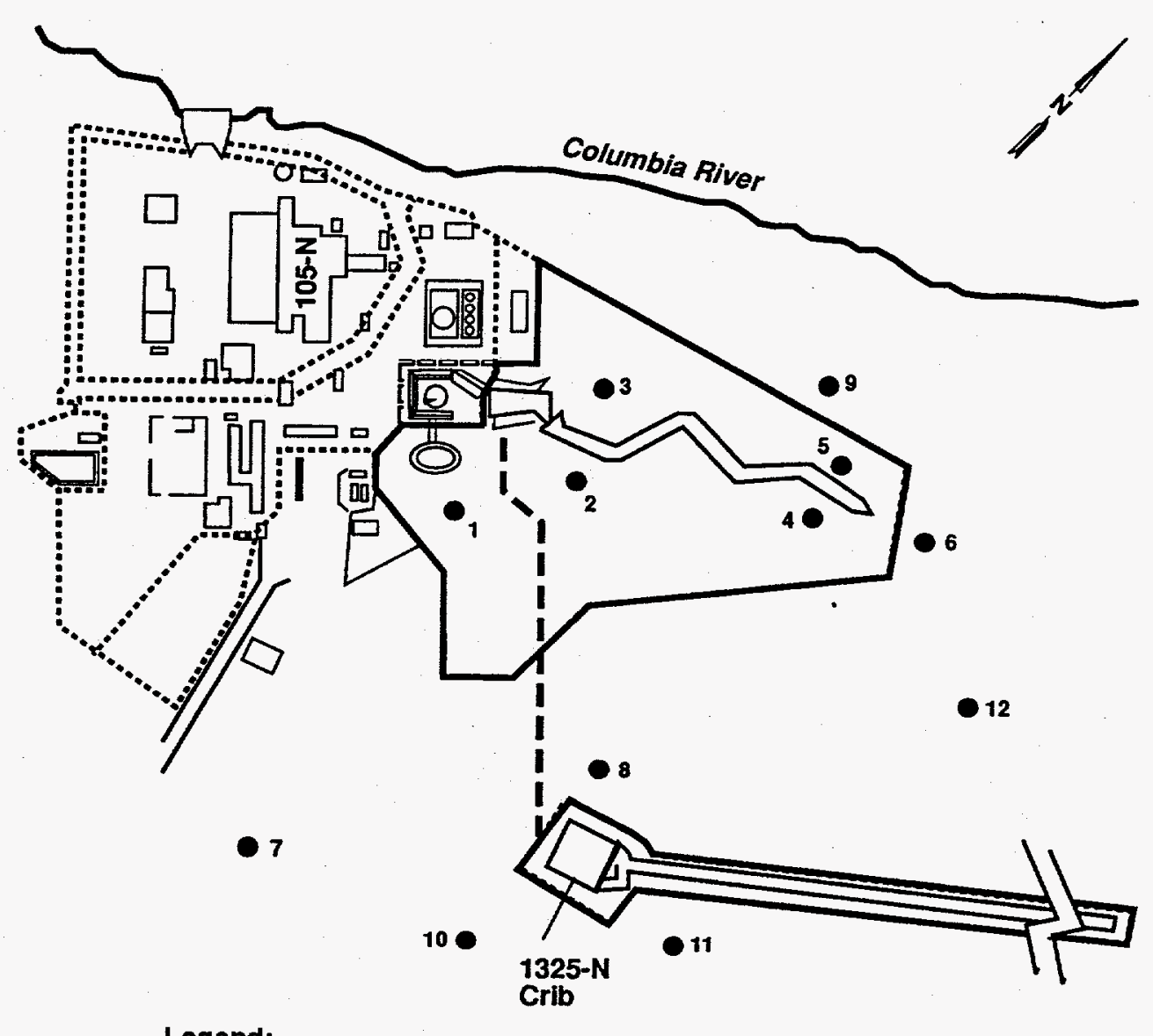

Legend:

- Sample Location

29204022.24a 
Figure A-47. 200 East Soil and Vegetation Sample Locations.

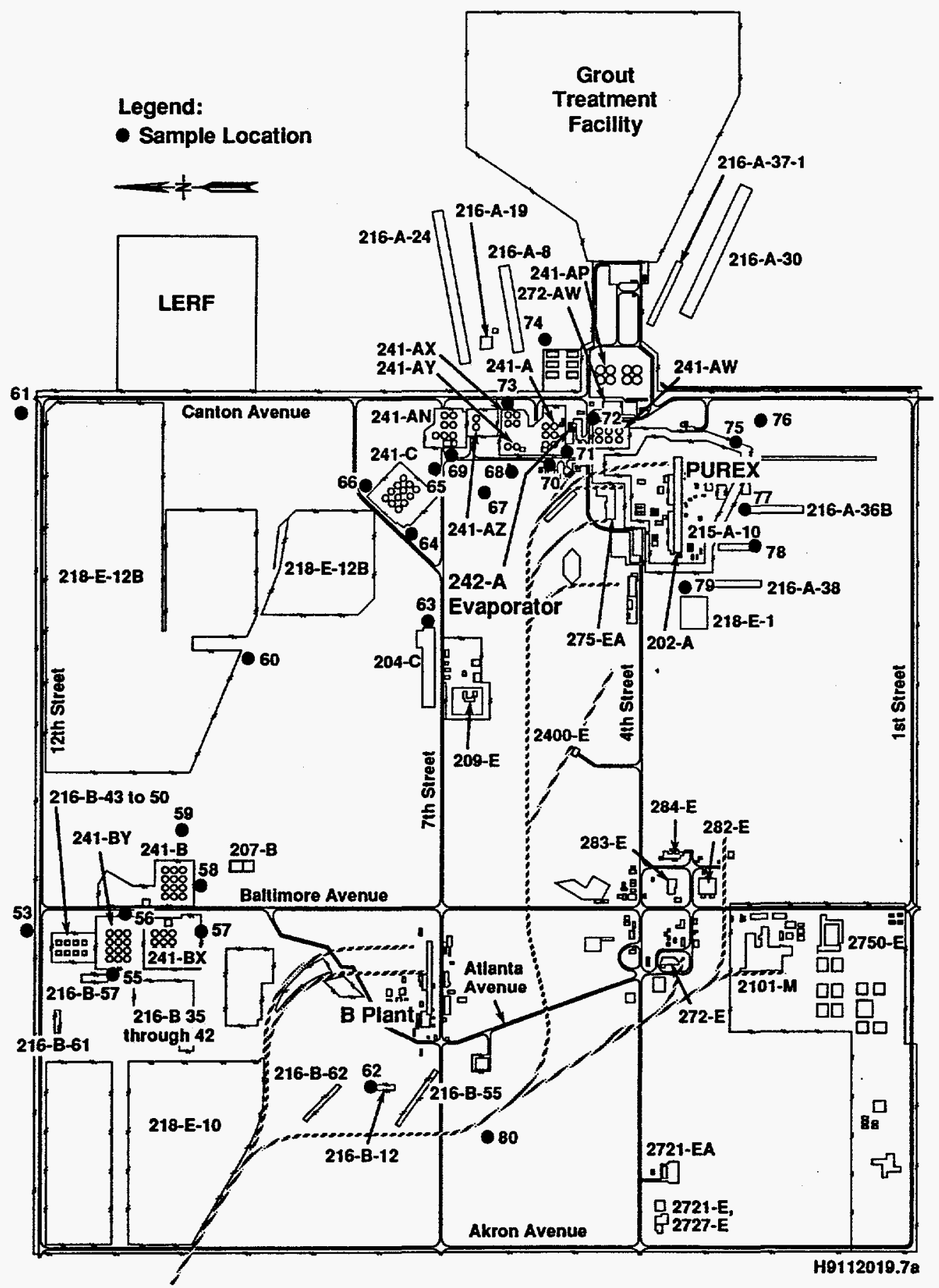


Figure A-48. 200 West Soil and Vegetation Sample Locations.

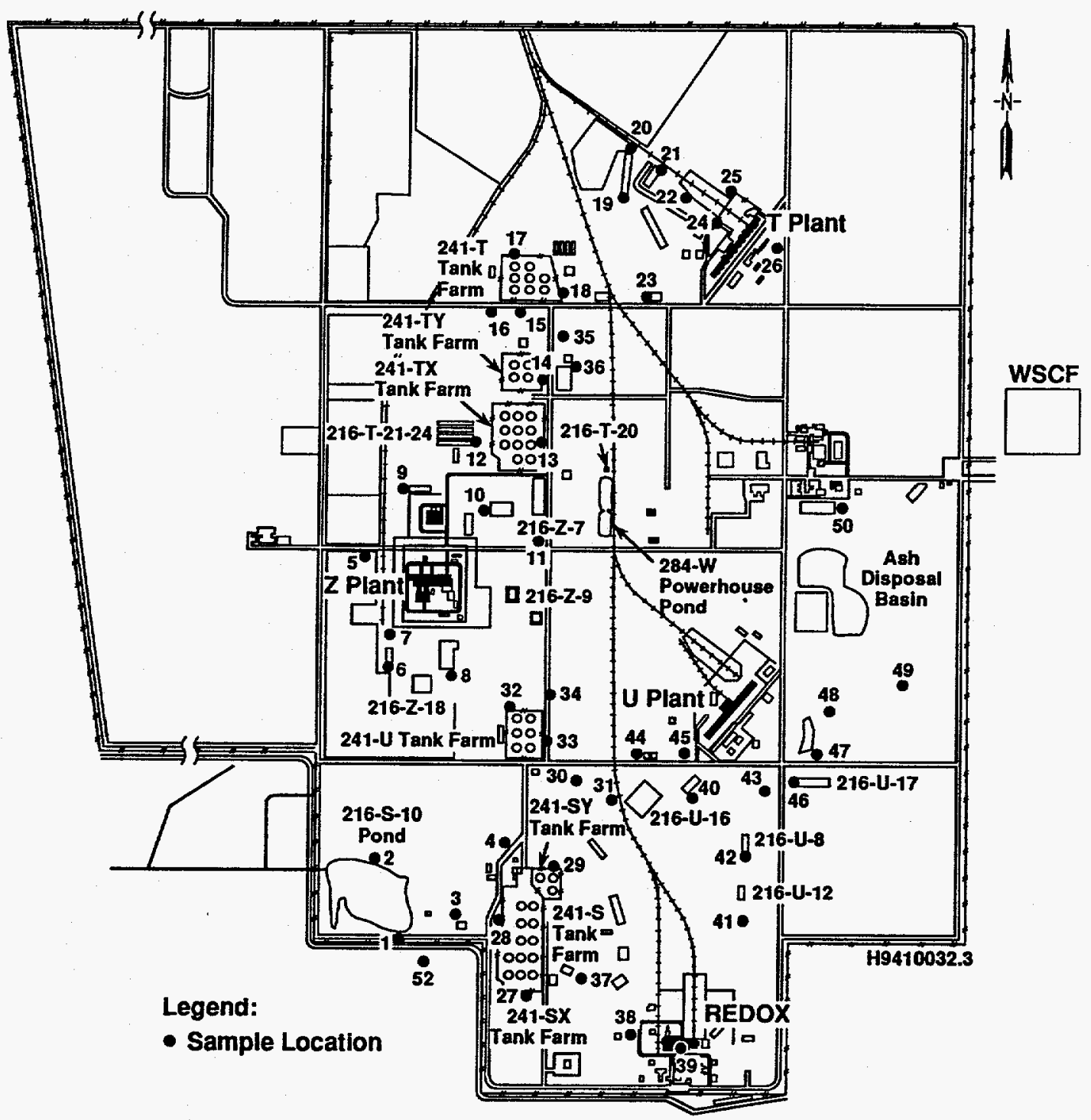


Figure A-49. 600 Area Soil and Vegetation Sample Locations.

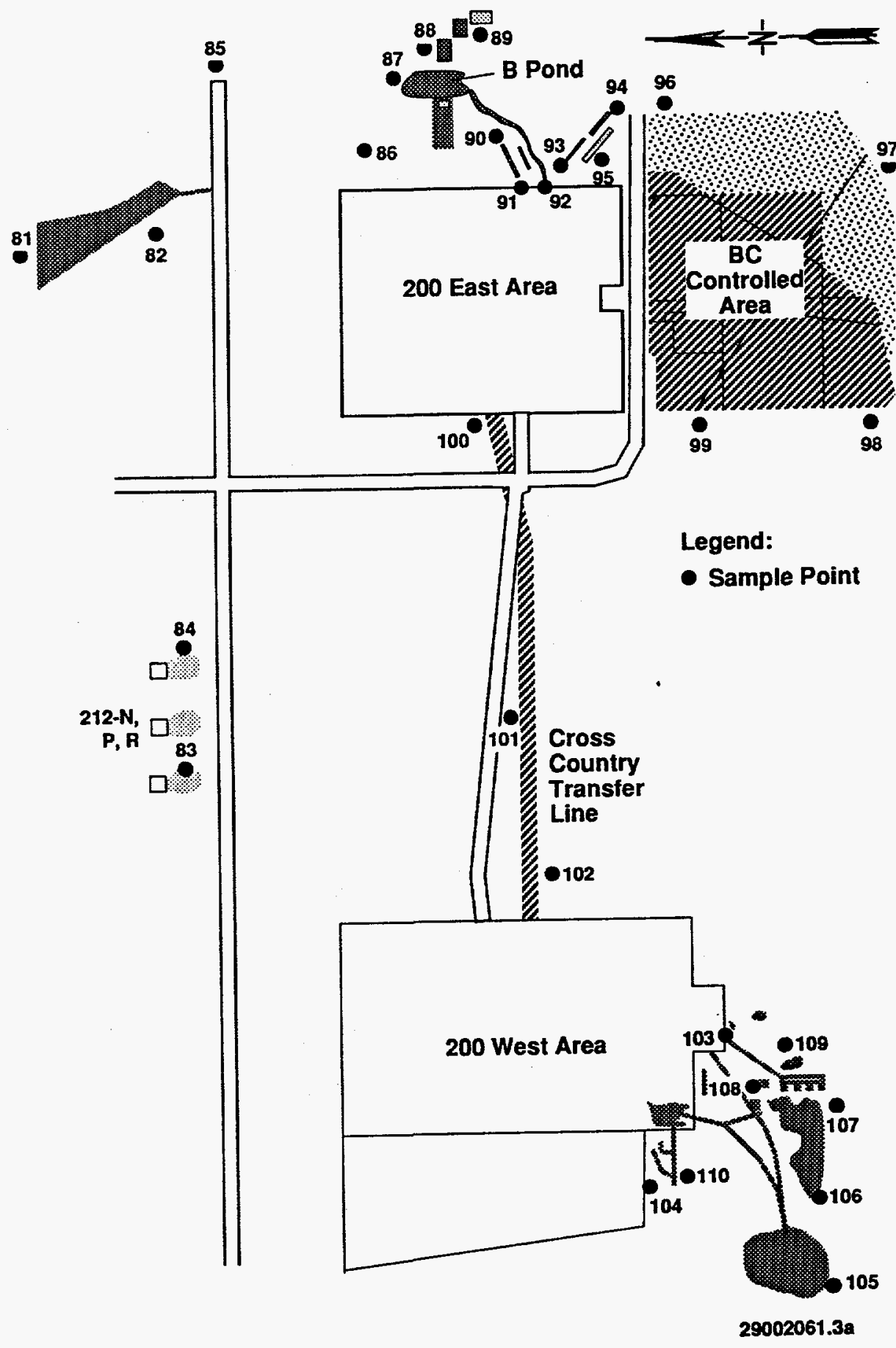


Figure A-50. 300 Area Soil and Vegetation Sample Locations.

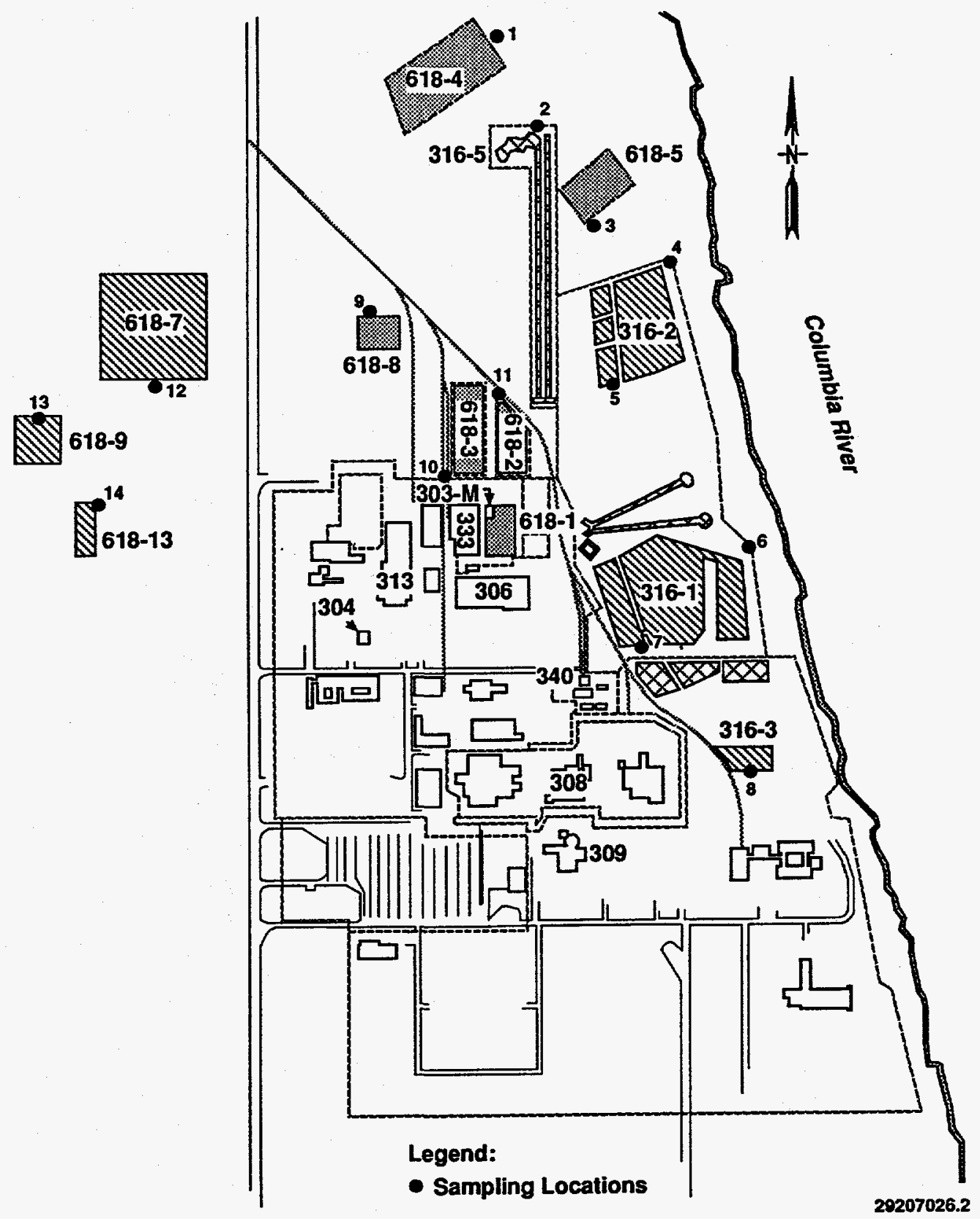


Figure A-51. 400 Area Soil and Vegetation Sample Locations.

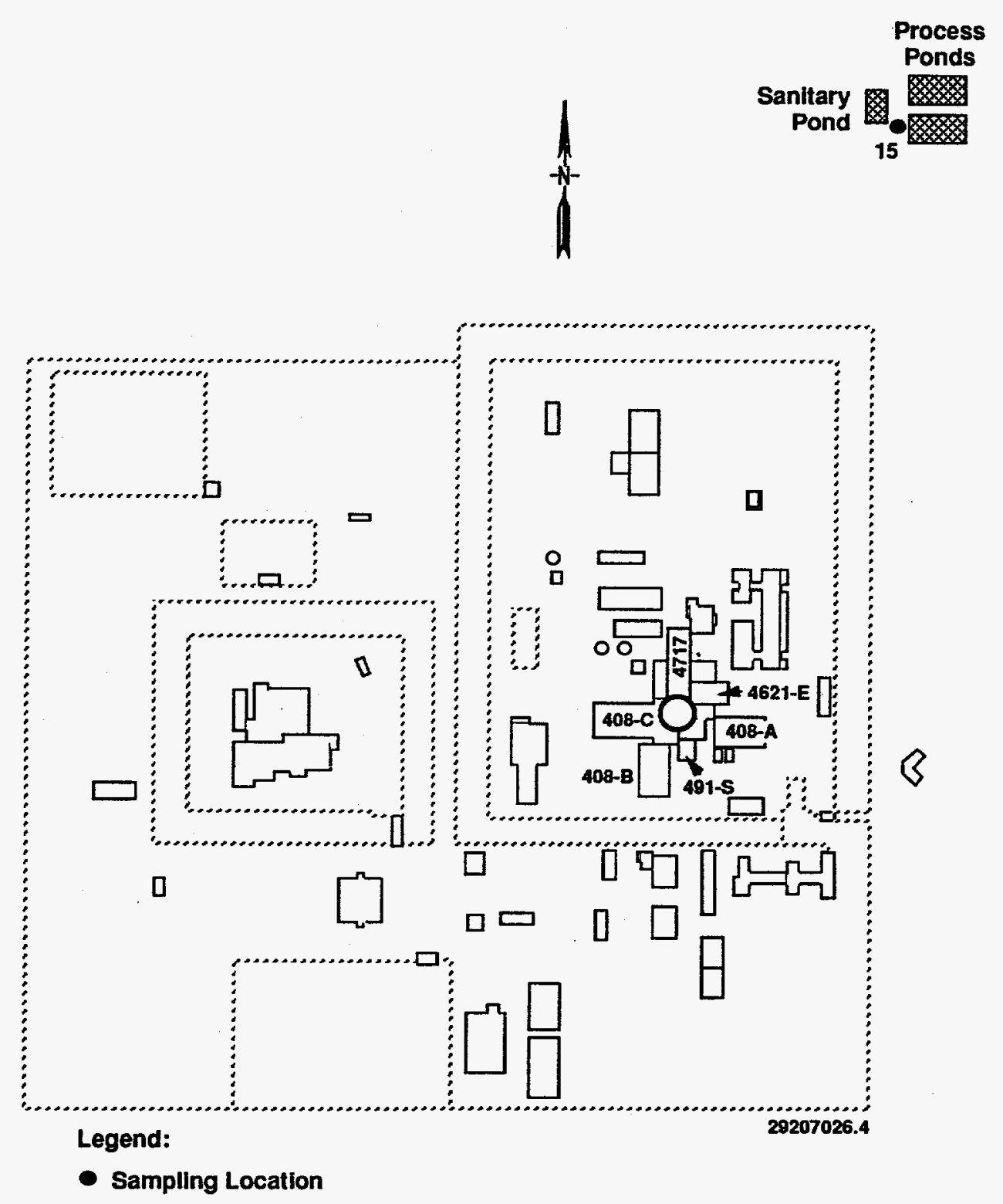


WHC-SP-0098-6

Figures A-52 through A-54 show water, sediment, and aquatic vegetation sample locations. 
Figure A-52. 200 East Water, Sediment, and Aquatic Vegetation Sample Locations.

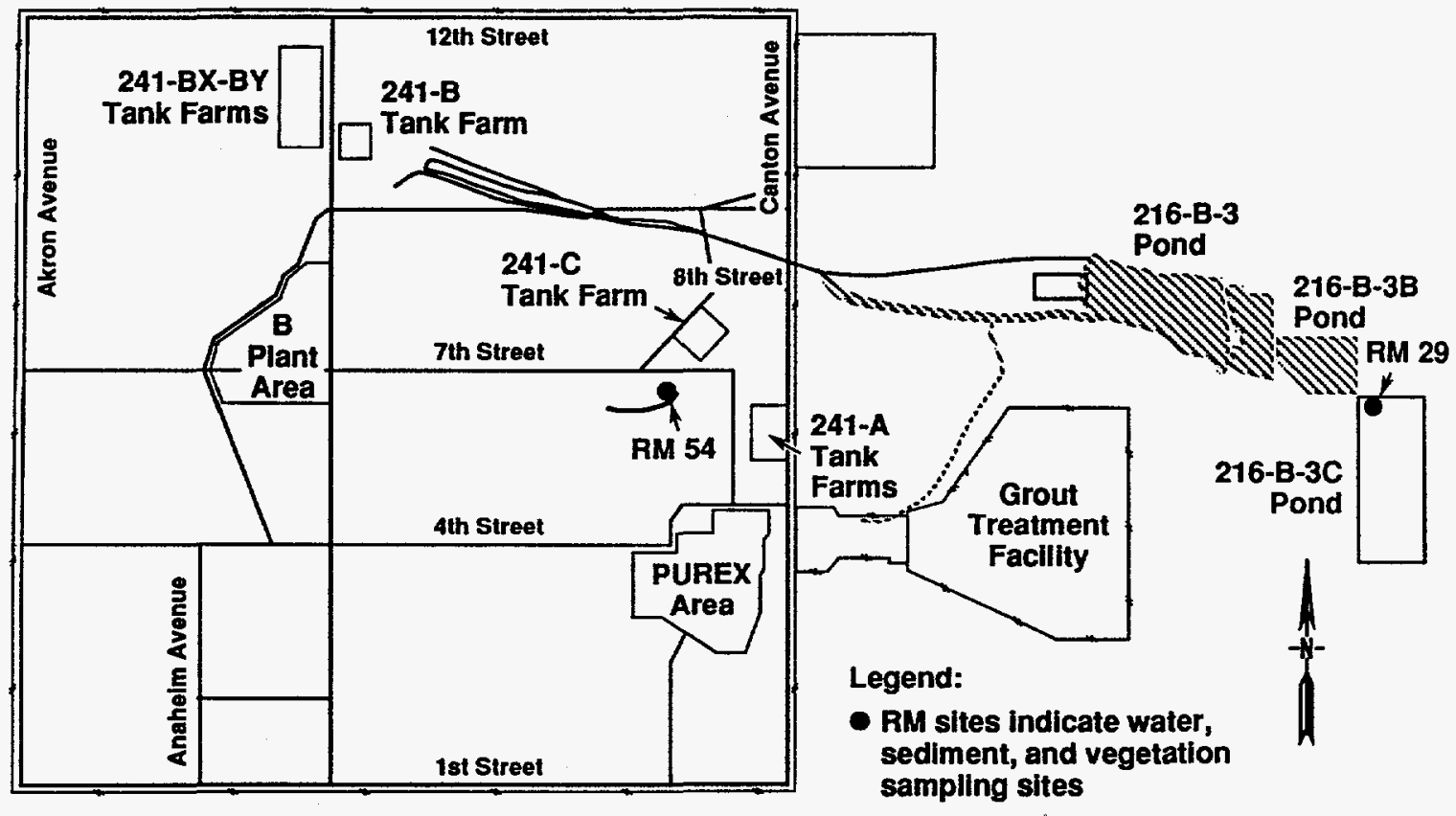

H9310036.7a 
Figure A-53. 200 West Water, Sediment, and Aquatic Vegetation Sample Locations.

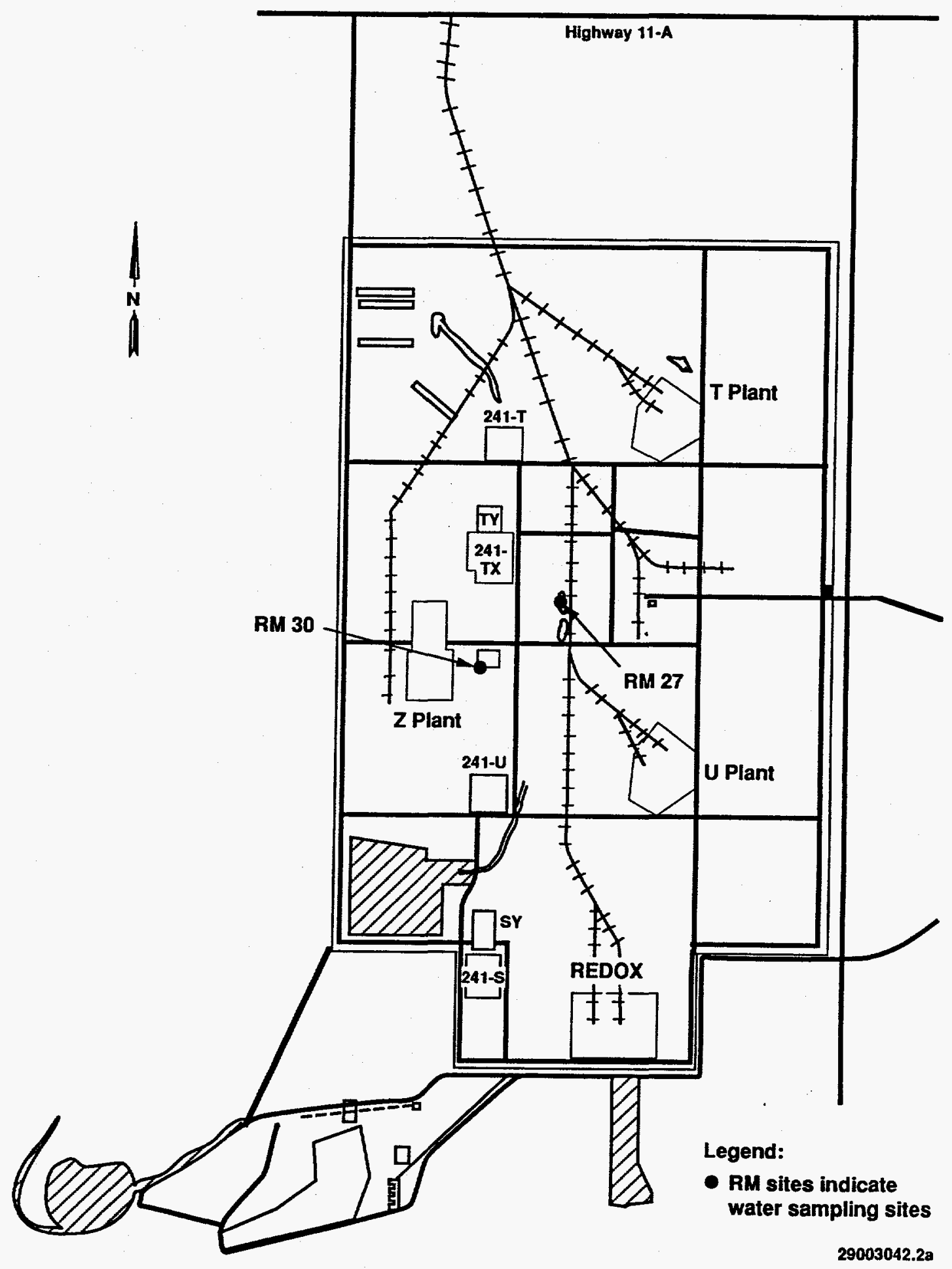


WHC-SP-0098-6

Figure A-54. N Springs Seepage Sample Locations.

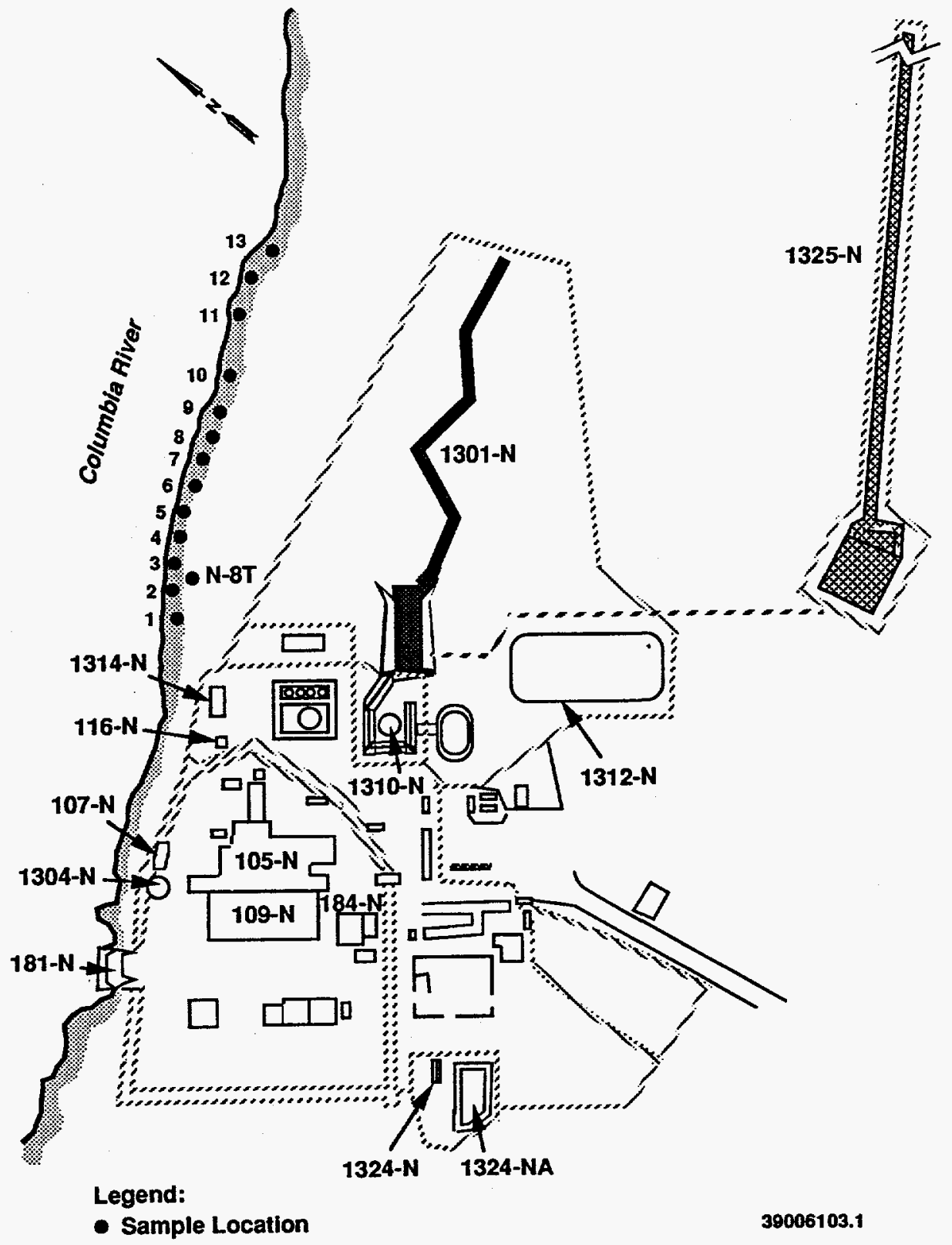


WHC-SP-0098-6

APPENDIX B

DESCRIPTIONS OF 200 AREA UNPLANNED RELEASE SITES 
WHC-SP-0098-6

This page intentionally left blank. 


\begin{tabular}{|c|c|c|}
\hline SITE & UPR NUMBER & SITE DESCRIPTION/LOCATION \\
\hline UN-216-E-1 & UPR-200-E-73 & $\begin{array}{l}\text { Area around the } 241-\mathrm{B}-151 \text { diversion box } \\
\text { within the } 241-\mathrm{B} \text { tank farm, contaminated with } \\
\text { approximate1y } 10 \text { C } \mathrm{C} \text { of mixed fission products } \\
\text { in } 1951 \text { and } 1952 \text {. These inactive units have } \\
\text { been isolated and weather covered. }\end{array}$ \\
\hline$U N-216-E-2$ & UPR-200-E-74 & $\begin{array}{l}50 \mathrm{ft}^{2} \text { of soil around the } 241-\mathrm{B}-152 \text { diversion } \\
\text { box, southwest corner of } 241-\mathrm{B} \text { tank farm, } \\
\text { contaminated with approximately } 1 \text { Ci of mixed } \\
\text { fission products in } 1954 \text {. These inactive } \\
\text { units have been isolated and weather covered. }\end{array}$ \\
\hline UN-216-E-3 & UPR-200-E-75 & $\begin{array}{l}50 \times 100 \mathrm{ft} \text { area of soil around the } 241-\mathrm{B}-153 \\
\text { diversion box, southwest corner of } 241-\mathrm{B} \text { tank } \\
\text { farm, contaminated with approximately } 1 \text { Ci of } \\
\text { mixed fission products in } 1954 \text { and } 1955 \text {. } \\
\text { These inactive units have been isolated and } \\
\text { weather covered. }\end{array}$ \\
\hline UN-216-E-4 & UPR-200-E-76 & $\begin{array}{l}\text { Area from the } 241-\mathrm{B}-153 \text { line break that } \\
\text { connected the } 9-2 \text { Tank in the } 221-\mathrm{B} \text { Plant to } \\
\text { the } 110-\mathrm{B} \text { Underground Storage Tank, } \\
\text { contaminated with approximate } 44,780 \mathrm{Ci} \\
{ }^{144} \mathrm{Ce}, 340 \mathrm{Ci}{ }^{106} \mathrm{Ru} \text {, and } 850 \mathrm{Ci} \mathrm{Zr} / \mathrm{Nb} \text { in } \\
1968 \text {. The contaminated soil was covered with } \\
\text { clean gravel. }\end{array}$ \\
\hline UN-216-E-5 & UPR-200-E-77 & $\begin{array}{l}\text { Area around the } 241-\mathrm{B}-154 \text { diversion box, } \\
\text { corner of Baltimore and } 7 \text { th Streets, } \\
\text { contaminated with approximately } 1 \text { Ci of mixed } \\
\text { fissile products in } 1946 \text {. This inactive unit } \\
\text { has been isolated and weather covered. }\end{array}$ \\
\hline UN-216-E-6 & UPR-200-E-78 & $\begin{array}{l}200 \mathrm{ft}^{2} \text { of soil around the } 241-\mathrm{B}-155 \\
\text { diversion box, } 900 \mathrm{ft} \text { south of } 241-\mathrm{BX} \text { tank } \\
\text { farm, contaminated with approximately } 10 \mathrm{Ci} \\
\text { of mixed fission products in 1955. This } \\
\text { inactive unit has been isolated and weather } \\
\text { covered. }\end{array}$ \\
\hline UN-216-E-7. & UPR-200-E-79 & $\begin{array}{l}\text { Area on the waste line that runs from } 242-B \\
\text { facility to } 207-\mathrm{B} \text { retention basin, } \\
\text { contaminated with approximately } 10 \mathrm{Ci} \text { of } \\
\text { mixed fission products in } 1953 \text {. Area was } \\
\text { covered with approximately } 2 \text { in. of clean } \\
\text { soil. No postings mark the area. }\end{array}$ \\
\hline
\end{tabular}




\begin{tabular}{|c|c|c|}
\hline SITE & UPR NUMBER & SITE DESCRIPTION/LOCATION \\
\hline UN-216-E-8 & UPR-200-E-80 & $\begin{array}{l}100 \times 500 \mathrm{ft} \text { area from } 221-\mathrm{B} \mathrm{R}-3 \text { line break, } \\
\text { south of } 221-\mathrm{B} \text { and north of } 7 \text { th Street, } \\
\text { contaminated with approximately } 10 \mathrm{Ci} \text { of } \\
\text { mixed fission products in } 1946 \text {. The ground } \\
\text { above the line break caved in, but was } \\
\text { backfilled with several feet of clean gravel. } \\
\text { The majority of the contaminated material was } \\
\text { moved to the } 200 \text { East Dry Waste Burial } \\
\text { Ground. Approximately } 5 \mathrm{Ci} \text { of material } \\
\text { remain. }\end{array}$ \\
\hline UN-216-E-9 & UPR-200-E-81 & 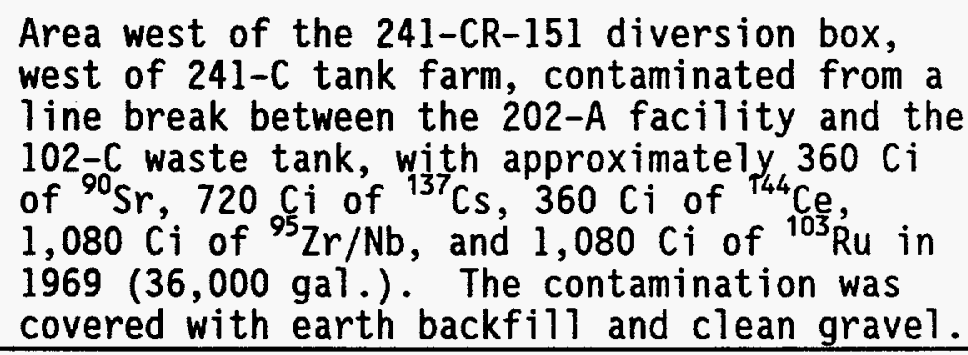 \\
\hline UN-216-E-10 & UPR-200-E-82 & $\begin{array}{l}5-\mathrm{ft}^{2} \text { area, northeast of the } 241-\mathrm{C} \text { tank farm } \\
\text { near the } 241-\mathrm{C}-152 \text { diversion box, } \\
\text { contaminated from a line leak between the } \\
241-\mathrm{C}-105 \text { tank to the } 221-\mathrm{B} \text { facility, with } \\
\text { approximately } 100 \mathrm{Ci} \text { of } 134 \mathrm{Cs}, 11300 \mathrm{Ci} \text { of } \\
{ }^{357} \mathrm{Cs}, 260 \mathrm{Cj} \text { of } 144 \mathrm{Ce}, 260 \mathrm{Ci} \text { of } 95 \mathrm{Zr} / \mathrm{Nb} \text {, and } \\
130 \mathrm{Ci} \text { of } 106 \mathrm{Ru}(2,600 \mathrm{gal} \text { ) in } 1969 \text {. Area } \\
\text { was cleaned during the decontamination outage } \\
\text { of } 241-\mathrm{C} \text { tank farm following the } 241-\mathrm{C}-15 \mathrm{l} \\
\text { release in } 1985 \text {. }\end{array}$ \\
\hline UN-216-E-11 & UPR-200-E-83 & $\begin{array}{l}4-\mathrm{mi}^{2} \text { area south of } 200 \text { East Area, } \\
\text { encompassing } \mathrm{BC} \text { cribs and trenches, } \\
\text { contaminated by radioactive rabbit and coyote } \\
\text { feces containing approximately } 14 \mathrm{Ci} \text { of } 135 \mathrm{Cs} \\
\text { and }{ }^{90} \text { Sr. Routine surveillance indicates the } \\
\text { contamination to be fixed beneath a good } \\
\text { growth of vegetation. No significant } \\
\text { evidence of resuspension. Area designated as } \\
\text { "BC-Controlled Area." }\end{array}$ \\
\hline UN-216-E-12 & UPR-200-E-84 & $\begin{array}{l}\text { Area approximately } 800 \mathrm{ft} \text { southwest of the } \\
221-\mathrm{B} \text { facility near the } 241-E R-151 \text { diversion } \\
\text { box had a release of approximately } 1,700 \mathrm{gal} \\
\text { from a leak in the catch tank in } 1953 \text {. No } \\
\text { contamination was detected. }\end{array}$ \\
\hline UN-216-E-13 & UPR-200-E-85 & $\begin{array}{l}\text { Area south of } 221-\mathrm{B} \text { facility, northwest } \\
\text { corner of the R-13 utility pit, contaminated } \\
\text { with approximately } 15 \mathrm{Ci} \text { of } 13 \mathrm{CS}^{\mathrm{C}} \mathrm{C} \text {, by a leak } \\
\text { in the pipeline from Tank } 18-1 \text { to the } 154-\mathrm{BX} \\
\text { diversion box in } 1972 \text {. Contamination levels } \\
\text { were reduced to approximately } 14 \mathrm{Ci} \text { by } \\
12 / 31 / 73 \text {. }\end{array}$ \\
\hline
\end{tabular}




\begin{tabular}{|c|c|c|}
\hline SITE & UPR NUMBER & SITE DESCRIPTION/LOCATION \\
\hline$U N-216-E-14$ & UPR-200-E-86 & $\begin{array}{l}\text { Area outside the southwest corner of the } \\
241-\mathrm{C} \text { tank farm, contaminated from a leak in } \\
\text { the vicinity of Line } \# 812 \text { ( } 8 \mathrm{ft} \text { deep) used to } \\
\text { transfer waste from AR vault to } 241-\mathrm{C} \text { tank } \\
\text { farm in } 1971 \text {. The line contained } 25,000 \mathrm{Ci} \\
\text { of }{ }^{137} \mathrm{Cs} \text {. }\end{array}$ \\
\hline UN-216-E-15 & UPR-200-E-87 & $\begin{array}{l}\text { Area on the south side of the } 224-\mathrm{B} \text { facility, } \\
\text { contaminated with approximately } 75 \mathrm{~g} \text { of }{ }^{239} \mathrm{Pu} \text {, } \\
\text { assumed to have resulted from leaks in the } \\
\text { underground pipe joints that entered the back } \\
\text { of } 224-\mathrm{B} \text { to feed its cells during processes } \\
\text { performed between } 1945 \text { and } 1953 \text {. }\end{array}$ \\
\hline UN-216-E-16 & UPR-200-E-88 & $\begin{array}{l}\text { Area about } 900 \mathrm{ft} \text { northwest of the 202-A } \\
\text { facility designated "TC-4 Railroad Spur," } \\
\text { contaminated by leakage from railroad } \\
\text { well/tank cars in } 1980 \text {. No contamination } \\
\text { levels recorded. }\end{array}$ \\
\hline UN-216-E-17 & UPR-200-E-89 & $\begin{array}{l}\text { Area bounding the north and northeast sides } \\
\text { of } 241-\mathrm{BY} \text { tank farm contaminated up to } 2,000 \\
\mathrm{cpm} \text { by airborne particulate matter, } \\
\text { resuspended by wind from activities during } \\
\text { the time of } 241-\mathrm{BY} \text { operations (reported in } \\
\text { 1978). Interim stabilization occurred in } \\
1991 \text {. }\end{array}$ \\
\hline UN-216-E-18 & UPR-200-E-90 & $\begin{array}{l}\text { Area adjacent to the } 291-B \text { stack sand filter, } \\
\text { about } 300 \text { ft south of the } 221-B \text { Facility. } \\
\text { Contamination possibly spread from Unplanned } \\
\text { Release Site UN-216-E-8 (UPR-200-E-80) in } \\
1980 \text {. No contamination levels recorded. }\end{array}$ \\
\hline UN-216-E-19 & UPR-200-E-91 & $\begin{array}{l}\text { Area adjacent to the northeast corner of the } \\
241-C \text { tank farm, contaminate by downhill } \\
\text { seepage of the decontamination station, vapor } \\
\text { emissions, and windblown particulate matter } \\
\text { from inside the tank farm in } 1980 \text {. } \\
\text { Contaminated soil was excavated and buried in } \\
\text { the location now designated UN-216-E-33 (no } \\
\text { "UPR" number assigned) adjacent to the north } \\
\text { side of } 216-A-24 \text { crib. }\end{array}$ \\
\hline$U N-216-E-20$ & UPR-200-E-92 & $\begin{array}{l}\text { Area along the eastern outer perimeter of } 200 \\
\text { East Area, contaminated by windblown Russian } \\
\text { thistle from various waste sites in the area. } \\
\text { The thistle decomposed on the fencel ine and } \\
\text { infiltrated the soil in } 1980 \text {. Contaminated } \\
\text { soil was excavated and buried in the location } \\
\text { now designated UN-216-E-33 (no "UPR" number } \\
\text { assigned) adjacent to the north side of } \\
216-A-24 \text { crib. Area has been removed from } \\
\text { posting. }\end{array}$ \\
\hline
\end{tabular}


WHC-SP-0098-6

\begin{tabular}{|c|c|c|}
\hline SITE & UPR NUMBER & SITE DESCRIPTION/LOCATION \\
\hline UN-216-E-21 & UPR-200-E-93 & $\begin{array}{l}\text { Area along the eastern inner perimeter of } 200 \\
\text { East, contaminated by windblown Russian } \\
\text { thistle from various waste sites in the area. } \\
\text { The thistle decomposed on the fencel ine and } \\
\text { infiltrated the soil in } 1980 \text {. Contaminated } \\
\text { soil was excavated and buried in the location } \\
\text { now designated UN-216-E-33 (no "UPR" number } \\
\text { assigned) adjacent to the north side of } \\
216-A-24 \text { crib. Area has been removed from } \\
\text { posting. }\end{array}$ \\
\hline UN-216-E-22 & UPR-200-E-94 & $\begin{array}{l}\text { Gravel pit east of } 200 \text { East Area and north of } \\
216-\mathrm{B}-3-1 \text { ditch diverter station, } \\
\text { contaminated when decontamination efforts of } \\
\text { earthmoving equipment (contaminated up to } \\
8,000 \mathrm{cpm} \text { ) left a } 300 \mathrm{cpm} \text { residue at the } \\
\text { bottom of the pit in } 1979 \text {. Area has been } \\
\text { removed from posting. }\end{array}$ \\
\hline UN-216-E-23 & UPR-200-E-95 & $\begin{array}{l}\text { Railroad spur north of } 221-B \text { facility and } \\
\text { between } 218-E-2 A \text { and } 218-E-5 \text { burial grounds, } \\
\text { assumed contaminated in excess of } 100,000 \mathrm{cpm} \\
\text { by leaking railroad tank/well cars. } \\
\text { Established as an Unplanned Release Site in } \\
1980 \text {. }\end{array}$ \\
\hline UN-216-E-24 & UPR-200-E-96 & $\begin{array}{l}\text { Area southeast of the 202-A facility (PUREX) } \\
\text { to the protected area southern fenceline, } \\
\text { contaminated from the residue produced by } \\
291-A \text { stack and diversion box work during the } \\
\text { operational years of the facility (reported } \\
\text { in 1980). No contamination levels reported. }\end{array}$ \\
\hline UN-216-E-25 & UPR-200-E-97 & $\begin{array}{l}\text { Area of unknown origin south of the 202-A } \\
\text { facility near the railroad tunnel. The } \\
\text { surface contamination was removed during the } \\
\text { construction of the double-exclusion fence } \\
\text { and posting was removed when area was } \\
\text { established as an Unplanned Release Site in } \\
1980 \text {. No contamination levels reported. }\end{array}$ \\
\hline UN-216-E-26 & UPR-200-E-98 & $\begin{array}{l}\text { Area east of the } C \text { facility (hot semiworks) } \\
\text { near the base of the } 291-C \text { stack and around } \\
\text { the } 216-C-2 \text { reverse wel1, contaminated from } \\
\text { particulate matter spread from operation of } \\
\text { the facility between } 1955 \text { and } 1965 \text {. No } \\
\text { contamination levels reported. The } \\
\text { contamination has been removed and the site } \\
\text { stabilized. }\end{array}$ \\
\hline
\end{tabular}




\begin{tabular}{|c|c|c|}
\hline SITE & UPR NUMBER & SITE DESCRIPTION/LOCATION \\
\hline UN-216-E-27 & UPR-200-E-99 & $\begin{array}{l}\text { Portion of the ground surface surrounding the } \\
244-C R \text { vault, in the south corner of } 241-C \\
\text { tank farm, contaminated during numerous } \\
\text { piping changes associated with the facility. } \\
\text { No contamination levels reported. The site } \\
\text { was decontaminated in 1981. }\end{array}$ \\
\hline UN-216-E-28 & $N / A$ & This number not assigned. \\
\hline UN-216-E-29 & UPR-200-E-100 & $\begin{array}{l}\text { Area south and east of } 241-C \text { tank farm } \\
\text { (corner of } 7 \text { th Street and Buffalo), assumed } \\
\text { contaminated by windblown particulates and } \\
\text { biological transport from the tank farm. The } \\
\text { area has merged with UN-216-E-43, which } \\
\text { surrounds most of the } 244-A \text { ift station and } \\
216-A-40 \text { retention basin. Decontamination } \\
\text { and decommissioning efforts were active as of } \\
4 / 1 / 94 \text {. }\end{array}$ \\
\hline$U N-216-E-30$ & UPR-200-E-101 & $\begin{array}{l}\text { Area between } 242-B \text { evaporator and the } 241-B \\
\text { tank farm fence, possibly contaminated by } \\
\text { windblown particulates from the tank farm or } \\
\text { spills out of the } 242-b \text { Evaporator. The } \\
\text { contamination has been removed and the site } \\
\text { stabilized. }\end{array}$ \\
\hline UN-216-E-31 & UPR-600-E-21 & $\begin{array}{l}\text { Area northeast of } 200 \text { East Area, size and } \\
\text { boundaries not determined, contaminated in } \\
\text { excess of } 100,000 \mathrm{cpm} \text { with windblown Russian } \\
\text { thistle and decomposed Russian thistle from } \\
\text { various facilities within } 200 \text { East Area. The } \\
\text { contamination was removed from the most } \\
\text { concentrated areas in } 1986 \text {, but the area is } \\
\text { too large for unrestricted release. }\end{array}$ \\
\hline UN-216-E-32 & $N / A$ & $\begin{array}{l}\text { Area adjacent to and northeast of the } \\
218-E-12 B \text { burial ground. This area has been } \\
\text { incorporated into the expansion of the } \\
218-E-12 B \text { burial ground. An accurate history } \\
\text { of the area is unknown. }\end{array}$ \\
\hline UN-216-E-33 & $N / A$ & $\begin{array}{l}\text { Area adjacent and north of the } 216-\mathrm{A}-24 \mathrm{crib} \text {, } \\
\text { contaminated by biological transport of } \\
\text { contamination buried there during } \\
\text { decontamination of other problem areas. The } \\
\text { contamination was buried under approximately } \\
\text { four feet of clean fill in } 1988 \text { and posted as } \\
\text { URM. }\end{array}$ \\
\hline UN-216-E-34 & $N / A$ & $\begin{array}{l}\text { Area southeast of 202-A (PUREX) facility, } \\
\text { outside the exclusion area, contamination } \\
\text { possibly caused by } 291-A \text { stack emissions. No } \\
\text { contamination levels reported. }\end{array}$ \\
\hline
\end{tabular}


WHC-SP-0098-6

\begin{tabular}{|c|c|c|}
\hline SITE & UPR NUMBER & SITE DESCRIPTION/LOCATION \\
\hline UN-216-E-35 & $N / A$ & $\begin{array}{l}\text { Area northwest of } 216-B-3-1 \text { and } 216-B-3-2 \\
\text { backfield ditches. }\end{array}$ \\
\hline UN-216-E-36 & $N / A$ & West side of the 216-B-64 basin. \\
\hline UN-216-E-37 & $N / A$ & $\begin{array}{l}\text { Area east of hot-semiworks. Area cleaned up } \\
\text { and release from all posting in } 1991 \text {. }\end{array}$ \\
\hline UN-216-E-38 & $N / A$ & Area around hot-semiworks building. \\
\hline UN-216-E-39 & $N / A$ & $\begin{array}{l}\text { Area east of hot-semiworks, south of } 7 \text { th Ave } \\
\text { and north of the powerhouse ditch. Area was } \\
\text { decontaminated and released from posting in } \\
1989 \text {. Contamination was placed in } 218-C-9 \\
\text { burial ground before it was stabilized. }\end{array}$ \\
\hline$U N-216-E-40$ & $N / A$ & $\begin{array}{l}\text { Area north of the } 216-B-3-3 \text { ditch east of } 200 \\
\text { East Area. }\end{array}$ \\
\hline UN-216-E-41 & UPR-600-20 & $\begin{array}{l}\text { The cross site transfer line, including the } \\
241-\text {-ER-151 vent station, contaminated by } \\
\text { biological transport of radioactive materials } \\
\text { that leaked into the pipeline encasement and } \\
\text { windblown particulates from the vent station. } \\
\text { No contamination levels reported. }\end{array}$ \\
\hline UN-216-E-42 & $N / A$ & $\begin{array}{l}\text { Number assigned then canceled. This site was } \\
\text { the same as UN-216-E-36. }\end{array}$ \\
\hline UN-216-E-43 & UPR-200-E-143 & $\begin{array}{l}\text { Area } 50 \mathrm{ft} \text { south of } 244-\mathrm{A} \text { lift station and } \\
100 \mathrm{ft} \text { east of } 216-\mathrm{A}-40 \text { basin. }\end{array}$ \\
\hline UN-216-E-44 & UPR-200-E-144 & $\begin{array}{l}\text { Area north and east of } 241-B \text { tank farm. This } \\
\text { area of migration covers about } 12 \text { acres. } \\
\text { Area was interim stabilized and released from } \\
\text { posting status in } 1992 \text {. }\end{array}$ \\
\hline UN-216-W-2 & UPR-200-W-95 & $\begin{array}{l}\text { Area inside the } 207-5 \text { retention basin, } \\
\text { contaminated with approximately } 10 \mathrm{Ci} \text { of } \\
\text { mixed fission products in } 1954 \text { when coil } \\
\text { leaks in the } 202-5 \text { Facility contaminated } \\
\text { cooling water. The basin was buried with } \\
\text { clean soil in 1993. }\end{array}$ \\
\hline$U N-216-W-4$ & UPR-200-W-96 & $\begin{array}{l}\text { Area adjacent to and directly north of the } \\
233-5 \text { filter house, contaminated with } \\
\text { approximately } 0.1 \mathrm{~g} \text { of } 239 \mathrm{Pu}(>40,000 \mathrm{dpm}) \\
\text { when contaminated water backed up in the } \\
\text { filter house drain and overflowed into a low } \\
\text { spot in the ground in } 1969 \text {. The area was } \\
\text { originally covered with } 18 \text { in. of washed } \\
\text { gravel and was ultimately covered with an } \\
\text { asphalt roadway. }\end{array}$ \\
\hline
\end{tabular}




\begin{tabular}{|c|c|c|}
\hline SITE & UPR NUMBER & SITE DESCRIPTION/LOCATION \\
\hline$U N-216-W-5$ & UPR-200-W-97 & $\begin{array}{l}\text { Area on the southeast corner of } 23 \mathrm{rd} \text { St and } \\
\text { Camden Ave, contaminated with approximately } \\
10 \mathrm{Ci} \text { of fission products in } 1954 \text { when an } \\
\text { underground pipeline between } 152-T \text { and } 153-\mathrm{TX} \\
\text { broke and again when it was mistakenly reused } \\
\text { in } 1966 \text {. The area was excavated to } 3 \text { to } 4 \mathrm{ft} \\
\text { and covered with clean soil. }\end{array}$ \\
\hline$U N-216-W-6$ & UPR-200-W-98 & $\begin{array}{l}\text { Area at the southeast corner of the } 221-T \\
\text { facility, near section R19, contaminated with } \\
\text { mixed fission products, which surfaced from a } \\
\text { broken process waste transfer line in } 1945 \text {. } \\
\text { Russian thistle contaminated with }{ }^{90} \text { Sr and } \\
{ }^{137} \text { Cs was discovered growing on the spill site } \\
\text { in } 1968 \text {, but ensuing crops became less } \\
\text { contaminated until no contamination was found } \\
\text { in } 1975 \text {. The area was originally covered } \\
\text { with } 4 \text { ft of clean soil, and has since been } \\
\text { covered by an asphalt roadway. }\end{array}$ \\
\hline UN-216-W-7 & UPR-200-W-99 & $\begin{array}{l}\text { Area } 750 \mathrm{ft} \text { north and south along Camden Ave, } \\
\text { extending } 75 \text { to } 100 \mathrm{ft} \text { east of the roadway, } \\
\text { contaminated with approximately } 1 \mathrm{Ci} \text { of } 90 \mathrm{Sr} \\
\text { (up to } 700 \mathrm{mrad} / \mathrm{hr} \text { ) when two plumes of } \\
\text { airborne particulates escaped from } 153-\mathrm{TX} \\
\text { diversion box in } 1966 \text {. The area on the west } \\
\text { side of Camden was covered with gravel, but } \\
\text { was recontaminated by windblown particulates } \\
\text { from } 241-T X \text { tank farm in } 1993 \text {. The area on } \\
\text { the east side of Camden was covered with } \\
\text { approximately } 4 \mathrm{ft} \text { of clean soil and } \\
\text { revegetated in } 1990 \text {. }\end{array}$ \\
\hline$U N-216-W-8$ & UPR-200-W-100 & $\begin{array}{l}\text { Area in } 241-T X \text { tank farm, on the process line } \\
\text { between } 241-T X-105 \text { and } 241-T X-118 \text { tanks, } \\
\text { contaminated with approximately } 10 \mathrm{Ci} \text { of } \\
\text { fission products with a maximum dose rate of } \\
4.5 \mathrm{R} / \mathrm{hr} \text { at } 4 \mathrm{ft} \text { in } 1954 \text {. The contaminated } \\
\text { area was covered with } 1 \mathrm{ft} \text { of clean soil. }\end{array}$ \\
\hline$U N-200-W-8$ & UPR-200-W-8 & $\begin{array}{l}\text { Area near the northeast corner of Beloit Ave } \\
\text { and 15th St, designated "The 01d Burning } \\
\text { Ground," contaminated with approximately } 1 \mathrm{Ci} \\
\text { of fission products with a maximum dose rate } \\
\text { of } 45 \mathrm{R} / \mathrm{hr} \text { at the surface, from an unknown } \\
\text { source in } 1950 \text {. The area has been covered } \\
\text { with } 10 \mathrm{ft} \text { of clean soil. }\end{array}$ \\
\hline
\end{tabular}


WHC-SP-0098-6

\begin{tabular}{|c|c|c|}
\hline SITE & UPR NUMBER & SITE DESCRIPTION/LOCATION \\
\hline$U N-216-W-9$ & UPR-200-W-101 & $\begin{array}{l}\text { Area on the southeast side of } 221-U \text { facility, } \\
\text { between sections Rl-R5, contaminated with } \\
\text { recllaimed acid containing approximately } 1 \mathrm{Ci} \\
\text { of } 90 \mathrm{Sr} \text { that was spilled on the ground in } \\
1957 \text {. Radiological surveys performed in } 1976 \\
\text { revealed } 300 \mathrm{cpm} \text { on the surface. The area } \\
\text { was covered with } 3 \mathrm{in} \text {. of sand and gravel. }\end{array}$ \\
\hline$U N-216-W-10$ & NA & $\begin{array}{l}\text { Area approximately } 500 \mathrm{ft} \text { west of } 271-\mathrm{U} \\
\text { facility and } 550 \mathrm{ft} \text { north of } 16 \mathrm{th} \mathrm{St} \text {. Cannot } \\
\text { locate. No additional information. }\end{array}$ \\
\hline UN-216-W-11 & UPR-200-W-138 & $\begin{array}{l}\text { Area at the northwest corner of } 221-U \\
\text { facility near section R3, contaminated when } \\
\text { an estimated } 300 \text { 1b of uranium (UNH solution) } \\
\text { overflowed into the } 221-U \text { building vessel } \\
\text { vent blower pit and then went to ground } \\
\text { through the French drain in } 1953 \text {. No record } \\
\text { of decontamination efforts. }\end{array}$ \\
\hline UN-216-W-12 & UPR-200-W-102 & $\begin{array}{l}50-X 12-X 12-\mathrm{ft} \text { area on the southeast side } \\
\text { of the } 224-\mathrm{f} \text { facility, contaminated with } \\
\text { about } 10 \mathrm{~g} \text { of }{ }^{239} \mathrm{Pu} \text {, when alpha-laden moisture } \\
\text { seeped through the jointed tile piping from } \\
\text { process tanks entering the rear of the } \\
\text { building at ground level during the years of } \\
\text { process operations (discovered in 1972). } 139 \\
\text { drums of soil were excavated and removed for } \\
\text { burial. }\end{array}$ \\
\hline UN-216-W-13 & UPR-200-W-103 & $\begin{array}{l}\text { Area about } 6 \mathrm{ft} \text { south and } 12 \mathrm{ft} \text { west of the } \\
236-7 \mathrm{facility} \text {, contaminated with about } 10 \mathrm{~g} \\
\text { of }{ }^{239} \mathrm{Pu}\left(7.6 \mathrm{E}+07 \mathrm{dpm} / 100 \mathrm{~cm}^{3} \text { of ground) when }\right. \\
\text { the } 216-\mathrm{Z}-18 \mathrm{crib} 1 \mathrm{ine} \text { from } 234-5 \mathrm{facility} \\
\text { broke in } 1971 \text {. An area } 25 \mathrm{ft} \text { long by } 6 \mathrm{ft} \\
\text { wide by } 7 \mathrm{ft} \text { deep was excavated, producing } \\
\text { approximately } 10055-\mathrm{gal} \text { drums of soil, which } \\
\text { were buried in the } 200 \text { West area plutonium } \\
\text { "storage for recovery" burial ground. Much } \\
\text { contamination remains under } 6 \mathrm{ft} \text { of clean } \\
\text { soil. }\end{array}$ \\
\hline$U N-216-W-14$ & UPR-200-W-104 & $\begin{array}{l}580-\mathrm{ft}-\text { long by } 40-\mathrm{ft} \text {-wide by } 10-\mathrm{ft} \text {-deep } \\
\text { trench running northwest from the northeast } \\
\text { corner of } 216-\mathrm{U}-10 \text { pond, dug to give } \\
\text { additional 1eaching surface for overflow } \\
\text { water, contaminated to about } 2,000 \mathrm{cpm} \text { in the } \\
\text { bottom of the trench. Maximum soil sample } \\
\text { analyses were } 5.2 \mathrm{E}+00 \mathrm{pCi} / \mathrm{g}^{90} \mathrm{Sr}, 1.9 \mathrm{E}+03 \\
\mathrm{pCi} / \mathrm{g}{ }^{137} \mathrm{Cs}, 2.8 \mathrm{E}+04 \mathrm{pCi} / \mathrm{g}{ }^{41} \mathrm{Am} \text { in } 1978 \text {. } \\
\text { Area was buried under about } 4 \mathrm{ft} \text { of clean } \\
\text { soil. } * \star\end{array}$ \\
\hline
\end{tabular}




\begin{tabular}{|c|c|c|}
\hline SITE & UPR NUMBER & SITE DESCRIPTION/LOCATION \\
\hline UN-216-W-15 & UPR-200-W-105 & $\begin{array}{l}500-\mathrm{ft}-1 \text { ong by } 45-\mathrm{ft} \text {-wide by } 15-\mathrm{ft} \text {-deep } \\
\text { trench running east from the center of the } \\
\text { east side of } 216-\mathrm{U}-10 \text { pond, dug to give } \\
\text { additional leaching surface for overflow } \\
\text { water, contaminated to about } 3,000 \mathrm{cpm} \text { in the } \\
\text { bottom of the trench. Maximum soil sample } \\
\text { analyses were } 8 \mathrm{O} \mathrm{E}+01 \mathrm{pCi} / \mathrm{g}{ }^{90} \mathrm{Sr} \text { and } \\
2.0 \mathrm{E}+03 \mathrm{pCi} / \mathrm{g}{ }^{37} \mathrm{Cs} \text { in } 1978 \text {. Area was buried } \\
\text { under about } 4 \mathrm{ft} \text { of clean soil.** }\end{array}$ \\
\hline UN-216-W-16 & UPR-200-W-106 & $\begin{array}{l}400-\mathrm{ft}-1 \text { ong by } 25-\mathrm{ft} \text {-wide by } 8-\mathrm{ft} \text {-deep trench } \\
\text { running east from the southeast side of } \\
216-U-10 \text { pond, dug to give additional } \\
\text { leaching surface for overflow water, } \\
\text { contaminated to about } 2,000 \mathrm{cpm} \text { in the bottom } \\
\text { of the trench. Maximum soil analyses were } \\
5.9 \mathrm{E}+01 \mathrm{pCi} / \mathrm{g}{ }^{90} \mathrm{Sr} \text { and } 1.4 \mathrm{E}+03 \mathrm{pCi} / \mathrm{g}{ }^{137} \mathrm{Cs} \\
\text { in } 1978 \text {. Area was buried under about } 4 \mathrm{ft} \text { of } \\
\text { clean soil. }\end{array}$ \\
\hline$U N-216-W-17$ & UPR-200-W-107 & $\begin{array}{l}\text { Area south of } 216-U-10 \text { pond, inundated by } \\
\text { rising water from the pond, which received } \\
\text { waste water from the } 216-U-14 \text { laundry ditch, } \\
\text { the } 216-Z-11 \text { ditch, and cooling water from } \\
\text { the } 401-S X \text { building condensers in the } 241-S x \\
\text { tank farm. Contamination reached a maximum } \\
\text { leve } 1 \text { of } 8,000 \mathrm{cpm} \text { on the surface of the } \\
\text { ground } \text { Maximum soil analyses were } 9.8 \mathrm{E}+01 \\
\text { pCi/g }{ }^{9} \mathrm{Sr} \text { and } 2.6 \mathrm{E}+03 \mathrm{pC} / \mathrm{g}{ }^{137} \mathrm{Cs} \text { in } 1978 \text {. } \\
\text { Area was buried under about } 4 \mathrm{ft} \text { of clean } \\
\text { soil. }\end{array}$ \\
\hline$U N-216-W-18$ & UPR-200-W-108 & $\begin{array}{l}\text { Area at the south end of } 216-S-9 \mathrm{crib} \\
\text { contaminated with an unknown amount of } \\
\text { radioactive material ( } 40 \mathrm{R} / \mathrm{hr} \text { from the bottom } \\
\text { of the waste line) when REDOX (202-S) process } \\
\text { condensate from the D-2 receiver tank flowed } \\
\text { from the } 240-S \text { Diversion Box to the "Y" in } \\
\text { the line between the } 216-S-9 \text { crib and the } \\
216-S-23 \text { crib, which was found to be buckled } \\
\text { in two places in } 1969 \text {. The waste water was } \\
\text { allowed to seep into the bottom of the } 20-\mathrm{ft}- \\
\text { deep hole, which was dug to maintain the } \\
\text { line, and filled after the work was complete. } \\
\text { Area is still a Surface Contamination Area } \\
\text { within the } 216-S-9 \text { crib boundary. }\end{array}$ \\
\hline
\end{tabular}


WHC-SP-0098-6

\begin{tabular}{|c|c|c|}
\hline SITE & UPR NUMBER & SITE DESCRIPTION/LOCATION \\
\hline UN-216-W-19 & UPR-200-W-109 & $\begin{array}{l}\text { Area inside the east perimeter of } 218-W-9 \\
\text { burial ground, contaminated with an unknown } \\
\text { amount of radioactive material ( } 40 \text { R/hr from } \\
\text { the bottom of the waste } 1 \text { ine) when REDOX } \\
\text { (202-S) process condensate from the D-2 } \\
\text { receiver tank flowed from the } 240-S \text { diversion } \\
\text { box to the "Y" in the line between the } \\
216-S-9 \text { crib and the } 216-S-23 \text { crib, which was } \\
\text { found to be buckled in two places in } 1969 \text {. } \\
\text { The waste water was allowed to seep into the } \\
\text { bottom of the } 20-f t-d e e p \text { hole, which was dug } \\
\text { to maintain the } 1 \text { ine, and filled after the } \\
\text { work was complete. The area was covered with } \\
\text { clean soil and revegetated along with } \\
218-W-9 \text {. }\end{array}$ \\
\hline UN-216-W-20 & UPR-200-W-110 & $\begin{array}{l}\text { A covered area (216-Z-1 ditch), } 425 \mathrm{ft} \text { long } \\
\text { by } 15 \mathrm{ft} \text { deep adjacent and parallel to the } \\
\text { head-end (north) of the } 216-Z-19 \text { ditch, } \\
\text { contaminated up to } 100,000 \mathrm{dpm} \text { (alpha) by } \\
\text { intrusion of process leaks into the } 234-5 Z \\
\text { Plant cooling water discharge system, which } \\
\text { settled out or was absorbed by aquatic } \\
\text { vegetation along the bottom and sides of } \\
216-Z-1 \text { ditch, mistakenly uncovered during } \\
\text { the construction of the new } 216-Z-19 \text { ditch in } \\
1971 \text {. The soil was assumed to have been } \\
\text { returned to its original location when it was } \\
\text { decided to construct the } 216-Z-19 \text { ditch } 35 \mathrm{ft} \\
\text { further west. }\end{array}$ \\
\hline$U N-216-W-21$ & UPR-200-W-111 & $\begin{array}{l}40-\mathrm{ft}-1 \text { ong by } 8-10-\mathrm{ft}-\mathrm{deep} \text { by } 15-\mathrm{ft} \text {-wide area } \\
\text { within } 10 \mathrm{ft} \text { of the south wall of the } 207-U \\
\text { retention basin, constructed to contain } \\
\text { sludge from the bottom of the southern } \\
\text { section of } 207-U \text { retention basin at an } \\
\text { unknown time. The sludge was originally } \\
\text { covered with } 4 \mathrm{ft} \text { of clean soil, but has been } \\
\text { recontaminated and is presently a Surface } \\
\text { Contamination Area. }\end{array}$ \\
\hline$U N-216-W-22$ & UPR-200-W-112 & $\begin{array}{l}40-\mathrm{ft}-1 \text { ong by } 8-10-\mathrm{ft} \text {-deep by } 15-\mathrm{ft} \text {-wide area } \\
\text { within } 10 \mathrm{ft} \text { of the north wall of the } 207-\mathrm{U} \\
\text { retention basin, constructed to contain } \\
\text { sludge from the bottom of the northern } \\
\text { section of } 207-\mathrm{U} \text { retention basin at an } \\
\text { unknown time. The sludge was originally } \\
\text { covered with } 4 \mathrm{ft} \text { of clean soil, but has been } \\
\text { recontaminated and is presently a Surface } \\
\text { Contamination Area. }\end{array}$ \\
\hline
\end{tabular}




\begin{tabular}{|c|c|c|}
\hline SITE & UPR NUMBER & SITE DESCRIPTION/LOCATION \\
\hline UN-216-W-23 & UPR-200-W-113 & $\begin{array}{l}\text { Area near the } 241-T X-155 \text { diversion box, } \\
\text { contaminated by rabbit feces in } 1977 \text {. } \\
\text { Surveys of the area to determine the } \\
\text { contamination source revealed low-level } \\
\text { beta/gamma contamination, below and to the } \\
\text { west of the } 241-T X-155 \text { diversion box. } \\
\text { Radiation levels increased during attempted } \\
\text { excavation of the contamination causing } \\
\text { assumptions that a nearby waste transfer line } \\
\text { was the underground source of contamination. } \\
\text { The area was covered with clean soil and } \\
\text { revegetated in } 1990 \text {. }\end{array}$ \\
\hline UN-216-W-24 & UPR-200-W-114 & $\begin{array}{l}\text { Radioactive particulate matter resulting from } \\
\text { operations activities in the } 241-S X \text { tank } \\
\text { farm, the } 241-S X-151 \text { diversion, and the } \\
241-S-151 \text { diversion box spread over the } \\
\text { ground surface of the subject area during the } \\
\text { many years of operations. A number of } \\
\text { cleanup campaigns reduced the amount of } \\
\text { contamination, but a residue of the original } \\
\text { remains on the ground. }\end{array}$ \\
\hline$U N-216-W-25$ & UPR-200-W-115 & $\begin{array}{l}\text { This site consists of a surface contamination } \\
\text { zone associated with clean-out boxes on the } \\
\text { feed line. Area posted as Surface } \\
\text { Contamination. }\end{array}$ \\
\hline$U N-216-W-26$ & UPR-200-W-116 & $\begin{array}{l}\text { This area became contaminated with } \\
\text { particulate matter spread by wind from the } \\
204-5 \text { waste storage tank exhaust and the } \\
\text { related railroad tanker waste unloading } \\
\text { station. The site is located } 300 \text { feet north } \\
\text { of the } 202-5 \text { building, next to the REDOX } \\
\text { railroad cut. Area was interim stabilized in } \\
1993 \text {. }\end{array}$ \\
\hline$U N-216-W-27$ & UPR-200-W-117 & $\begin{array}{l}\text { This site results from contaminated liquid } \\
\text { and particulate matter dropping from railroad } \\
\text { cars servicing the } 221-U \text { facility during the } \\
\text { Uranium Recovery and Equipment } \\
\text { Decontamination Programs, and the various } \\
\text { storage and unloading activities. These } \\
\text { activities started in } 1952 \text { with the Uranium } \\
\text { Recovery Program (1952-1957) and have } \\
\text { continued intermittently to the present. The } \\
\text { site was designated as a radiation zone, but } \\
\text { has since been released as contamination has } \\
\text { decayed to background levels. }\end{array}$ \\
\hline
\end{tabular}


WHC-SP-0098-6

\begin{tabular}{|c|c|c|}
\hline SITE & UPR NUMBER & SITE DESCRIPTION/LOCATION \\
\hline$U N-216-W-28$ & UPR-200-W-118 & $\begin{array}{l}\text { This site is the result of drippings and } \\
\text { spills from the reclaimed nitric acid } \\
\text { unloading stations in the } 211-U \text { chemical tank } \\
\text { farm. Wind-borne particulate matter spread } \\
\text { to the ground surface outside the concrete } \\
\text { unloading station. This site was designated } \\
\text { a radiation zone, but has since been released } \\
\text { as contamination has decayed to background } \\
\text { levels. }\end{array}$ \\
\hline UN-216-W-29 & UPR-200-W-164 & $\begin{array}{l}\text { The site consists of spotty surface } \\
\text { contamination beneath the steam line from the } \\
204-S \text { storage tanks to the } 224-U \text { building. } \\
\text { In } 1986 \text { the zone was reduced to a small area } \\
\text { of spotty surface contamination beneath the } \\
\text { steam line. Interim stabilized in } 1993 \text {. }\end{array}$ \\
\hline$U N-216-W-30$ & UPR-200-W-165 & $\begin{array}{l}\text { The site consists of speck surface } \\
\text { contamination measuring } 200 \mathrm{cpm} \text { to } 45 \mathrm{mr} / \mathrm{hr} \text {, } \\
\text { and the sites location is northeast of } \\
241-S / S X / S Y \text { tank farms. }\end{array}$ \\
\hline$U N-216-W-31$ & UPR-200-W-166 & $\begin{array}{l}\text { The site contains spotty contamination which } \\
\text { is suspected to have originated from } 241-T \\
\text { tank farm. In } 1991 \text {, the interior fenced area } \\
\text { of } 241-T \text { tank farm was decontaminated, and a } \\
\text { layer of clean gravel was placed over the } \\
\text { area. In } 1991 \text { and } 1992 \text { radiologically } \\
\text { contaminated soil was scraped and } \\
\text { consolidated along the hillside on the west } \\
\text { side of } 216-T-14 \text { through } 216-T-17 \text { units. A } \\
\text { layer of clean soil was placed over the } \\
\text { consolidated contaminated soil area. }\end{array}$ \\
\hline$U N-216-W-32$ & UPR-200-W-167 & $\begin{array}{l}\text { Migration of contamination from TY tank farm } \\
\text { over time. The site location is northeast of } \\
\text { the } 241-T \text { tank farm west of } 216-T-14 \text { through } \\
17 \text { trenches; it surrounds the } 207-T \text { retention } \\
\text { basin. The contaminated soils were scraped } \\
\text { and disposed in the } 200 \text { West burial grounds. } \\
\text { Clean gravel was placed over the excavated } \\
\text { surface. }\end{array}$ \\
\hline UN-216-W-33 & UPR-200-W-163 & $\begin{array}{l}\text { The site contains radiologicaliy contaminated } \\
\text { vegetation growing above the underground } \\
\text { pipeline. The contamination came from an } \\
\text { underground pipe line from } 224-T \text { (UO3) to the } \\
216-U-8 \text { crib. Area interim stabilized in } \\
1994 \text {. Pipeline was reposted as URM rest of } \\
\text { area was released from posting status. }\end{array}$ \\
\hline$U N-216-W-34$ & $N / A$ & $\begin{array}{l}\text { Waste water sump inside the } 2724-W \text { laundry } \\
\text { facility. }\end{array}$ \\
\hline
\end{tabular}


WHC-SP-0098-6

\begin{tabular}{|c|c|l||}
\hline SITE & UPR NUMBER & \multicolumn{1}{|c|}{ SITE DESCRIPTION/LOCATION } \\
\hline UN-216-W-35 & UPR-200-W-161 & $\begin{array}{l}\text { 50 ft east of the 241-U tank farm and } 100 \mathrm{ft} \\
\text { north of the 207-U retention basin. }\end{array}$ \\
\hline UN-216-W-36 & $\begin{array}{c}\text { UPR-200-W- } \\
160,\end{array}$ & \\
\hline UN-216-W-37 & UPR-200-W-162 & $\begin{array}{l}\text { The area adjacent to and east of the 221-U } \\
\text { building; (UN-216-W-9 and 216-W-11 are } \\
\text { located within this larger unplanned release } \\
\text { site). The site consists of surface speck } \\
\text { contamination This site al so encompasses } \\
\text { previous spili areas UPR-200-W-101 and } \\
\text { UPR-200-W-138. Some contamination was } \\
\text { removed using buckets and shovels by Tank } \\
\text { Farm Operations in March and October 1991. }\end{array}$ \\
\hline
\end{tabular}

$\mathrm{cpm}=$ counts per minute

$\mathrm{dpm}=$ disintegrations per minute

$U R M=$ underground radioactive material 
WHC-SP-0098-6

This page intentionally left blank. 
WHC-SP-0098-6

\section{DISTRIBUTION}

\section{Number of Copies}

\section{ONSITE}

3 U.S. Department of Energy, Richland Operations Office
M. J. Furman
R3-80
J. B. Hall
A5-55
D. C. Ward
A5-55

4 Pacific Northwest Laboratory
R. L. Dirkes $(4)$
K6-61

10 Bechtel Hanford Incorporated
R. A. Lewis
H4-86
L. A. Mihalik
H4-79
W. L. Osborne
T3-11
K. A. Smith
NI-06
C. D. Wade (6)
$\times 0-41$

41 Westinghouse Hanford Company
H. A. Besel
T1-27
L. P. Diediker
$\mathrm{T} 1-30$
J. J. Dorian
$\mathrm{H} 6-20$
J. E. Hodgson
$X 7-02$
A. R. Johnson
H6-2O
B. M. Markes
$\mathrm{H} 6-20$
S. M. Mckinney
TI -30
D. L. Mitchell
$\mathrm{H} 6-32$
J. E. Parsons
C. J. Perkins (6)
$\mathrm{N} 1-06$
M. D. Rowe (6)
$\mathrm{XO}-21$
J. W. Schmidt (10)
$\mathrm{T} 1-25$
$\mathrm{H} 6-2 \mathrm{O}$
J. A. Seamans
N2-04
R. R. Thompson
H6-32
D. J. Watson
$\mathrm{X} 0-41$
R. L. Watts
L6-52
Central Files
L8-04
Document Processing and Distribution (2) EDMC
L8-15
H6-08
Information Release
Administration
H4-17
OSTI
ᄂ8-07 
WHC-SP-0098-6

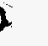

This page intentionally left blank. 